\title{
Nanoscale Waveguiding Studied by Lensless Coherent Diffractive Imaging using EUV High-Harmonic Generation Source
}

\author{
Dissertation \\ zur Erlangung des mathematisch-naturwissenschaftlichen Doktorgrades \\ "Doctor rerum naturalium" \\ der Georg-August-Universität Göttingen \\ im Promotionsprogramm ProPhys \\ der Georg-August University School of Science (GAUSS)
}

vorgelegt von

Herrn Dipl.-Ing. Sergey Zayko

aus USSR

Göttingen, 2016 
Betreuungsausschuss

Prof. Dr. Claus Ropers, IV. Physikalisches Institut

Prof. Dr. Tim Salditt, Institut für Röntgenphysik

$\underline{\text { Mitglieder der Prüfungskommission }}$

Referent: $\quad$ Prof. Dr. Claus Ropers, 4. Physikalisches Institut

Korreferent: $\quad$ Prof. Dr. Tim Salditt, Institut für Röntgenphysik

Weitere Mitglieder der Prüfungskommission

Prof. Dr. Hans-Ulrich Krebs, Institut für Materialphysik

Prof. Dr. Astrid Pundt, Institut für Materialphysik

Prof. Dr. Ansgar Reiners, Institut für Astrophysik

Prof. Dr. Michael Seibt, 4. Physikalisches Institut

Tag der mündlichen Prüfung: 21. 09. 2016 


\section{Abstract}

The challenge of nanometric imaging drives intense efforts in applied sciences and fundamental research. Following the physical law of diffraction, the utilization of extreme ultraviolet (EUV) or X- ray radiation for imaging extends the ultimate resolution limit given by the illumination wavelength down to nanoscale dimensions. However, despite the great potential, the resolution is governed by imperfections inherent to optical elements employed. In this cumulative thesis, a table-top source of EUV radiation based on high harmonic generation (HHG), is developed and applied for lensless coherent diffractive imaging (CDI). Real-space images of microscopic objects are obtained solely on the basis of their far-field diffraction patterns, eliminating the need of any optical element and therefore any aberrations they may have. Benefiting from the exceptional coherence of the source developed, a diffraction-limited imaging is demonstrated with a spatial resolution of illuminating wavelength down to $30 \mathrm{~nm}$.

The microscopic images obtained via CDI allow for a detailed study of electric field distributions on the exit surface of the investigated structures. The experiments reveal that electric fields emerging from metallic structures are strongly affected by the polarization of the illuminating probe as well as propagation effects within the sample, indicating the limitations of the projection approximation. The above observations are attributed to waveguiding phenomena, thus, demonstrating extreme ultraviolet waveguiding in nanoscale slab structures. By direct imaging of the spatial distribution of the underlying electromagnetic modes exiting the sample, a strong polarization contrast of metallic waveguides with high aspect-ratio is experimentally identified. The guiding and polarizationsensitiveness are explored through an analytical approach based on the expansion in eigenmodes as well as numerical simulations to corroborate the experimental findings.

Additionally, a novel approach to analyze and control the polarization state of EUV radiation by means of single image acquisition is developed. Such an analyzer, employing a set of nanoscale slab waveguides provides for an advanced characterization scheme for the polarization state of high harmonics.

These results on CDI beyond the projection approximation using high harmonics demonstrate the potential of such a source for imaging, and pave the way towards applications in material science, for example, the spatial investigation of chiral phenomena, such as magnetism, enabling nanoscale magnetic imaging with a compact tabletop setup. Furthermore, HHG enables to date shortest possible pulse durations offering the opportunity 
to study ultrafast phenomena with unprecedented temporal resolution. 


\section{Contents}

List of Figures vii

1 Introduction 1

1.1 Nanoscale imaging . . . . . . . . . . . . . . . 7

1.2 Coherent Diffractive Imaging . . . . . . . . . . . . . . . . . 9

1.3 Probes for lensless imaging . . . . . . . . . . . . . . . 17

1.4 High-harmonic generation . . . . . . . . . . . . . . . 17

1.4.1 Three-step model . . . . . . . . . . . . . . . . . . 20

1.4.2 Phase matching . . . . . . . . . . . . . 22

1.4.3 Lensless imaging with high-harmonic radiation . . . . . . . . . 24

2 Toroidal grating astigmatism of high-harmonics characterized by EUV Hartmann sensor

3 Coherent diffractive imaging beyond the projection approximation: Waveguiding at extreme ultraviolet wavelengths

4 Polarization contrast of nanoscale waveguides in high harmonic imaging 49

5 General discussion $\quad 59$

5.1 High-resolution coherent diffractive imaging . . . . . . . . . . . . . 59

5.2 Lensless imaging of the guided modes in the EUV spectral range . . . . . 68

5.3 Polarization contrast in nanoscale structures . . . . . . . . . . . . . . . . 69

5.4 Capabilities and limitations of the developed source for lensless imaging . 74

5.5 Chapter summary . . . . . . . . . . . . . . . . . . 76

5.6 Future prospects . . . . . . . . . . . . . . . . . 78

$\begin{array}{ll}\text { A Appendix } & 89\end{array}$ 
A.1 Experimental setup and preparations for operation . . . . . . . . . . 89

A.2 Optical alignment . . . . . . . . . . . . . . . . . . . . . . . . . 94

A.3 High harmonic generation optimization and characterization . . . . . 96

$\begin{array}{ll}\text { Bibliography } & 99\end{array}$

$\begin{array}{ll}\text { Author contribution } & 127\end{array}$

$\begin{array}{lr}\text { Publications and Conferences } & 129\end{array}$

$\begin{array}{ll}\text { Acknowledgments } & 131\end{array}$ 


\section{List of Figures}

1.1 Dynamics of crystalline structures exposed to intense X-ray pulses . . . . 4

1.2 Schematic representation of phase retrieval algorithms . . . . . . . . 11

1.3 State of the art lensless imaging capabilities . . . . . . . . . . . . . . 14

1.4 Schematic of lensless imaging experiments . . . . . . . . . . . . . 15

1.5 Schematic representation of the three step model in high-harmonic generation . . . . . . . . . . . . . . . . . 18

1.6 High-harmonic radiation with keV photon energy . . . . . . . . . . . . . 19

1.7 Possible ionization mechanisms _. . . . . . . . . . . . . . 20

1.8 First experimental demonstration of coherent diffractive imaging using high harmonics ....................... 25

2.1 Setup of HHG source with a single toroidal grating . . . . . . . . . . . 29

2.2 Wavefront of the $25^{\text {th }}$ harmonic measured at different angles of incidence $\quad 30$

2.3 Astigmatic waist difference as a function of the angle of incidence . . . . 32

3.1 Schematic of the experimental setup . . . . . . . . . . . . 36

3.2 Diffraction patterns and reconstructions of the test structures . . . . . . 38

3.3 Finite element modeling of the wave propagation confined by the optically dense media . . . . . . . . . . . . . . . . . . . . 39

3.4 Semi-analytical solution of the modal expansion approach for excited modes within the investigated structures . . . . . . . . . . . . . . 44 41

3.5 Sketch of the sample structure and back side SEM image . . . . . . . . . 42

3.6 Comparison of the imaging quality for short and long exposure time ex-

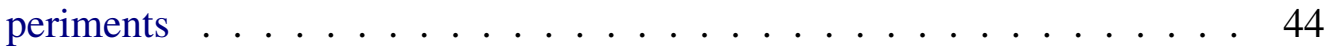

3.7 Estimation of the achieved resolution by means of phase retrieval transfer function . . . . . . . . . . . . . . . . . 45

3.8 Examining Fourier truncations $\ldots \ldots \ldots$. . . . . . . . . . 47 
4.1 Experimental setup for lensless imaging using HHG source . . . . . . . . 51

4.2 Demonstration of polarization contrast in CDI . . . . . . . . . . . . 52

4.3 Wavelength-dependence of polarization contrast . . . . . . . . . 53

4.4 High polarization contrast of nanoscale waveguides . . . . . . . . . . 55

5.1 Spherical corrections of diffraction data with high numerical aperture . . 62

5.2 Coherent diffractive imaging using different illuminating wavelengths . . 63

5.3 Dependence of the spatial resolution on the distance between the sample and detector . . . . . . . . . . . . . . . . . 665

5.4 Resolution estimate with the phase retrieval transfer function showing the highest spatial frequencies that are consistently reconstructed . . . . . . 66

5.5 Polarization contrast in nanoscale slab waveguides $\ldots \ldots$. . . . . 70

5.6 Deconvolution of the autocorrelation support . . . . . . . . . . . 73

5.7 Spectrum of high harmonics generated in neon . . . . . . . . . . 75

5.8 Capabilities of lensless imaging technique . . . . . . . . . . . 76

5.9 Concept of efficient frequency doubling . . . . . . . . . . . . 80

5.10 Generation scheme for circularly polarized harmonics . . . . . . . . . . 83

5.11 High-harmonic spectra generated in argon with a bichromatic driver at a fixed capillary pressure . . . . . . . . . . . . . 85

5.12 Spectra of circularly polarized harmonics . . . . . . . . . . 86

5.13 Compact in-line generation scheme for circularly polarized harmonics . . 87

5.14 Spectrum of circularly polarized harmonics for XMCD measurements . . 88

A.1 Laser-induced ablation of the capillary walls . . . . . . . . . . . . . . 92

A.2 Experimental setup for imaging experiments . . . . . . . . . . . . . 93

A.3 Pressure dependent phase matching of HHG at fixed laser intensity. . . . 97 


\section{Chapter 1}

\section{Introduction}

Discovering and understanding nature becomes possible if the resolving power of a measuring device is sufficient to observe the investigated phenomena. In the case of optical imaging, the maximum angular resolution or the so-called visual acuity of a human eye is 1/60 degree, limiting the size of the smallest resolvable features to about 30 micrometers [1]. However, a variety of far-reaching contributions to the wide range of technological and scientific progress is directly attributed to developments at scales far beyond this limit. The most prominent examples being the rapid evolution of micro- and nanoelectronics, and cellular biology. The field of microscopy extends the capabilities of a human eye by providing a magnified image of the investigated object using a variety of contrast forming mechanisms. Invented in the late 16th century and thereafter developed by Robert Hooke, optical microscopes revealed "new worlds" and most remarkably the world of a living cell. Over the following centuries, microscopes continuously evolved, gaining a steadily increasing importance in fundamental research as well as medical and industrial applications.

Similar to the very first imaging devices, current state-of-the-art optical microscopy employs visible light to probe specimens. Such microscopes allow for the imaging of fabricated samples and living matter in vivo. In a typical design, the light used as a probe is transmitted through or reflected from a sample and passes the lens to form a magnified image. In this case, the image contrast arises from local variations of the absorption along the light's optical path, as well as, the morphology of the sample. Due to its ease of use, compactness and reasonable performance, optical microscopy has become a widespread technique for studying microscopic samples. To investigate structures exhibiting low contrast, different illumination techniques as well as contrast mechanisms were developed. 
For example, in dark-field microscopy and its variation ultramicroscopy, samples are illuminated at very large angles, so that only the scattered light reaches the objective lens and forms the image. In this case, the structures investigated appear as luminous points on a dark background, and the image contrast is significantly improved. To investigate transparent samples, a phase contrast microscope can be used. Here, a phase shift introduced to the probe by local variations of the refractive index or thickness inhomogeneities in the sample is detected. Eventually, advances in microscopy designs, technological developments of manufacturing processes, and utilization of new materials contributed to a remarkable improvement of the capabilities of modern microscopes.

Across the wide applicability and the great diversity of microscopes, one of the most important characteristics for all imaging techniques is the resolution, i.e., the smallest feature size that can be resolved in the magnified image. Back in 1873, the German physicist Ernst Abbe discovered that the resolving power of any imaging system is fundamentally limited by the diffraction of waves, and approximated the diffraction-limited half pitch resolution as:

$$
R_{h p} \approx \lambda / 2 n \sin \theta
$$

Here, $\lambda$ is the wavelength of the illuminating radiation in vacuum, $n$ is the refractive index of the medium between the lens and the specimen, and $n \sin \theta$ is the numerical aperture $(N A)$ of the imaging system. This expression was later specified by Lord Rayleigh as a measure for the separation between two Airy discs in order to be distinguished:

$$
R_{h p}=0.61 \lambda / n \sin \theta \text {. }
$$

Special objective lenses with small focal lengths and large angular apertures combined with the use of immersion oils or glycerol with high refractive index enable imaging at numerical apertures as high as 1.5 [2]. Employing green light illumination and an imaging lens of a perfect quality, the smallest feature size distinguishable by an optical microscope is approximately $200 \mathrm{~nm}$. Equations 1.1 and 1.2 show that a great improvement in resolution can be achieved by using shorter wavelengths for illumination, e.g., extreme ultraviolet (EUV) radiation or X-rays. Alternatively, a well-defined beam of high energy particles, such as electrons or ions, can be used as a probe. In this case, the resolution limit determined by diffraction is associated with the de Broglie wavelength of matter [2]. Depending on the energy of the probe particle, the associated de Broglie wavelength can 
be as small as few picometers. However, the manufacturing of aberration-free lenses for such probes is particularly challenging. The same applies to lenses for EUV radiation and X-rays, i.e., at wavelengths below 100-150 nm. Hence, the experimentally achievable resolution of microscopes based on X-rays or high energy particles is very far from the corresponding fundamental limits, and is mainly governed by the aberrations introduced due to imperfections of the image forming elements. Therefore, the development of new concepts to overcome this hurdle is of great importance.

Pushing the limits of conventional microscopes and the development of new methods have led to the evolution and diversification of modern microscopy into several branches of powerful imaging techniques employing a variety of different contrast mechanisms, image forming methods and probes. One of the most promising techniques that can close the gap between the achievable resolution and fundamental frontiers is coherent diffractive imaging (CDI), where image-forming elements such as lenses or focusing mirrors are eliminated [3]. Instead, a computer algorithm is used to reconstruct the real space image from its far-field diffraction pattern under illumination with a coherent probe [4]. In principle, such a scheme enables imaging with a spatial resolution solely determined by diffraction (cf. Eq. 1.1), thereby greatly improving the imaging capabilities. Moreover, the high penetration of X-rays compared to visible light or particle beams is beneficial for thick or buried samples. Further details on lensless imaging techniques are given in Section 1.2.

In many applications, however, the key properties of the investigated specimen are concealed in its temporal evolution, e.g., ultrafast structural and electronic dynamics. Similarly, for radiation-sensitive samples, the limiting factor can be the total flux or radiation dose obtained during the exposure [5] and, therefore, the data should be collected before the sample is subjected to significant changes [6,7]. A very illustrative example is the simulation performed by Barty et al. [8] shown in Fig. 1.1. The authors model the temporal evolution of lysergic acid diethylamide, commonly known as LSD, after illumination with an intense X-ray pulse. The presented results demonstrate the significance of radiation-induced damage, resulting in a full destruction of the crystalline order by an intense X-ray pulse after an exposure of only 100 femtoseconds. Nevertheless, the diffraction data can be recorded within this time interval, if the probe pulse is sufficiently short [6]. Similarly to a crystalline structure, a single molecule, such as a protein, can be completely disintegrated within just a few tens of femtoseconds after the illumination with ultrashort X-ray pulses [9], highlighting the demand for ultrafast probes [7, 10, 11]. 

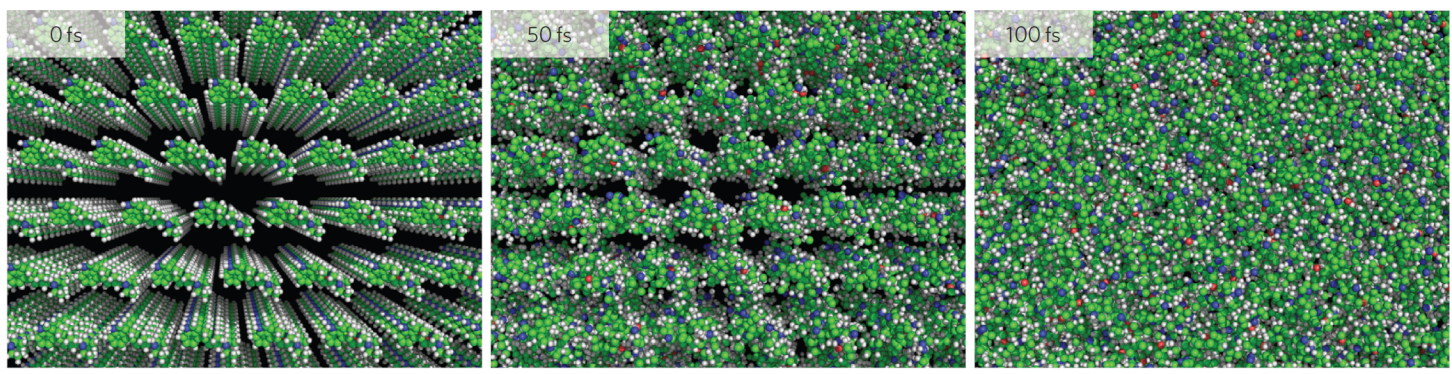

Figure 1.1: Modeling of the dynamics of a crystalline structure exposed to an intense X-ray pulse. Reprinted by permission from Macmillan Publishers Ltd: Nature Photonics (Ref. [8]), copyright (2012).

Combining both fundamental properties - short wavelengths and ultrafast pulse duration - revolutionary light sources based on the principle of high harmonic generation (HHG) [12] and X-ray free electron lasers (FEL) carry great potential for imaging ultrafast phenomena with nanometer resolution $[6,13]$.

Free electron lasers generate ultrashort pulses of tunable photon energy with enormous brightness, but due to the large dimensions (up to several kilometers) of FEL setups and their high costs, only a few facilities in the world are available. In contrast, novel HHG sources provide coherent short-wavelength radiation with a broad spectral range on a table-top scheme, albeit at lower photon energies. Due to the compactness of the setup, availability, and excellent properties, HHG is of great interest for a variety of applications in atomic and molecular physics $[14,15,16]$ as well as surface and materials science $[17,18]$. Furthermore, HHG gave rise to sub-femtosecond light pulses, introducing the new field of attosecond science [19, 20, 21, 22]. Having undergone a rapid development during the last two decades, HHG sources, utilizing different generation schemes, became indispensable scientific tools due to their exceptional properties. This includes the available spectral range - from a few electron volts to $\mathrm{keV}$ [23], the high spatial coherence $[24,25,26]$, polarization control $[27,28,29,30]$ and pulse durations down to sub-100 attoseconds [31]. Remarkably, the generated EUV photon flux of compact HHG sources, including the one presented in this thesis, exceeds $10^{10}$ photons/second, which is already comparable to the EUV flux of a small synchrotron facility ${ }^{1}$. Moreover, the use of innovative fiber lasers demonstrates further improvement of the generated EUV flux, up to $10^{13}$ photons/second at $30 \mathrm{eV}$ photon energy, owing to the increased repetition rates

\footnotetext{
${ }^{1}$ Available narrow-band EUV photon flux at BESSY II - at present, the only third generation synchrotron in Germany - is between $10^{9}$ and $10^{13}$, depending on the beamline.
} 
and average power [32]. Finally, using advanced generation geometries, it is possible to control the polarization of the emitted harmonics. Although a high-harmonic generation scheme for an effective generation of circularly polarized harmonics has been proposed and experimentally demonstrated almost 20 years ago [33], the polarization state of the emitted harmonics was not confirmed until recently [27, 34]. Further developments allowed for the extension of the available photon energies up to $160 \mathrm{eV}$ [35]. Such schemes extend the capabilities of high harmonic radiation to studies involving the investigation of chiral properties, e.g., magnetic circular dichroism.

In this work, a compact HHG source of coherent EUV radiation, operating in combination with a toroidal diffraction grating monochromator and suitable for high-resolution lensless imaging as well as ultrafast spectroscopy, is presented. The key properties of the employed light source are characterized and optimized for coherent diffraction imaging, enabling the investigation of nanoscale objects with diffraction-limited resolution. The properties of nanoscale slab waveguides are studied at the EUV spectral range by direct imaging of the propagating modes at the exit surface of the objects under investigation. This cumulative thesis is based on three original publications that constitute Chapters 2 4 , incorporating the most important results of the conducted experiments.

\section{Outline}

The thesis is organized as follows: Chapter 2 describes the characterization and subsequent optimization of the generated EUV radiation using caustic measurements and wave front characterizations with a Hartmann-Shack-sensor. Furthermore, a theoretical description of the underlying processes is provided in the form of an optical matrix approach.

In Chapter 3, the implementation of the developed source for lensless coherent imaging is presented. The complex transmission function of microscopic objects at a wavelength of $35 \mathrm{~nm}$ is reconstructed solely on the basis of far-field diffraction patterns. Diffractionlimited resolution of the obtained images is demonstrated, indicating almost perfect convergence of the phase retrieval algorithm used. The high spatial resolution of the obtained images allows for a detailed inspection of the reconstructed exit waves, revealing their strong modulation at the exit surface of the sample. This modulation is associated with multiple scattering of the probe within the object, caused by the strong spatial confinement of the propagating light within optically thick materials, which induces wave- 
guiding effects. A finite element model as well as an analytical solution of the observed phenomenona is provided and compared with the experimental data. Additionally, these results demonstrate the potential of such structures for EUV polarization and mode filtering.

Chapter 4 describes the development of advanced optical devices based on nanoscale slab waveguides for polarization control of EUV radiation. Moreover, a polarization analyzer is designed and utilized for the characterization of the polarization state of EUV radiation within a single acquisition. Unlike conventional reflection-based EUV polarizers, the analyzer developed allows for an unambiguous distinction between light with circular polarization and unpolarized radiation, owing to the inherent phase shift of the employed diffraction grating. Furthermore, these results demonstrate for the first time that CDI with high harmonics can be effectively used to study polarization sensitive phenomena.

Chapter 5 summarizes the outcome of the studies and provides a general discussion of the results obtained as well as an outlook regarding possible implementations in applied science and industry.

The following section provide a concise overview of the methodological aspects used in this work and introduce the required background knowledge for deeper insights into the subject. 


\subsection{Nanoscale imaging}

Increasing interest in the investigation of a variety of samples not suitable for conventional optical microscopy in combination with the demand for improving resolution and contrast stimulated versatile developments and evolution of imaging methods and tools. Most modern imaging techniques can be classified according to two main aspects: the imageforming mechanism and the probe that interacts with the sample. The image formation can involve optical elements or lensless techniques described in Chapter 1.2. At the same time, depending on the acquisition procedure, measurements can be done using a full-field exposure (illumination of a large area), allowing for a single acquisition measurements, or in a scanning mode, where the probe interacts with the object only at a small well defined area and the overall image is recovered from the information at each individual scan step.

Historically, visible light became the prevalent probe for imaging due to the availability of light sources and suitable materials for image forming elements, and the natural sensitivity of the human eye to this part of the spectrum. First microscopes were able to obtain magnified images using variations in absorption or reflection coefficients within the object to form image contrast. To increase the capabilities of conventional optical microscopes, tremendous effort has been invested into inventing and developing new illumination and detection schemes. Significant contributions have been awarded by Nobel Prizes in 1925, 1953 and 2014 for the invention and associated works on ultramicroscopy, phase contrast microscopy and super-resolution fluorescent microscopy, respectively.

Advances in technologies for manipulation of charged particles, i.e., magnetic and electrostatic lenses, enabled imaging using focused electron or ion beams as a probe with the de Broglie wavelength as short as few picometers. This led to a significant improvement in resolving capabilities and provided new degrees of freedom in terms of contrast forming schemes enabling the investigation of various properties of matter, e.g., structural, magnetic, electronic properties, etc. The development of electron optics and design of the first electron microscope has been recognized by the Nobel Prize in 1986. Similar to electrons, beams of high energy particles can be used. Another type of wave probe that became particularly useful in medicine applications are high frequency acoustic waves "ultrasound".

Locally defined interactions with the sample improve spatial resolution of the image to approximately the size of the probe dimension. When a very sharp tip is positioned in the vicinity of the sample surface, the forces from the surface to the tip are measured. If such 
measurements are done over some finite area with small scanning steps, the topology of the surface can be detected with a very high precision. A set of microscopy techniques is based on this principle. Depending on the sample and the tip used, different forces can be detected, including van der Waals forces, magnetic and electrostatic forces, etc. Here, the image is formed according to the information after "palpation" of the sample with the probe tip at known positions and the distribution of the force investigated at the sample surface can be mapped. This technique is known as atomic force microscopy (AFM). Similarly, a tunnel current resulting from an applied bias voltage between a conductive sample and a metal tip can give a precise measurement of the distance between the sample and the tip at the known position. For the design of a scanning tunneling microscope (STM) a Nobel Prize in physics was shared in 1986 with the inventor of the electron microscope. Interestingly, a scanning scheme with a well-defined probe of visible radiation, for example, produced by a very thin optical fiber, can significantly improve the resolution of the imaging system using the visible light and even break the diffraction limit given in Eq. 1.1 by exploiting properties of evanescent waves [36, 37].

Depending on the properties of the sample investigated, different probes have their advantages and limitations. Atomic force microscopes as well as STMs provide atomic resolution, but - due to the scanning principle - these techniques are slow, limited to a small area of the surface, require low roughness and are very sensitive to vibrations. Electron microscopy is superior in terms of spatial resolution and can provide sub-Ångstrom imaging [38], but it also has a number of limitations. It is not applicable at ambient pressures and, due to a small penetration depth of the electron beam, it is limited to relatively thin samples of a few tens of nanometers in thickness or a sample's surface. Furthermore, charging effects can be a serious issue for non-conductive specimens.

In contrast, X-ray microscopy is not affected by these constraints. However, for Xrays the absence of efficient optical elements hinders an effective utilization of refractive optics, such as lens systems that employ refraction at surfaces between materials with different refractive indices. In the X-ray spectral range, the real part of the refractive index is very close to unity ${ }^{2}$, therefore the imaging system must involve an assembly of hundreds of lenses, often called compound refractive lenses (CRLs) [39]. Although a successful implementation of CRLs for imaging has been demonstrated even with hard X-rays, the spatial resolution obtained barely reaches a few hundreds of nanometers [40, 41]. In

\footnotetext{
${ }^{2}$ To classify the real part of the refractive index $(n)$ in the X-ray spectral range, the so-called index decrement $\delta$ is used, where $\delta=1-n$. Typical magnitudes of the index decrement are below $10^{-4}$.
} 
contrast to X-rays, extreme ultraviolet radiation experiences a very high absorption in most materials, diminishing the ability to build an effective refraction lens. An alternative way of focusing X-rays or EUV radiation is the utilization of Fresnel zone plates, where diffraction instead of refraction is used [2]. Such a scheme has an improved focusing ability. However, low efficiency and still rather poor focusing, limited by the manufacturing process, reduce the resolution capabilities of an imaging system employing a focusing zone plate. Using optical elements, X-ray microscopy rarely provides imaging with a resolution below $20 \mathrm{~nm}[42,43,44,45]$, which is very far from the theoretical limit given by the illumination wavelengths (cf. Eq. 1.1).

In summary, the utilization of lensless imaging techniques has a significant advantage over conventional lens-based microscopy in the EUV and X-ray spectral ranges and may allow for an exploration the full potential of X-rays for imaging [46].

\subsection{Coherent Diffractive Imaging}

When a non-periodic sample is illuminated with a coherent beam, the electric field distribution in the far-field diffraction pattern is proportional to the Fourier transform of the electric field distribution at the exit surface of the sample, which is valid within the Fraunhofer approximation of the diffracted light. Knowledge of the intensity and phase distributions in the far-field allows for a direct reconstruction of the object's transmission function in the case of a uniform illumination. However, only the electric field intensity can be measured experimentally, whereas the phase information is lost. Since the phase is not unique, i.e., a variety of data sets can lead to very similar amplitudes in the farfield, direct reconstruction of the sample from it's far-field diffraction is not possible. The recovery of the missing information is commonly known as the phase problem and is a major part of crystallography. The periodic structure of the crystal lattice significantly simplifies the solution of this problem, since X-rays incident onto the crystalline sample will diffract into specific directions - discrete Bragg peaks. The information on angles and intensities of these diffraction spots is sufficient for a full crystal structure determination [47]. Thus, since the discovery of X-ray diffraction by crystals [48], crystallography has become one of the central multidisciplinary sciences and has led to a number of scientific achievements that were awarded by the Nobel Prize more than 20 times by now.

In contrast to crystalline specimens, the diffraction pattern of a non-periodic sample is continuous, rather than consisting of well-defined Bragg peaks, and generally the struc- 
tural information is not available without the phase information. The first algorithm for solving the phase problem, i.e., retrieving the phase of the scattered signal from the measured far-field intensities for non-periodic samples, was proposed by Gerchberg and Saxton in 1971 [49] and later developed by Fienup [3, 4]. The main requirements for a successful phase recovery of a non-crystalline sample are the spatial isolation of the object (or illumination of the object with a well confined probe) and recording the scattered intensities with sufficient resolution (oversampling). The information on the object's size is encoded in the far-field intensities and can be obtained directly from the autocorrelation of the object, given by the inverse Fourier transform of the measured diffraction intensities. Sampling the diffraction pattern with a resolution at least twice finer than the Nyquist frequency (inverse size of the object) is necessary, since the autocorrelation is twice larger than the object itself in each dimension. This condition also implies a constraint on the size of the object or on a field of view in case of a confined probe. The information required for a reconstruction is the illuminating wavelength and the distance between the sample and detector. Any additional information about the object, or so-called a priori knowledge, can be used as a constraint to narrow down the set of possible solutions and simplify the reconstruction procedure. In other words, the phase retrieval of non-periodic samples is a technique for finding thew set of phases that satisfy certain constraints and correspond to the measured far-field intensities. The development of lensless imaging techniques has evolved into a separate microscopy branch, currently known as coherent diffractive imaging (CDI).

Phase retrieval Typically, a phase retrieval algorithm starts with a first guess, e.g., a random phase distribution that is added to the measured far-field amplitudes (square roots of measured intensities). The second step is an inverse Fourier transform of the measured amplitudes with the added phases. This step provides a guess on the electric field distribution at the object in real space, which corresponds to the measured diffraction data. A fast Fourier transform (FFT) algorithm is used to compute the discrete Fourier transform numerically. In real space, the constraints obtained from a priori knowledge are introduced. For example, in the case of non-complex objects, the relative phase over the entire object can be set to zero which simplifies the reconstruction process. However, amplitudes of the variables are still to be reconstructed. The number of variables is the total number of the real space pixels which is equal to the number of pixels of the diffraction pattern measured by the CCD camera in the far-field. The information about the object is now 
used to create the real space support, i.e., to define variables (pixels) with known values. These values are provided by the known scattering density of the object. In the simplest case, these are zero-valued pixels, for example, pixels in the area covered with an opaque mask or when the object is a transmission mask itself. Now, the current set of variables has to be modified to satisfy the real space constraint, giving a projection of the current guess for the solution onto the real space constraint. When the real space constraint is satisfied, another FFT is performed to convert the updated real space solution guess to the frequency domain, where absolute values of the variables are replaced with the experimental data to fulfill the so-called modulus constraint. This is the final step of the first run of a reconstruction loop and the algorithm is further repeated from step two. Thus, the reconstruction algorithm iterates between real space and reciprocal space to find a set of variables that satisfy the real space constraint and the modulus constraint in the frequency domain. The schematic of a typical reconstruction loop is shown in Fig. 1.2.
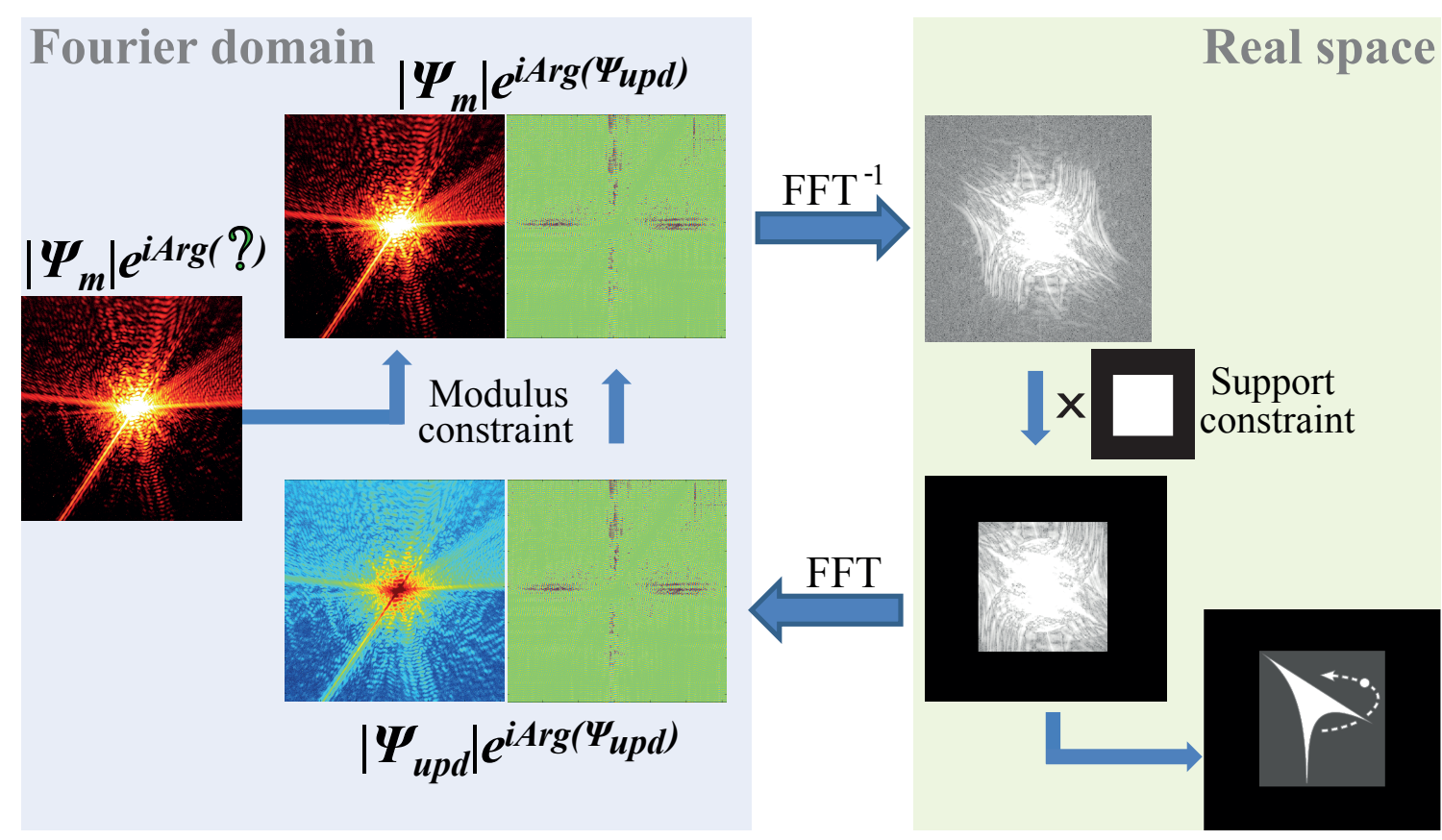

Figure 1.2: Schematic representation of a phase retrieval algorithm in CDI. The algorithm iterates between Fourier domain and real space via a fast Fourier transformation (FFT). In real space, a set of constraints is applied to find a projection $\Psi_{\text {upd }}$ onto the support constraint. In Fourier domain, the updated magnitudes, $\left|\Psi_{u p d}\right|$, are replaced with the measured magnitudes $\left|\Psi_{m}\right|$, while keeping the updated phase.

Due to experimental noise and the discretization of the signal during data recording and 
data processing, it is improbable to find a set of variables that will fully satisfy the constraints in both domains. To estimate the convergence and to terminate the reconstruction loop, the algorithm uses the ratio between the projection of the current solution onto the constraint and the constraint itself. Thus, the algorithm computes and monitors an error in real space or in the Fourier domain by comparing the corresponding step before and after the constraint is applied. For example, the real space error can be represented by the ratio between integrated values of the known-valued variables to the expected values. If the error value is not improving after a reasonably large number of iterations (typically between 1500 and 2000), the reconstruction algorithm has found the global error minimum corresponding to the solution, or it stagnated at a local minimum and the reconstruction loop can be stopped. The ability of a phase retrieval algorithm to avoid stagnation and the temporal evolution of the error are the most important characteristics of the algorithm.

There are several reconstruction algorithms that proved to be useful for a reconstruction of experimental data. Among them are the Error Reduction (ER) [3], Hybrid Input-Output (HIO) [4], difference map [50], solvent flipping [51], Relaxed Averaged Alternating Reflections (RAAR) [52] and oversampling smoothness [53] algorithms. The main difference between these algorithms is the way the real-space projection is computed, i.e, the way the solution guess is forced to be consistent with the real space support. For example, in the ER algorithm, values of the known-valued variables (pixels outside the support) are set exactly to expected values. In the case of an opaque mask, the values are set to zero. Thus, the algorithm is focusing to the nearest local minimum. In contrast, in the HIO algorithm, the known-valued pixels are not set to the expected values directly at the current iteration, but rather forced to reach the expected values in the following step. With this feedback loop, HIO is immune to stagnation at a local minimum and the reconstruction algorithm finds another minimum easily. The final output in this case can be an averaged solution over several minima or a minimum with the smallest error - presumably the global minimum.

When the illuminating beam is confined with a very small aperture right in front of the sample, CDI can be applied to extended objects [54, 55, 56, 57]. However, the conventional scheme allows for investigation of isolated objects only. The size of the object is essentially limited by the spatial coherence of the probe beam. Clearly, photons scattered from one edge of the object will interfere consistently with light from the opposite edge, if the lateral size of the object is smaller than the coherence length. Unlike in lens-based microscopy, lensless imaging cannot vary the magnification, or take an overview picture 
and afterwards zoom in the specific region. Instead, the real space image of the object is reconstructed with a real-space pixel size $d$ and a spatial resolution corresponding to the experimental parameters (numerical aperture and illumination wavelength) and the accuracy of the phase retrieval. The fundamental limit of the resolution is determined by the illuminating wavelength and the numerical aperture of the detection system (cf. Eq. 1.1). Additionally, the real-space pixel size of the reconstructed image is geometrically defined in each direction as $d=\frac{z \lambda}{p N}$, where $N$ is the number of pixels in the corresponding dimension, $\lambda$ is the illuminating wavelength, $z$ the distance between the sample and the detector and $p$ is the pixel size of the detector. For given experimental conditions (CCD parameters and illuminating wavelength), the real space pixel size $p$ can only be reduced when the diffraction data is recorded at a shorter distance $z$. On the other hand, by reducing this distance, the geometrical oversampling, $O$, will also decrease, where:

$$
O=\frac{z \lambda}{p D}
$$

The oversampling is directly related to the ratio between the total number of pixels to the unknown-valued pixel number, and therefore, reflects the ability of a phase retrieval algorithm to reconstruct the real space image [58, 59], since additional redundancy is required to compensate for experimental uncertainties and limitations. Thus, the distance between the sample and detector is chosen as a tradeoff between the desired resolution and the required oversampling in order to achieve a successful noise-free reconstruction. In principle, the resolution in CDI is limited only by the highest spatial frequency that is detected with a reasonable signal-to-noise ratio and sufficient oversampling. Additional information can be gained by recording a sequence of diffraction patterns obtained after illumination of the sample at different points with a spatially confined probe. The overlap between the probe areas provides redundancy for the phase retrieval process significantly improving its convergence [54]. This technique, commonly known as ptychographic CDI or ptychography, became a very popular extension to the conventional CDI scheme due to its robustness and relaxed requirements on a priori knowledge [60, 61, 62, 63, 64]. Furthermore, ptychography allows for reconstruction of the complex-valued probe at the sample plane.

Since the first experimental demonstration using synchrotron radiation by Miao et al. [67], lensless imaging has become a widely used technique that employs the full potential of modern sources of coherent radiation, such as free electron lasers and com- 

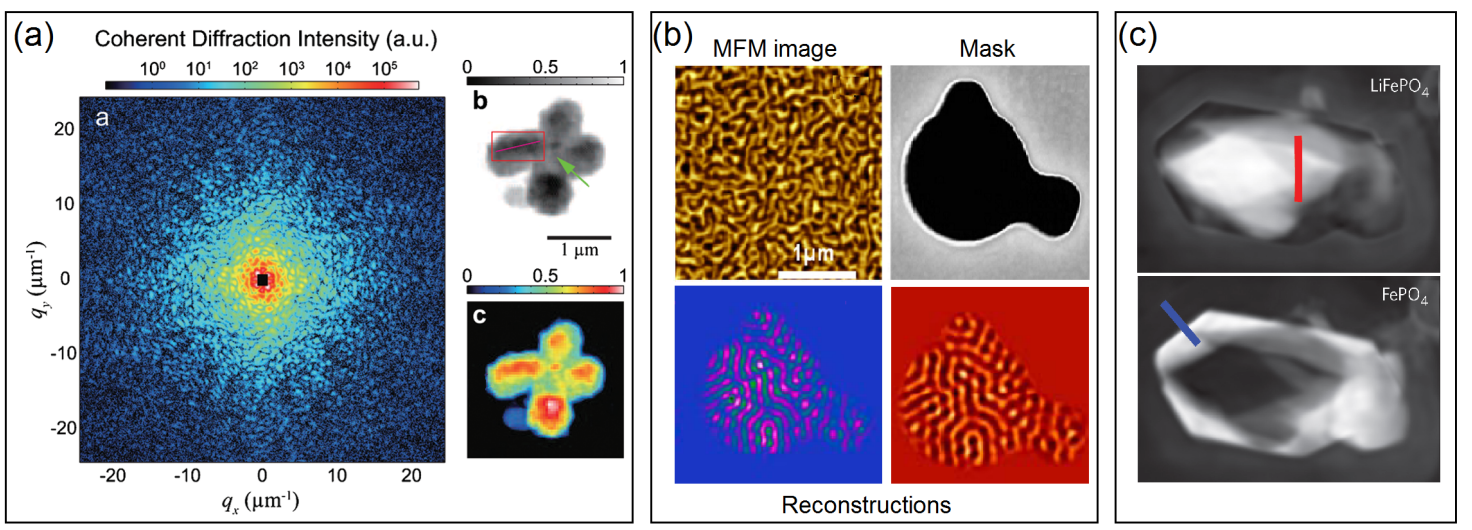

Figure 1.3: State of the art lensless imaging capabilities. (a) CDI of an unstrained human chromosome. Reprinted with permission from Ref. [65]. Copyright (2009) by the American Physical Society. (b) Lensless imaging of magnetic domains using resonant magnetic scattering. Reprinted with permission from Ref. [66]. Copyright (2012) by the American Physical Society. (c) Chemical composition mapping with soft X-ray ptychographic CDI. Reprinted by permission from Macmillan Publishers Ltd: Nature Photonics (Ref. [62]), copyright (2014).

pact EUV sources based on a principle of high-harmonic generation (HHG) [12]. Taking the advantage of immediate access to the comprehensive information of the exit wave, coherent diffractive imaging enables nanoscale mapping of diverse samples exhibiting any type of phase and amplitude contrast including chemical and elemental composition $[62,68,69,70]$, magnetic contrast $[71,66,72,73]$ and strains in complex matter $[74,75,76]$ with resolution down to a few nanometers [77, 62]. Because optical elements are not necessary, lensless imaging techniques are directly applicable for the entire electromagnetic spectrum including imaging at, e.g, a variety of resonant and absorption edges or at the water window [61], which are highly relevant for material science and biological applications ${ }^{3}$. Benefiting from the high penetration depth of X-rays together with an access to 3D information on the specimen via a phase retrieval process [78], CDI has been successfully implemented for noninvasive high resolution imaging of biological samples that are too thick for electron tomography, e.g., imaging of the whole yeast spore [79], unstained human chromosome [65] or virus cells [69, 10]. In terms of spatial resolution, coherent diffractive imaging, even with hard X-rays, is inferior to electron microscopy. However, several advantages in other aspects make CDI with X-rays not

\footnotetext{
${ }^{3}$ Water window is a part of electromagnetic spectrum between absorption edges of carbon at a wavelength of $4.4 \mathrm{~nm}$ and oxygen at $2.3 \mathrm{~nm}$ where the relative transparency of water allows for investigation of biological species including living cells and proteins in their natural aqueous environment.
} 
only an alternative microscopy method, but an unavoidable approach: the exposure time $[80,6,81]$ and temporal resolution can be in the range of tens of femtoseconds [13]; the sample thickness is not limited by the low penetration depth of electrons, allowing for imaging of relatively thick and buried samples; the lack of charging effects extends the applicability of CDI to non-conductive samples, and due to low absorption of X-rays by the atmosphere, also enables study of living, volatile and non-vacuum proofed specimens. It has also been shown that CDI can be successfully extended to the reflection geometry $[82,83,63,84]$. Furthermore, no labeling techniques are required, as in the case of superresolution optical microscopy [85], to resolve features of tens of nanometers. In fact, the imaging resolution of biological samples is limited by the X-ray dose, whereas an ultrashort and intense X-ray probe, e.g., a pulse from free electron lasers can, in principle, improve imaging resolution to less than $1 \mathrm{~nm}[86]$.
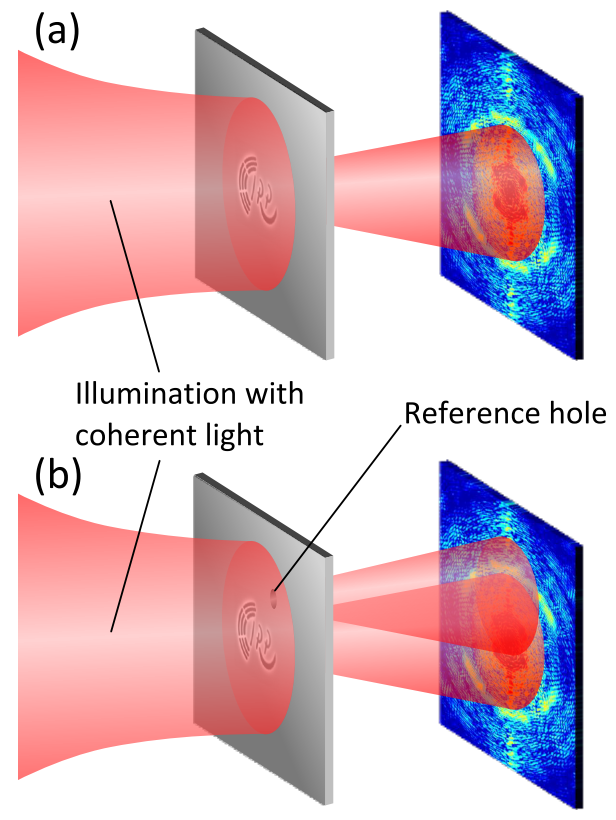
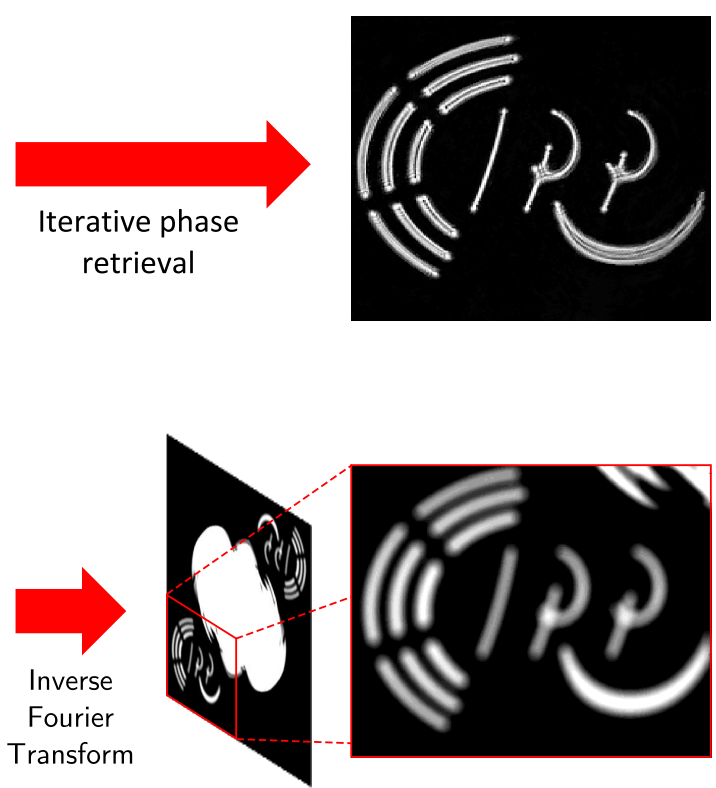

Figure 1.4: Schematic of lensless imaging experiments. (a) Coherent diffractive imaging. The lost phase information is recovered via an iterative phase retrieval algorithm. (b) Fourier transform holography. The phase of the scattered signal is already encoded into the interference pattern between the sample and a reference beam.

The information on the phase of the scattered beam at a detector plane can also be obtained experimentally using the interference of the scattered beam with some known field, 
for example, a flat-field from a reference source. The imaging technique known as Fourier transform holography (FTH) is based on this principle. A typical experimental scheme for FTH is illustrated in Fig. 1.4(b). Here, the reference beam is produced by the illumination of a very small aperture (a reference hole) in the sample plane at the distance greater than the size of the object. Using the inverse Fourier transform of the measured far-field intensity distribution, cross-correlations between the scattered signal from the object and the reference hole are accessible in a single step. Using this technique, the resolution of the cross-correlation reconstructions is determined by the size of the reference beam in the sample plane, i.e., the size of the reference hole itself (assuming that the reference signal covers the entire detector area). However, a low signal level from a very small reference hole will reduce the contrast of the reconstructed images, thus, the size of the reference holes is a tradeoff between the spatial resolution and the image contrast. Multiple reference holes might help to increase the image contrast by averaging the final reconstruction over individual cross-correlations ${ }^{4}$ [87]. Some improvement was also demonstrated using a Fresnel zone plate to create a well-defined reference beam with much higher flux density [88] and an approach utilizing the extended references [89, 90, 43].

Major requirements for FTH are similar to CDI, including high spatial and temporal coherence of the probe, spatial isolation of the scattered signal and sufficient sampling, determining the real pixel size of the reconstructions. Additionally, it is necessary that the signal from the reference holes(s) covers the entire detector, otherwise high spatial frequencies scattered from the investigated object will not fully contribute to the FTH reconstruction and, therefore, reduce the achievable resolution. Despite weaker performance of FTH compared to CDI in terms of the spatial resolution, the simplicity of the FTH reconstruction process and its robustness, in some cases, can be a substantial advantage. The resolution of holographic reconstructions can be refined by applying an iterative phase retrieval algorithm to the measured far-field holography [91, 92, 93]. However, in this case, the oversampling and probe's coherence requirements must be fulfilled for the entire field of view, including the investigated object and all reference holes.

\footnotetext{
${ }^{4}$ Each reference hole produces a cross-correlation image and its complex conjugate.
} 


\subsection{Probes for lensless imaging}

Coherent short wavelength radiation from new generation synchrotrons, free electron lasers (FEL) and compact high-harmonic generation (HHG) sources fulfill the requirements for high resolution imaging with lensless techniques introduced in Chapter 1.2. The first two aforementioned types of EUV/X-ray sources rely on the acceleration (deflection) of charged particles by strong magnetic fields, whereas HHG sources are based on a principle of frequency up-conversion. Large scale synchrotron facilities give access to tunable radiation in the wide spectral range from microwaves to hard X-rays, with coherence properties typically provided by grating-based monochromators and by spatial confinement with a pinhole or a set of slits. Synchrotron radiation is delivered in the form of short pulses with repetition rates up to a few $\mathrm{MHz}$, mitigating high losses associated with the spatial confinement and ensuring relatively high photon flux at the sample. In contrast to synchrotron facilities, FELs support smaller energy ranges and operate at much lower repetition rates, typically a few Hz. However, a tremendous increase of the photon flux in FELs results in an increased peak brilliance by up to 10 orders of magnitude compared to synchrotrons with the highest brilliance [94]. Furthermore, radiation emerging from an FEL is spatially and temporally coherent and, in principle, can be directly used for lensless imaging experiments. The improved pulse duration reaches values below 100 femtoseconds, and, together with high brilliance, enables single shot CDI measurements with femtosecond exposure times [6], where the temporal resolution is mainly limited by the timing jitter to a few picoseconds [80]. With newly proposed seeded-FEL schemes for synchronization of the arrival time, the timing jitter can be improved to reach the femtosecond range [95]. Despite the great potential of these new generation synchrotrons and FELs, only a few facilities are available worldwide, partially because of their enormous size and cost. In contrast, radiation from a compact high-harmonic generation source can possess remarkable properties already on a tabletop, including a broad spectrum up to high photon energies, full spatial coherence, the shortest available pulse duration and the best possible temporal resolution.

\subsection{High-harmonic generation}

Nonlinear frequency conversion of a fundamental frequency into its second harmonic was first observed in quartz in 1961 shortly after development of the operating laser [96]. 
Later, the generation of higher order harmonics was extended to the 5th order in gases [97]. Using perturbation theory, frequency conversion in those experiments can be described as a multi-photon excitation, where the photon absorption probability exponentially decreases with the photon number. Thus, the conversion efficiency becomes very low and generation of higher harmonics is not feasible.

(a)

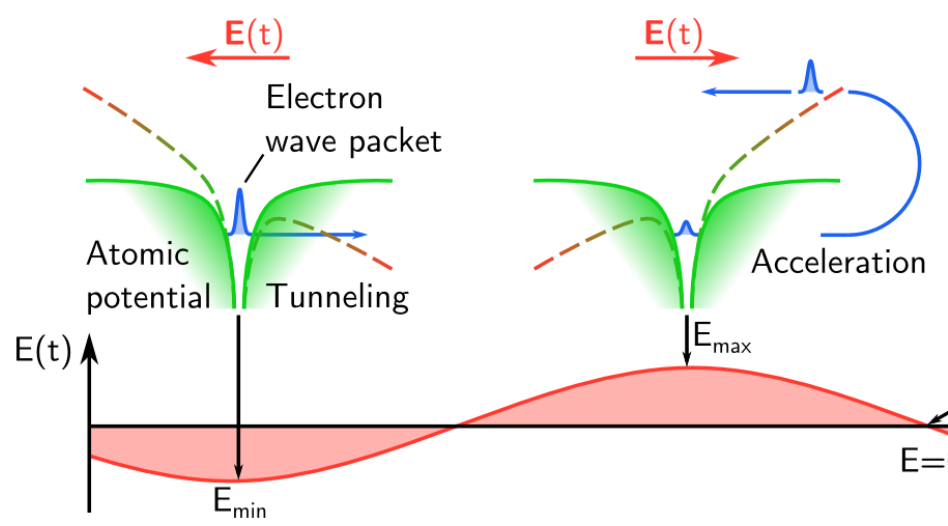

(c)

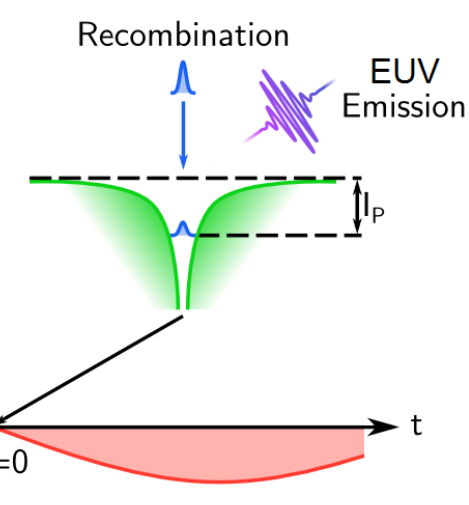

(d)

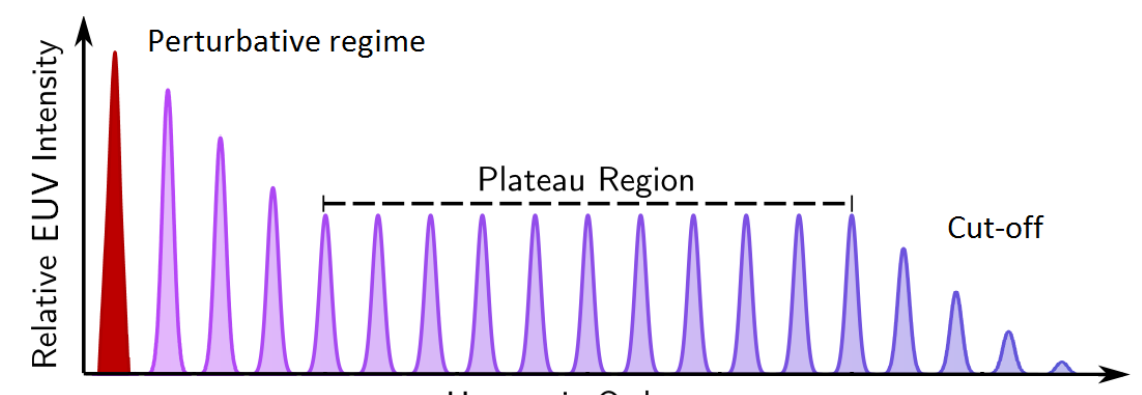

Harmonic Order

Figure 1.5: Schematic representation of the three step model describing the high-harmonic generation process: (a) Tunnel ionization; (b) Acceleration in the laser field; (c) Recombination and photoemission. (d) Typical high-harmonic generation spectrum on a logarithmic scale.

The development of pulsed lasers with very high peak power and peak intensities exceeding $10^{13} \mathrm{~W} / \mathrm{cm}^{2}$ allowed for the generation of higher-order harmonics [12, 98]. A typical high-harmonic spectrum in a noble gas is illustrated in Fig. 1.5(d). It includes three distinct features: a rapid intensity decrease at order harmonics, followed by a flat plateau with harmonics of comparable intensity, that sharply ends at a cut-off energy. While the fast signal decrease of the low order harmonics is consistent with the nonlinear optical perturbation theory, the plateau region could not be explained within this framework. 
To estimate the maximum photon energy that can be obtained via the HHG process, i.e., the harmonic of the cut-off energy, J. Krause and co-workers calculated the optical harmonic spectra from the single-atom response. They developed a simple formula that for fields below the saturation intensity depends only on the frequency of the driving field $\omega_{0}$ and the ionization potential $I_{p}$ of the gas [99]. The maximum photon energy at the cut-off is given by:

$$
E_{\text {cutoff }} \approx I_{p}+3 U_{p}
$$

where $U_{p}=I / 4 \omega_{0}^{2}$ is the ponderomotive potential (in atomic units), which corresponds to the quiver energy of the electron gained in an electromagnetic field of intensity $I$ and oscillating frequency $\omega_{0}$. Thus, for a given gas species, the cutoff energy is proportional to the square of the laser wavelength $\lambda$ and the laser intensity, i.e., $E_{\text {cutoff }} \propto I \lambda^{2}$. This scaling is consistent up to the saturation intensity, where the medium is strongly ionized and plasma effects, such as dephasing and defocusing, can not be properly controlled. Employing such a wavelength dependent cutoff law, T. Popmintchev and co-workers demonstrated that high-harmonic radiation can be extended to sub-nm wavelengths using a laser light with lower frequency to drive the HHG process [23]. Figure 1.6 (reprinted from Ref. [23]) shows the experimental spectra of high harmonics generated in He using a different driver wavelengths from $0.8 \mu \mathrm{m}$ to $3.9 \mu \mathrm{m}$ available via optical parametric amplification laser systems. Here, the generated emission from $3.9 \mu \mathrm{m}$ driver emerge as a bright supercontinuum, covering 12 octaves of bandwidth via extreme orders of highly nonlinear process.

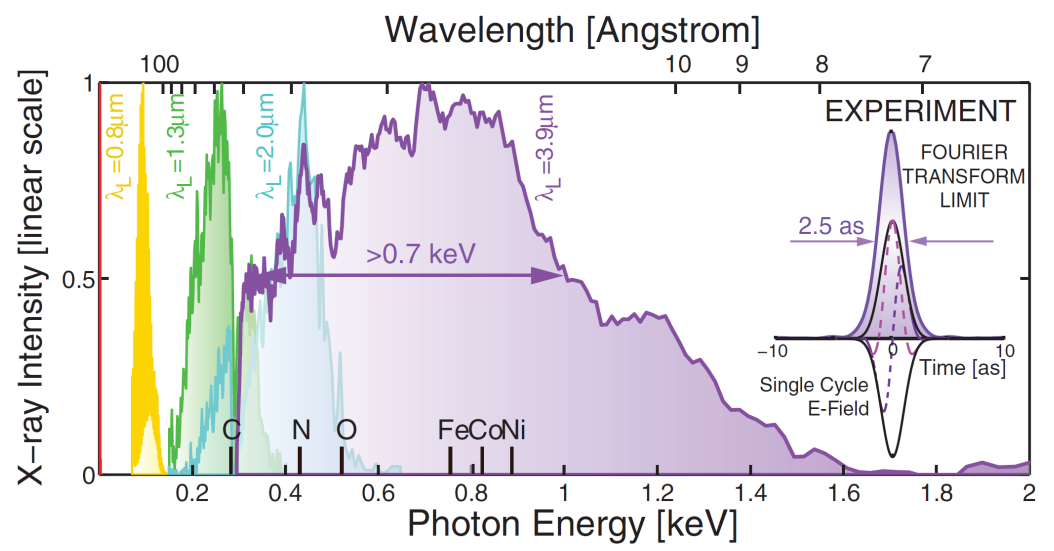

Figure 1.6: Experimental spectra of high-harmonics as a function of driving-laser wavelength representing the microscopic cutoff rule. From Ref. [23]. Reprinted with permission from AAAS. 


\subsubsection{Three-step model}

In 1993, Paul Corkum proposed a simple theoretical description of the HHG process induced by intense laser fields [100], which explains the most important features of a high-harmonic spectrum: the plateau and the cut-off. For that, the quasi-static model of multiphoton ionization [101] was extended to account for the electron interaction with its parent ion after ionization in gaseous media. The proposed model represents the electronion interaction semi-classically using three distinct steps, as illustrated in Fig. 1.5(a-c):

(1) Tunneling of an electron from its binding Coulomb potential;

(2) Classical free space motion of the electron in the laser field;

(3) The recollision with the parent ion with recombination to the ground state, accompanied by emission of a high frequency photon.

Step 1. Tunnel ionization Figure 1.7 illustrates three possible cases of the laser field interaction with an atom. At relatively low field intensities, the binding energy of the electron is much lower than the photon energy of the laser, and ionization of the atom can be described through the multiphoton ionization process [cf. Fig.1.7(a)]. For moderate laser intensities typically exceeding $10^{14} \mathrm{~W} / \mathrm{cm}^{2}$ the binding atomic potential is strongly distorted (bent) by the laser field. Thus, a valence electron has a nonzero probability to tunnel through the potential barrier of the strongly distorted atomic potential.The instantaneous tunneling probability depends on the "shape" of the barrier, which is governed by the electric field strength, whereas the overall tunneling probability requires integration over time (within a half-optical cycle) for which a sufficient barrier suppression is achieved. This limits the laser frequency, $\omega_{0}$, that can fulfill the quasi-static approximation.

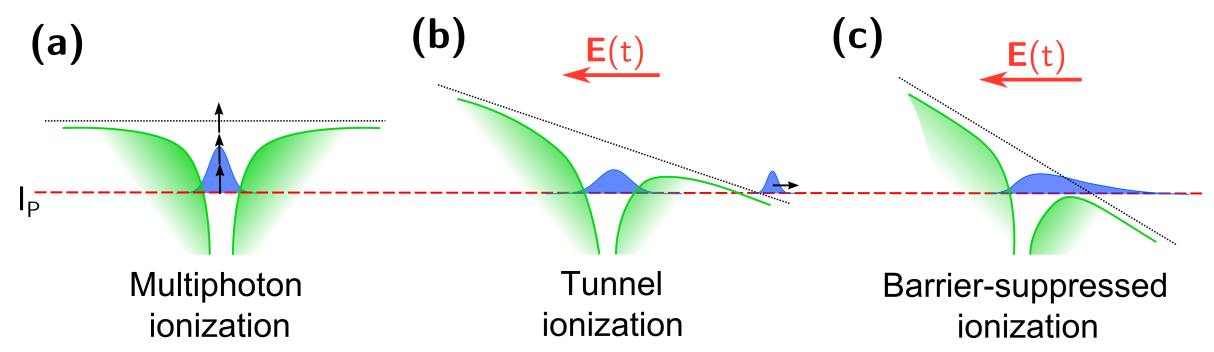

Figure 1.7: Possible ionization mechanisms.

When the laser intensity is further increased, the binding potential is drastically suppressed and electrons from the ground state can classically escape from the atom. This 
regime is also known as barrier-suppressed ionization (cf. Fig. 1.7). Interestingly, a stronger barrier suppression leads to an increased probability of the tunneling ionization of already ionized atoms, i.e., the liberation of inner shall electrons with a much higher ionization potential.

To define the ionization type, it is convenient to use the so-called Keldysh parameter $\gamma$, that distinguishes between the tunneling ionization $(\gamma \ll 1)$ and the multiphoton regime $(\gamma \gg 1):$

$$
\gamma=\sqrt{\frac{I_{p}}{2 U_{p}}} .
$$

Step 2. Acceleration in the laser field The second step of the semi-classical model represents a propagation of the liberated electron. This step is well described classically as acceleration of the free electron in the presence of a strong electric field from zero initial velocity. The Lorentz force can be neglected, since the electron does not reach relativistic speeds within an optical half cycle. The influence of the Coulomb potential after tunneling is also negligible compared to the laser field. When the electric field changes its sign after half of the optical cycle, the electron is accelerated back towards its parent ion. The energy gained by the electron in the electric field is released as a highenergy photon, when the electron recombines with the ion. This energy depends on the electron's trajectory, specifically, on the final kinetic energy gained by the electron in free space before recollision, and therefore on the tunneling phase relative to the phase of the electric field of the laser. Most of the tunneled electrons will not recombine due to an unfavorable tunneling phase. The most energetic trajectory - the trajectory that allows electrons to reach the highest kinetic energy before recollision - produces photons with $E_{\text {cutoff }}$ energy. Trajectories with non-zero recollision probability emerged before and after the most energetic trajectory (within the same optical half cycle) are called "short" and long "trajectories", accordingly. Varying the experimental conditions, the HHG process can be tuned to favor short or long trajectories which allows to control the coherence and divergence properties of the high-harmonic emission [24].

Step 3. Recollision and photoemission The final step of the three-step model is the recollision of the electron with the parent ion. Here, as mentioned earlier, the kinetic energy gained by the electron in the electric field is added to the ionization potential and 
released in a harmonic photon, whose energy depends on the tunneling phase. A proper interpretation of the recollision step requires a quantum mechanical treatment, and can be described as a transition to the autoionizing sate with subsequent relaxation to the initial (ground) state accompanied by the EUV emission [102]. The electron recombines with the initial state, because the returning electron wavepacket is overlapped with the nuclear wavepacket.

This three step process repeats every half cycle at the leading edge of a laser pulse, resulting in deterministic photon emission from many gas atoms within a very short time, provided that the phase of the driver is favorable for tunneling ionization. Since the harmonics are emitted within a fraction of the fundamental pulse, the EUV pulse duration is much shorter than the pulse length of the driver and, therefore can reach attoseconds range [103]. As the laser pulse propagates through the gas medium, high-harmonics are emitted with a very similar phase and therefore inherit coherence properties of the fundamental laser light.

\subsubsection{Phase matching}

To ensure high conversion efficiency of the laser light to high harmonics, the emission from every responding atom at the leading edge of the fundamental pulse must contribute constructively to build up with the propagation of the laser along the interaction region. This can be achieved, if dispersion effects are minimized and the phase velocity of the generated harmonics is equivalent to the phase velocity of the fundamental radiation, i.e., the refractive index $n$ of the EUV radiation must be equal to that experienced by the fundamental frequency. In this case, the phase mismatch of the wave vector $k$ is zero $(\Delta k=0)$. Finding experimental conditions, at which the phase matching conditions are fulfilled for substantial propagation distance, is of utmost importance in experiments involving harmonic generation.

Conventional phase matching techniques, for example, frequency doubling in crystals, are based on anisotropic materials, where the refractive index depends on the polarization. In this case, $\Delta k$ can be minimized if the fundamental frequency and generated emission have different polarizations. In contrast, a gas medium is isotropic and conventional phase-matching schemes are not applicable.

For the free space propagation of intense laser pulses in the gas medium, the projection 
of the $k$-vector onto the propagation direction is described by:

$$
k \approx \frac{2 \pi}{\lambda}+\frac{2 \pi N_{a} \delta(\lambda)}{\lambda}-N_{e} r_{e} \lambda
$$

where $N_{a}$ and $N_{e}$ are the atom and electron densities, respectively, $\delta(\lambda)$ represents the dispersive characteristics of neutral atoms ${ }^{5}, r_{e}$ is the classical electron radius and $\lambda$ is the driving wavelength $[104,105]$. The first term in equation 1.6 corresponds to the wave vector in vacuum, whereas the second and the third terms represent contributions from the gas medium (neutral atoms) and free electrons dispersion due to plasma formation. Fortunately, the dispersion of gas and plasma have an opposite sign, allowing for tuning the $k$-vector of the driver by controlling the ratio between neutral atoms and free electrons from the plasma. Since the phase velocity of high-harmonics is not affected as strongly as the phase velocity of the driver, experimental conditions for $\Delta k=0$ can be found. Note that Eq.1.6 does not account for the Gouy phase shift that a convergent beam experiences in the focus region, for example, when high harmonics are generated before or after the focus [106]. The phase mismatch for the harmonic order $q$ can be written as [107]:

$$
\Delta k \approx P \eta q N_{a} r_{e} \lambda-(1-\eta) \frac{2 \pi P q}{\lambda} \Delta \delta .
$$

Here, $P$ is the gas pressure, $\eta$ is an ionization level and $\Delta \delta$ is the difference between the refractive indices of the driver and the harmonic wavelength in the gas medium. The phase matching condition $(\Delta k=0)$ can be achieved by tuning the gas pressure at the interaction region, balancing between the plasma dispersion (first term in Eq. 1.7) and neutrals (second term in Eq. 1.7). However, effective balancing of the plasma dispersion with neutral atoms is possible only until the ionization level $\eta$ reaches its critical value, limiting efficient HHG to ionization levels $<5 \%$.

Apart from the coherence length - a propagation distance at which $k$ reaches value of $\pi$, another limiting factor for HHG efficiency in a gas jet can be the decreasing laser intensity caused by the divergence angle of the laser light after the focus. To overcome this limitation, the interaction can be performed in a hollow core waveguide [108]. In this case, an additional term in Eq. 1.7 corresponding to the geometrical dispersion needs to be considered. The beam profile and conversion efficiency of high harmonics generated in the fiber have a potential to be further improved due to a more constant curvature

\footnotetext{
${ }^{5}$ Linear wavelength dependent refractive index per unit neutral atom density, minus 1.
} 
of the driver's wavefront along the waveguide capillary and longer interaction lengths $[105,108]$. It has been reported that the hollow-core generation geometry enables full spatial coherence [109] and improves the conversion efficiency by several orders of magnitude [105] compared to a conventional self-guiding scheme. However, later it has been demonstrated that a full spatial coherence can be also achieved without using a waveguide [110], and that conventional generation schemes utilizing a gas-filled capillary (gas cell) or even a gas jet provide comparable conversion efficiency as well as a high EUV photon flux [111, 32].

\subsubsection{Lensless imaging with high-harmonic radiation}

As mentioned earlier, high harmonic radiation exhibits essentially full spatial coherence, which is ideally suited for lensless imaging experiments. The first experimental implementation of high harmonic radiation for lensless imaging ${ }^{6}$ was demonstrated in 2007 by R. Sandberg and co-workers [112]. In their experiment, phase matching conditions of the HHG process were optimized in a hollow core waveguide, ensuring low divergent radiation with high spatial coherence. Figure 1.8, adapted from the original publication of Sandberg et al. [112], illustrates the experimental setup used (top row) and first results of CDI with HHG source(bottom row). To isolate a single harmonic and to increase the temporal coherence required for CDI, a pair of narrow-band multilayer mirrors centered at $29 \mathrm{~nm}$ was used. A spectral bandwidth at the sample was estimated as $\lambda / \Delta \lambda>200$. Several diffraction patterns with a variable beam-block size were patched together to increase the dynamic range of the diffraction data used for reconstruction. A spatial resolution of $214 \mathrm{~nm}$ was demonstrated using a knife-edge measurement for a reconstruction with a real pixel size of $107 \mathrm{~nm}$, limited by the low numerical aperture used. Although the generated flux was estimated to be $\sim 10^{12}$ photons/second, the measured flux at the sample position was below $10^{8}$ photons/second. To compensate such losses, exposure times of more than two hours were required [113].

Subsequent improvements of the source stability and utilization of high numerical apertures enabled imaging with resolution twice better than previously demonstrated - down to $1.5 \lambda$ [114]. It has been shown, that for high numerical apertures, the diffraction data should account for distortions arising from the detection of the spherical wave with a

\footnotetext{
${ }^{6}$ The applicability of high-harmonic radiation for lensless imaging was first demonstrated using Gabor holography technique with spatial resolution of $\sim 7 \mu \mathrm{m}$ [109].
} 


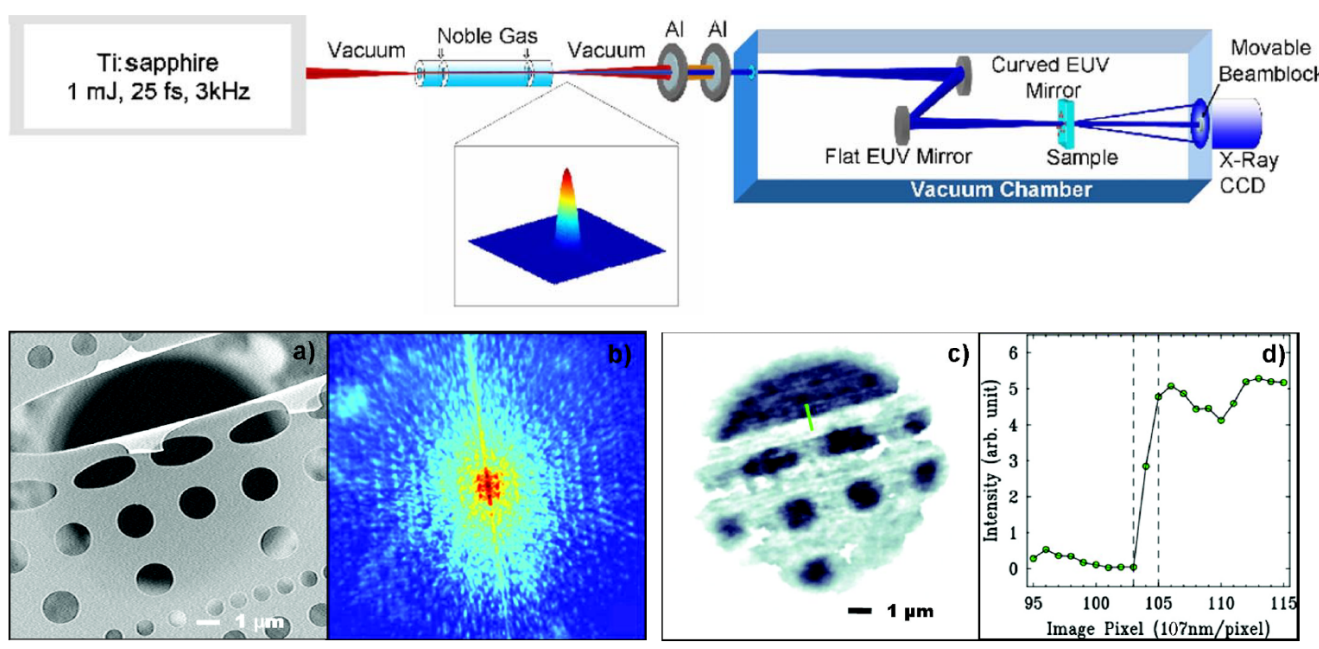

Figure 1.8: First experimental demonstration of coherent diffractive imaging using high harmonics. Reprinted with permission from Ref. [112]. Copyright (2007) by the American Physical Society.

flat detector, i.e., to interpolate the experimental data from a plane onto a spherical surface. Later HHG sources were successfully implemented for Fourier transform holography $(\mathrm{FTH})$ with sub $100 \mathrm{~nm}$ spatial resolution and reduced exposure times [92]. Using a very powerful laser facility, a single shot CDI as well as FTH were demonstrated with a spatial resolution of $110-120 \mathrm{~nm}$, and exposure times as short as $20 \mathrm{fs}[13,90]$. Furthermore, new approaches for multiple wavelength diffractive imaging allow to utilize the entire spectrum of the emitted harmonics [115], as well as a broadband probe [116], which might improve the exposure times at the costs of spatial resolution. Using a state-of-theart high harmonic generation source, coherent diffractive imaging at $13 \mathrm{~nm}$ wavelength was demonstrated with a spatial resolution of $25 \mathrm{~nm}$ (measured using a knife-edge technique) with just 30 seconds of exposure time [117]. 


\title{
Chapter 2
}

\section{Toroidal grating astigmatism of high-harmonics characterized by EUV Hartmann sensor}

Tobias Mey, Sergey Zayko, Claus Ropers, Bernd Schäfer, and Klaus Mann Optics Express, Vol. 23, 15310-15315 (2015)

DOI: 10.1364/OE.23.015310

\begin{abstract}
The beam transport of single high-order harmonics in a monochromator arrangement is studied. A toroidal grating combines spectral filtering and focusing in order to produce a small individual spot for each harmonic. Here, the effect of small deviations from perfect alignment is investigated. Experimentally, a Hartmann sensor monitors the EUV wavefront while the grating is subjected to an online alignment procedure. The obtained results are confirmed by a simple theoretical description employing optical matrix methods.
\end{abstract}

The high-harmonic generation (HHG) process converts electromagnetic radiation into integer multiples of the fundamental frequency reaching EUV and soft $\mathrm{x}$-ray wavelengths $[12,74]$. All individually quasi-monochromatic harmonics propagate collinearly with the incident laser beam. In order to prepare a beam which contains only a few or even single harmonics, multilayer mirrors with a wavelength-selective reflectivity are commonly used [118]. Similarly, a reflection grating acts on the HHG beam by separating the harmonics into different diffraction angles [119]. Both techniques can be combined with curved surfaces to refocus the divergent $\mathrm{HHG}$ radiation to a probe position. However, such devices are highly sensitive to slight misalignments of, e.g., the angle of incidence. As a result, aberrations lead to an increased focal spot diameter and a reduced intensity. Moreover, the 
phase distribution becomes distorted, which limits the applicability of the EUV radiation in phase retrieval applications such as coherent diffractive imaging. This creates the need for a highly precise alignment of the involved optical elements.

Hartmann type sensors are known to be versatile tools for optics alignment in the visible and UV spectral range [120,121]. The wavefront distribution is derived from single exposures in terms of a composition of specific aberrations. This allows for an online wavefront monitoring and thus for an adjustment of the related optics in the beam path. Recently, similar applications have been successfully demonstrated in the EUV range, too, where an ellipsoidal focusing optic is aligned with an unprecedented precision [122, 123].

In this work, the propagation of single harmonics is studied after passing a toroidal grating that combines spectral filtering and focusing. A Hartmann sensor captures the EUV wavefront while the angle of incidence of the harmonic on the grating is varied. Here, already tiny variations lead to a significant impact, especially influencing the astigmatic aberration. Beyond the experimental characterization, the observed effect is described theoretically by matrix methods. Both methods agree well to each other and an optimum angle of incidence is found, at which aberrations are minimized.

\section{Experimental}

The setup of the investigated HHG source is schematically depicted in figure 2.1. A titanium sapphire laser generates ultra-short pulses of near-infrared radiation which are focused into an argon filled capillary (pulse energy $0.5 \mathrm{~mJ}$, pulse length $40 \mathrm{fs}$, center wavelength $800 \mathrm{~nm}$, repetition rate $1 \mathrm{kHz}$ ). In the noble gas, the highly non-linear HHG process results in odd harmonics, measured up to the 51st order. After the conversion, the fundamental beam is absorbed by a mesh-less aluminum filter of $200 \mathrm{~nm}$ thickness, whereas radiation in the wavelength range $17 \mathrm{~nm} \lesssim \lambda \lesssim 80 \mathrm{~nm}$ is transmitted [124]. A toroidal grating generates a row of individual foci for each harmonic (sagittal radius $R_{s}=104.9 \mathrm{~mm}$, tangential radius $R_{t}=1000 \mathrm{~mm}$, groove density $g=550 \mathrm{~mm}^{-1}$, focal length in 0 . order $f_{\text {tor }}=160 \mathrm{~mm}$, manufactured by Jobin Yvon Inc.). In the -1 . diffraction order, a slit allows for propagation of single harmonics while all others are blocked. Within the scope of the present study, the 25th harmonic is selected, corresponding to a wavelength of $\lambda=32 \mathrm{~nm}$. 


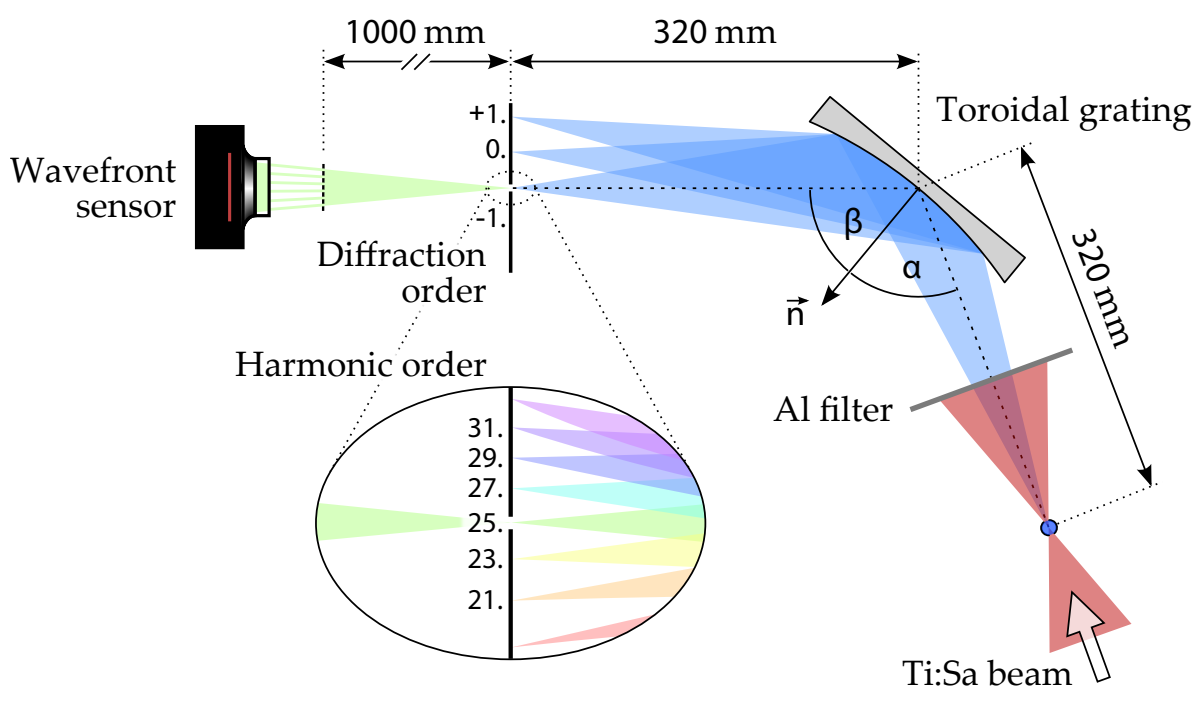

Figure 2.1: Setup of HHG source with a single toroidal grating acting as monochromator. $\vec{n}$ denotes the normal vector of the toroidal surface.

The Hartmann sensor is a combination of a CCD chip $(1392 \times 1040$ square pixels, size $6.5 \mu \mathrm{m}$, dynamic range $14 \mathrm{bit}$ ) with a pinhole array in a distance of $95 \mathrm{~mm}$ to the chip (pinhole diameter $75 \mu \mathrm{m}$, pinhole pitch $250 \mu \mathrm{m}$ ). The detector is placed $1000 \mathrm{~mm}$ behind the expected focal position of the HHG beam. In order to accumulate a sufficient signal, an exposure time of $40 \mathrm{~s}$ is selected.

The EUV beam is divided by the pinhole array into many small sub-beams, which then individually propagate in the direction of the local Poynting vector, each producing a spot on the CCD chip. The wavefront is then reconstructed by a comparison between the resulting spot distribution and a reference distribution corresponding to a plane wave illumination with Gaussian spots separated by $250 \mu \mathrm{m}$ in the horizontal and vertical direction.

In case of coherent radiation, the information obtained by the Hartmann sensor allows for the calculation of beam propagation parameters, e.g., the horizontal and vertical waist positions [122]. In the following, the distance between both positions $\Delta z$, i.e., the waist difference, will be used as a measure for the astigmatic aberration. A detailed description of the principle of the employed sensor is found in Refs. [123, 125].

During online monitoring of the wavefront, the angle of incidence $\alpha$ between the HHG 
beam and the normal of the toroid is increased in steps of $\Delta \alpha=0.05^{\circ}$. The deflection angle $\beta$ changes according to the grating equation [126]

$$
\sin \beta-\sin \alpha=m g \lambda,
$$

with the diffraction order $m$ and groove density $g$. In the course of this alignment procedure, the effective radius of the optic decreases in the tangential direction, and it increases in the sagittal direction [127]. As a consequence, the beam experiences a stronger horizontal focusing and a weaker vertical focusing. For a certain angle $\alpha_{0}$, both focal distances are equal to each other and the foci coincide. A selection of three wavefronts captured at different angles $\alpha$ is depicted in Figure 2.2.

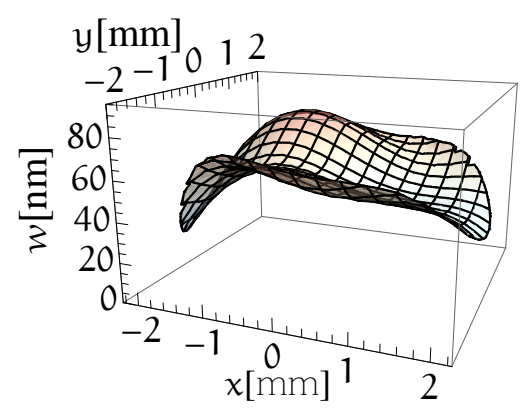

(a) $\begin{aligned} \alpha & =72.20^{\circ} \\ \Delta z & =27 \mathrm{~mm}\end{aligned}$

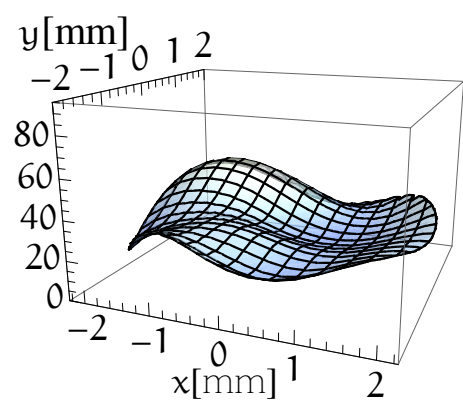

(b) $\begin{aligned} \alpha & =72.65^{\circ} \\ \Delta z & =0 \mathrm{~mm}\end{aligned}$

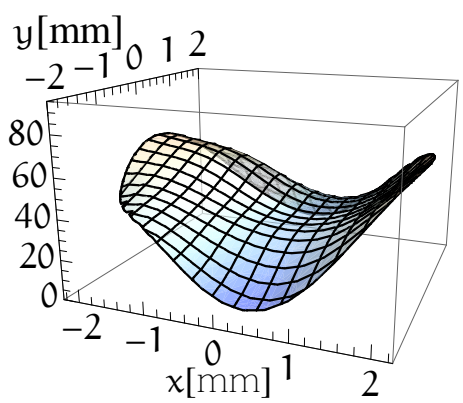

(c) $\begin{aligned} \alpha & =72.95^{\circ} \\ \Delta z & =-22 \mathrm{~mm}\end{aligned}$

Figure 2.2: HHG wavefront of the $25^{\text {th }}$ harmonic, measured at different angles of incidence $\alpha$. Tilt and defocus terms are subtracted, $\Delta z$ denotes the astigmatic waist difference.

Apparently, already small variations of the angle of incidence lead to a significant change of the astigmatic aberration. The curvature of the wavefront indicates that at an angle of incidence of $\alpha=72.20^{\circ}$, the beam is horizontally divergent and vertically convergent. At $\alpha=72.95^{\circ}$ the situation appears reversed. Thereby, the astigmatic waist difference $\Delta z$ changes its value from $27 \mathrm{~mm}$ to $-22 \mathrm{~mm}$. In between, at $\alpha=72.65^{\circ}$ the focusing of the optic is well balanced, and $\Delta z$ vanishes. However, the wavefront is not entirely flat due to remaining higher order aberrations.

\section{Theoretical description}

In the following, the astigmatic waist difference $\Delta z(\alpha)$ is theoretically estimated by matrix methods and then compared to the experimental results. 
The fundamental laser beam is taken to be Gaussian with a local diameter of $8 \mathrm{~mm}$ before being focused by the lens into the argon filled capillary (focal length $200 \mathrm{~mm}$ ). Thus, the divergence is $\approx 8 / 200=40 \mathrm{mrad}$ and with $M^{2}=1$, the focus diameter is derived to $d_{0}=25 \mu \mathrm{m}$ [128]. The 25th harmonic is assumed to exhibit the same source diameter and a divergence which is 25 times smaller than that of the incident laser beam:

$$
\begin{aligned}
d_{0} & =25 \mu \mathrm{m} \\
\theta & =1.6 \mathrm{mrad} .
\end{aligned}
$$

With these properties, the HHG beam matrix

$$
\mathscr{M}_{0}=\left(\begin{array}{cccc}
\left\langle x^{2}\right\rangle & \langle x y\rangle & \langle x u\rangle & \langle x v\rangle \\
\langle x y\rangle & \left\langle y^{2}\right\rangle & \langle y u\rangle & \langle y v\rangle \\
\langle x u\rangle & \langle y u\rangle & \left\langle u^{2}\right\rangle & \langle u v\rangle \\
\langle x v\rangle & \langle y v\rangle & \langle u v\rangle & \left\langle v^{2}\right\rangle
\end{array}\right)
$$

is derived at the source position, where all mixed moments vanish [129]. Now, ray transformation matrices as given in the appendix are applied to the beam matrix $\mathscr{M}_{0}$ corresponding to the experimental situation. First, $\mathscr{M}_{0}$ is propagated to the optic by $\mathscr{S}_{\text {prop }}(320 \mathrm{~mm})$. Then, the toroidal grating $\mathscr{S}_{\mathrm{tg}}(\alpha)$ acts on the beam and a subsequent propagation by $\mathscr{S}_{\text {prop }}(z)$ finally yields the system matrix $\mathscr{S}(z, \alpha)$ that transforms a beam $\mathscr{M}_{0}$ from the source position to a distance $z$ behind the optic:

$$
\begin{aligned}
\mathscr{S}(z, \alpha) & =\mathscr{S}_{\text {prop }}(z) \cdot \mathscr{S}_{\mathrm{tg}}(\alpha) \cdot \mathscr{S}_{\text {prop }}(320 \mathrm{~mm}) \\
\mathscr{M}(z, \alpha) & =\mathscr{S}(z, \alpha) \cdot \mathscr{M}_{0} \cdot \mathscr{S}(z, \alpha)^{T} .
\end{aligned}
$$

Here, the beam matrix is propagated to the detector position $z=1320 \mathrm{~mm}$. With $\mathscr{M}(1320 \mathrm{~mm}, \alpha)$ the waist positions $z_{0, x}(\alpha)=-\langle x u\rangle /\left\langle u^{2}\right\rangle$ and $z_{0, y}(\alpha)=-\langle y v\rangle /\left\langle v^{2}\right\rangle$ are derived, and the astigmatic waist difference $\Delta z(\alpha)=z_{0, x}(\alpha)-z_{0, y}(\alpha)$ is computed. The resulting function $\Delta z(\alpha)$ is depicted in figure 2.3 as a solid blue line together with the values resulting from the measured wavefronts.

The theoretical waist difference $\Delta z(\alpha)$ appears slightly above the experimental values. Although the relative angle of incidence is adjusted very precisely, its absolute value 


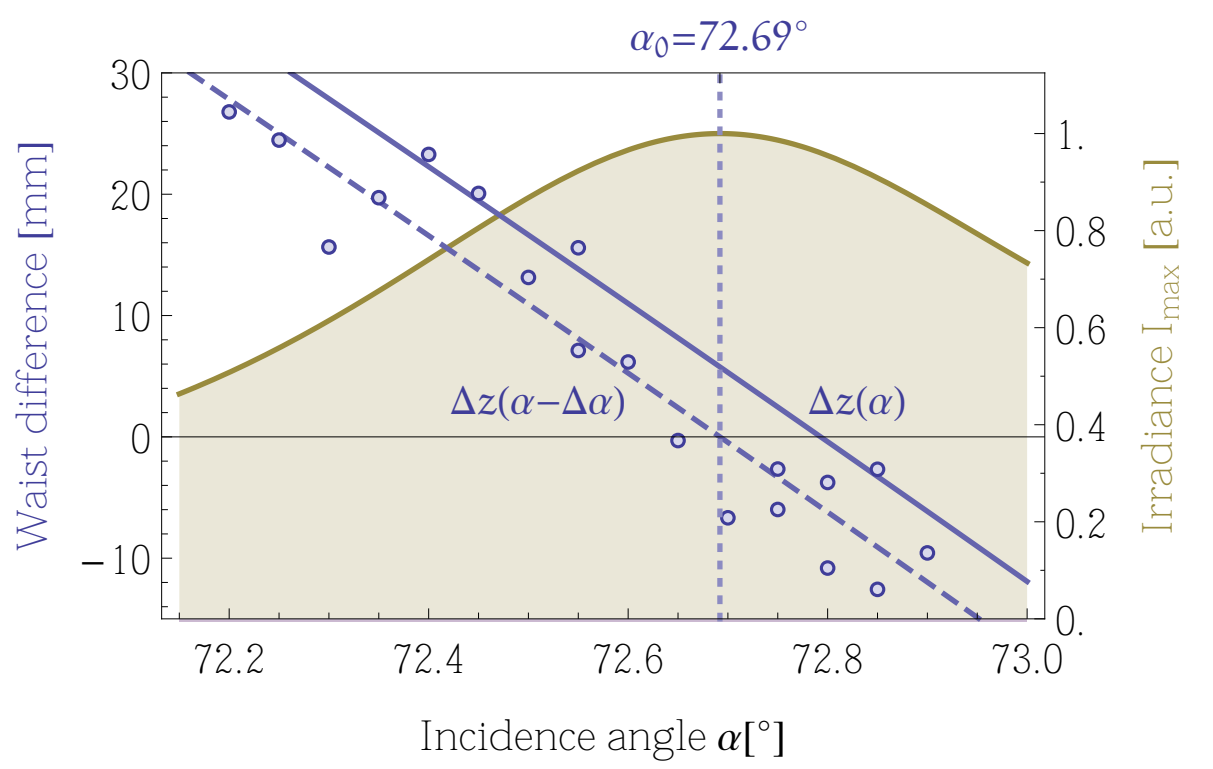

Figure 2.3: Astigmatic waist difference plotted as a function of the angle of incidence. The theoretical curve $\Delta z(\alpha)$ (solid blue line) lies slightly above the experimental values (blue dots). With a shift of $\Delta \alpha=-0.1^{\circ}$, best agreement is obtained (dashed blue line). The achievable irradiance $I_{\max }(\alpha)$ is derived with respect to that coordinate shift.

might contain a small error. A least squares fit routine results a deviation of $\Delta \alpha=-0.1^{\circ}$. The resulting shifted function $\Delta z(\alpha-\Delta \alpha)$ is plotted in figure 2.3 in dashed style revealing good agreement with the measurement. The angle of incidence for a vanishing waist difference is derived to $\alpha_{0}=72.69^{\circ}$. It is expected that for this angle the beam is focused into the smallest beam area. In the following, the achievable intensity depending on the angle of incidence is estimated in arbitrary units.

From matrix $\mathscr{M}(z, \alpha)$, the local beam diameters $d_{x}(z, \alpha)$ and $d_{y}(z, \alpha)$ are derived. Approximating the beam area $A(z, \alpha)=\frac{\pi}{4} d_{x}(z, \alpha) d_{y}(z, \alpha)$ by an ellipse, the mean irradiance at position $z$ is $I(z, \alpha)=P_{0} / A(z, \alpha)$ with an arbitrary beam power $P_{0}$. For a specific angle of incidence $\alpha$, the position $z_{\max }$ is derived which yields the maximum value of $I(z, \alpha)$. This achievable irradiance $I_{\max }(\alpha)$ is depicted together with the astigmatic waist difference in figure 2.3 including the coordinate shift $\Delta \alpha$.

As expected, the highest photon flux is obtained for an angle of incidence of $\alpha_{0}=$ $72.69^{\circ}$, where the astigmatic aberration disappears. It is evident that already a small misalignment of $0.5^{\circ}$ reduces the achievable intensity by $50 \%$ which renders this alignment relevant for imaging applications. Furthermore, phase distortions of the illumination func- 
tion are minimized, which serves to avoid reconstruction errors in case of phase objects.

\section{Conclusion}

With a Hartmann type wavefront sensor, the EUV beam transport is investigated at an HHG setup, where a toroidal grating achieves both spectral filtering and focusing. In an online adjustment, it is demonstrated that already small misalignments of the angle of incidence between EUV beam and toroidal grating lead to significant aberrations and a corresponding loss of intensity. A theoretical approach corroborates the results. For future phase retrieval experiments, this procedure is crucial in order to achieve a flat phase distribution at the sample position, effectively reducing reconstruction errors.

\section{Acknowledgments}

We gratefully acknowledge support by Deutsche Forschungsgemeinschaft within SFB755 'Nanoscale Photonic Imaging' (project C08).

\section{Appendix}

The following ray propagation matrices are employed in this work $[129,130]$ :

$$
\mathscr{S}_{\text {prop }}(z)=\left(\begin{array}{cccc}
1 & 0 & z & 0 \\
0 & 1 & 0 & z \\
0 & 0 & 1 & 0 \\
0 & 0 & 0 & 1
\end{array}\right) \quad ; \quad \mathscr{S}_{\mathrm{tg}}=\left(\begin{array}{cccc}
\frac{\cos \beta}{\cos \alpha} & 0 & 0 & 0 \\
0 & 1 & 0 & 0 \\
-\frac{2}{R_{t}^{\prime}} & 0 & \frac{\cos \alpha}{\cos \beta} & 0 \\
0 & -\frac{2}{R_{s}^{\prime}} & 0 & 1
\end{array}\right)
$$

where, $z$ is a propagation distance, $\alpha$ and $\beta$ are incidence and deflection angle relative to the gratings normal vector, and $R_{t}^{\prime}$ and $R_{s}^{\prime}$ are the effective tangential and sagittal radius, which are defined as

$$
R_{t}^{\prime}=\frac{2 \cos \alpha \cos \beta}{\cos \alpha+\cos \beta} R_{t} \quad ; \quad R_{s}^{\prime}=\frac{2}{\cos \alpha+\cos \beta} R_{s}
$$

with the tangential and sagittal radius $R_{t}$ and $R_{s}$. 
Chapter 2 Toroidal grating astigmatism of high-harmonics... 


\title{
Chapter 3
}

\section{Coherent diffractive imaging beyond the projection approximation: Waveguiding at extreme ultraviolet wavelengths}

Sergey Zayko, Eike Mönnich, Murat Sivis, Dong-Du Mai, Tim Salditt, Sascha Schäfer, and Claus Ropers

Optics Express 23, 19911-19921 (2015).

DOI: 10.1364/OE.23.019911

\begin{abstract}
We study extreme-ultraviolet wave propagation within optically thick nanostructures by means of high-resolution coherent diffractive imaging using high-harmonic radiation. Exit waves from different objects are reconstructed by phase retrieval algorithms, and are shown to be dominated by waveguiding within the sample. The experiments provide a direct visualization of extreme-ultraviolet guided modes, and demonstrate that multiple scattering is a generic feature in extruded nanoscale geometries. The observations are successfully reproduced in numerical and semi-analytical simulations.
\end{abstract}

Classical diffraction theory, within the descriptions of Fresnel, Kirchhoff and Fraunhofer, considers field distributions initially given on a two-dimensional plane [2]. In the most common cases, including those for which closed solutions exist, the initial fields are represented by constant amplitudes on apertures of a compact support. Approximating such conditions experimentally usually involves the illumination of opaque screens with apertures by a collimated beam of light, with a prominent classroom demonstration being Young's double-slit experiment. A frequent implicit assumption is that the screen 
geometrically carves out the aperture shape from the incident beam profile, and that no diffraction occurs within the thickness, i.e., the longitudinal dimensions, of the screen. Mathematically speaking, the scattered light can be represented as a product of the illuminating field and the complex transmission function $\tau(x, y)$ of the aperture. For hard $\mathrm{x}$-rays, in the commonly employed projection approximation, $\tau$ is derived by integrating the optical indices over the object thickness $L$. This is justified as long as, for the wavelength $\lambda$ used, the Fresnel number $F=a^{2} /(\lambda \cdot L)$ for diffraction inside the object remains much larger than unity, where $a$ is a typical transverse length in the object. A related condition on the validity of the factorization approach is given in Ref. [60].

In the case of three-dimensional geometries, wave propagation within the sample may drastically alter the diffraction by an object. For example, surface plasmon polariton contributions may significantly affect double-slit interference [131]. In the presence of optically dense media, multiply scattered waves may experience confinement and waveguiding in the exit wave formation. In particular, at extreme ultraviolet (EUV) wavelengths, such phenomena are technologically important in nanoscale mask design for lithography $[132,133,134,135,136]$. Coherent diffractive imaging (CDI) [67, 6, 112, $117,56,13,66,137]$ provides immediate access to amplitude- and phase-resolved exit wave distributions, and attention has largely been paid to accurate object representation and spatial resolution, including a variety of contrast mechanisms such as magnetism [71] or strain [74]. However, CDI has not yet been used to directly visualize multiple scattering and wave confinement.

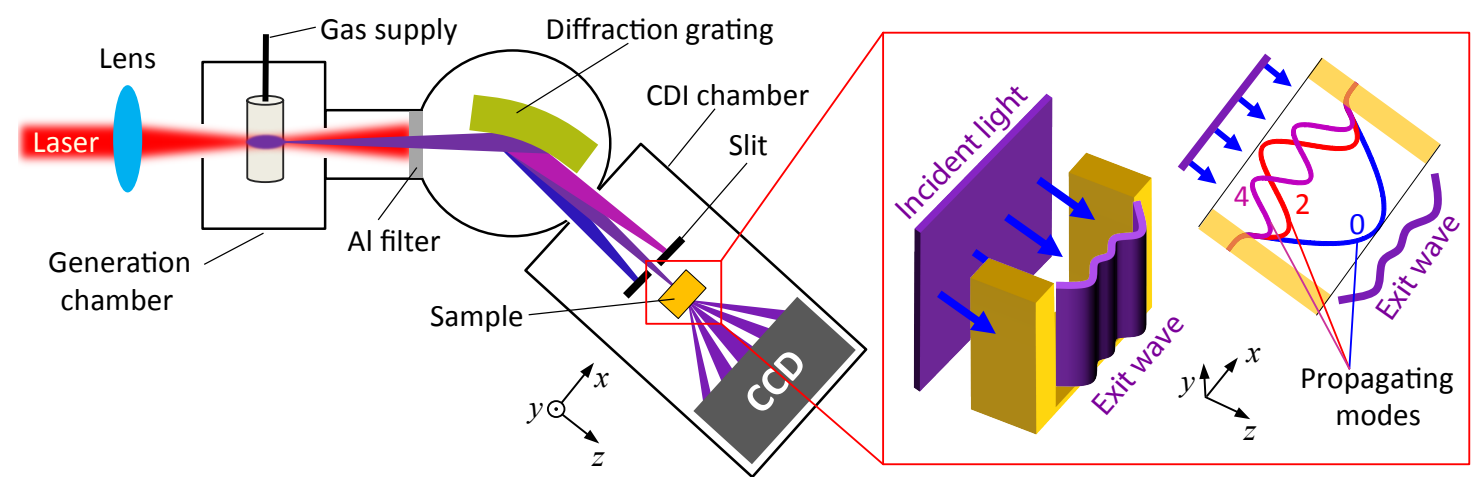

Figure 3.1: Schematic of the experimental setup displaying the high-harmonic generation chamber, the diffraction grating and the imaging chamber (CDI chamber). The inset illustrates the modification of the exit wave induced by propagating waveguide modes within the extended structure (for three even modes denoted by mode indices). 
In this Letter, we utilize CDI with EUV high-harmonic radiation $[12,138]$ to elucidate wave propagation within tailored, optically thick nanostructures. We demonstrate pronounced wave-guiding effects for various geometric parameters, indicating the universal relevance of three-dimensional diffraction phenomena in this spectral range and for typical mask dimensions and aspect ratios. Moreover, we find that for extreme-ultraviolet CDI, spatial mode filtering within thick objects poses a more severe constraint on the achievable spatial resolution than the fundamental limit given by the numerical aperture of detection. The experimental observations are in good agreement with results of numerical finite element modeling and a semi-analytical modal expansion.

CDI experiments are carried out in a high-vacuum setup as schematically depicted in Fig. 3.1. High-harmonic up-conversion of amplified laser pulses (450 $\mu \mathrm{J}$ pulse energy, $40 \mathrm{fs}$ pulse duration, $800 \mathrm{~nm}$ central-wavelength, $1 \mathrm{kHz}$ repetition rate) is induced in an argon-filled capillary, leading to a broad spectrum of discrete harmonics [139]. Details of the generation conditions and EUV photon flux are provided in the Appendix. A $150 \mathrm{~nm}$ thick aluminum foil blocks the fundamental laser beam, transmitting the EUV light with minor losses. The 23rd harmonic order (wavelength $\lambda=34.8 \mathrm{~nm}$ ) in the plateau region of the HHG spectrum is spectrally selected and focused onto the sample by a blazed toroidal grating (550 grooves $/ \mathrm{mm}$ ) and a moveable slit close to the sample position. The use of the diffraction grating serves two purposes: (i) selecting an individual harmonic and separating it from the fundamental beam, and (ii) for objects smaller than a dispersed harmonic in the focal plane, the spatial dispersion of the grating provides for an illumination with enhanced monochromaticity. This allows for an increased number of resolution elements in imaging, i.e., a larger field of view for a given resolution [140, 141]. Coherent diffraction patterns from several non-periodic samples are recorded at distances of $L=20 \mathrm{~mm}$ (object A) and $L=30 \mathrm{~mm}$ (object B) by a cooled, back-illuminated charge-coupled device (CCD) camera (20 $\mu \mathrm{m}$ pixel size, $1340 \times 1300$ array), ensuring sufficient oversampling [59] and a high numerical aperture (NA). The samples are prepared by focused ion beam etching of gold films (460 nm (object A) and $150 \mathrm{~nm}$ (object B) thickness) on $200 \mathrm{~nm}$ silicon nitride membranes. Scanning electron micrographs (SEM) of objects A and B are shown in the insets of Fig. 3.2(b) and 3.2(e), respectively, with dark regions corresponding to aperture areas in the samples.

Figures 3.2(a), 3.2(d) show coherent diffraction patterns of objects A and B. The diffraction intensity and its coherent modulation extend to high numerical apertures, even beyond the detector area for some principal directions. Interestingly, the diffraction patterns 

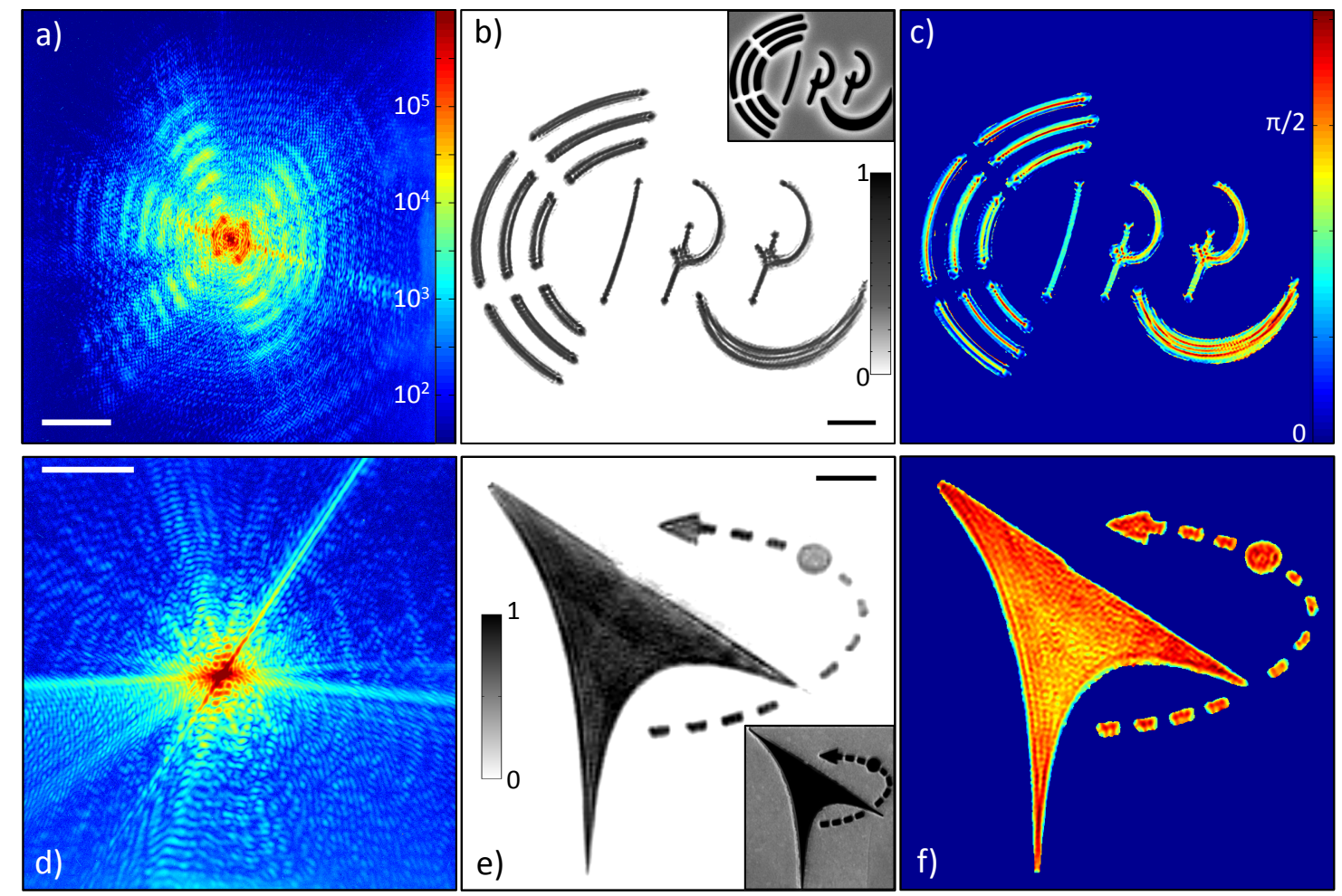

Figure 3.2: (a) Coherent diffraction patterns (a), (d) on a logarithmic scale, reconstructed exit wave amplitudes (b), (e) in arb. units and phases (c), (f) in radians of objects A (top row) and B (bottom row). Insets in (b), (e) display scanning electron micrographs. Scale bars: $5 \mu \mathrm{m}^{-1}$ (a), (d); $1 \mu \mathrm{m}$ (b), (e). Identical colormaps are used for both objects in (a), (d) and (c), (f).

are not centro-symmetric, which implies a non-trivial phase-structure of the exit wave. The images are recorded without blocking the intense central part (no beam stop), so that all spatial frequencies are contained in one acquisition, facilitating exit wave reconstructions (b,e: amplitudes; c,f: phases) by iterative phase retrieval algorithms [4, 142]. We successfully reconstruct the complex-valued exit wave using a variety of different algorithms, with best results obtained with the Relaxed Averaged Alternating Reflections (RAAR) algorithm [52]. A support constraint was generated from the objectâs autocorrelation function (calculated as the inverse Fourier transform of the diffraction pattern) [143]. We apply a phase retrieval transfer function $[144,145,6]$ to estimate the resolution determined by the reconstruction algorithm. The phases are reliably retrieved up to the corners of the detector - above $15 \mu \mathrm{m}^{-1}$, indicating a diffraction limited resolution. Further experimental details concerning image acquisition, processing and reconstruction are given in the Appendix. 
In general, the reconstructed amplitudes and phases represent the distribution of apertures in the otherwise opaque masks, in agreement with the SEM characterization. However, closer inspection reveals significant spatial amplitude and phase modulations, with pronounced dependencies on local aperture width. Fourier truncation artifacts can be ruled out as a cause for these features (see Appendix), and, as we show in the following, these field distributions can be directly attributed to waveguiding effects.

a)

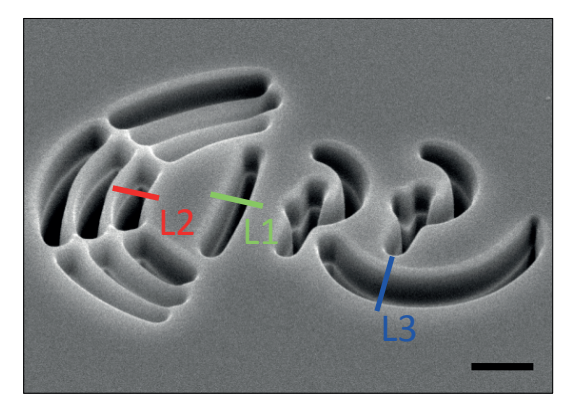

b)

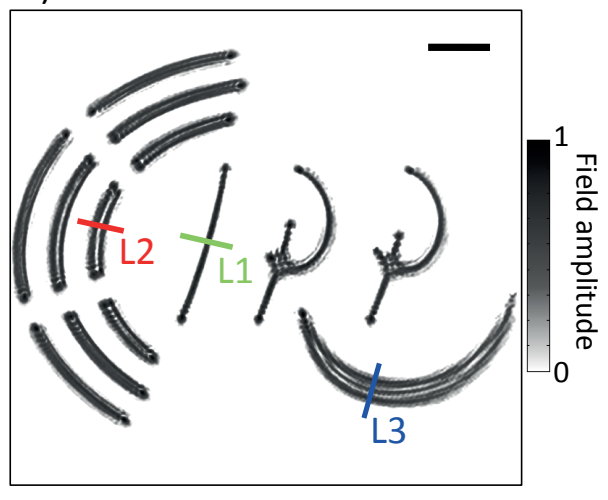

c)
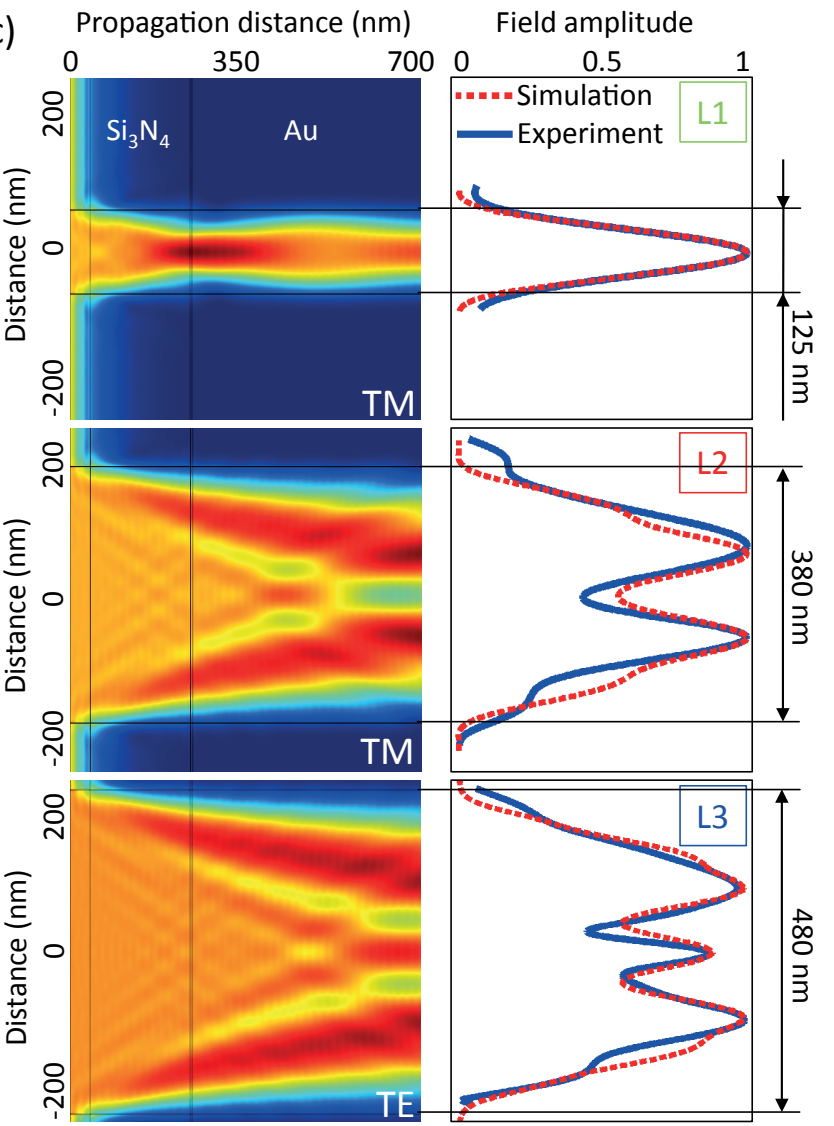

Figure 3.3: (a) Tilted view SEM image of object A. (b) Magnitude of the reconstructed exit field, indicating positions of cross-sections chosen for comparison with wave-guide simulations. (c) Finite element simulations of the wave propagation in slab waveguides of dimensions and materials corresponding to experimental conditions (see text). Left column: Evolution of field magnitudes along the propagation direction for three waveguide widths chosen according to the respective lineouts in (a) and (b). Right column: Comparison of simulated exit field magnitudes (dotted red) with experimental profiles (solid blue). Scale bars are $1 \mu \mathrm{m}$.

Figure 3.3 compares cross-sections through the reconstructed exit wave amplitude with simulated field distributions in planar waveguides of corresponding dimensions. Three 
selected positions for lineouts are indicated in a tilted-view SEM image of object A [Fig. 3.3(a)] and the reconstructed field amplitude [Fig. 3.3(b) $]^{1}$. The cross-sectional shapes of the marked features in the reconstruction vary from a single lobe at line L1 to two and three maxima at lines L2 and L3, respectively. The structure widths at these positions are on the order of 150 (L1), 400 (L2) and $500 \mathrm{~nm}$ (L3), estimated from backside SEM images (see Appendix). With a depth $L$ close to $700 \mathrm{~nm}$, diffraction within the structure is characterized by Fresnel numbers of 0.6, 5.9 and 9.5 respectively. Roughly corresponding to slit-type geometries, these parts of the sample induce an effectively onedimensional confinement for the incident light at a wavelength of $34.8 \mathrm{~nm}$. For the dimensions given, we carry out numerical simulations of the wave propagation within the structure using finite element modeling (COMSOL Multiphysics, Wave Optics Module).

Figure 3.3(c) displays the results of two-dimensional simulations of the electric field propagation along waveguides of different thicknesses corresponding to the positions given in Figs. 3.3(a) and 3.3(b). In the simulations, the incident polarization was chosen to approximate the experimental conditions, i.e., perpendicular to the waveguide slab at positions 1 and 2 (leading to transverse magnetic (TM) modes) and parallel to the slab at position 3 (leading to transverse electric (TE) modes). With the refractive indices of the cladding materials incorporated in the simulations, the computed exit wave amplitudes (Fig. 3.3(c), red dotted lines) closely follows the experimental lineouts (Fig. 3.3(c), blue solid). For simplicity, the small tapering of the waveguides following fabrication (estimated as below 5 degrees half taper angle from SEM characterization) was not included in the simulations, as it is not expected to induce substantial qualitative changes. We obtain very similar results with finite-difference field calculations based on a parabolic wave equation $[146,147]$ and a semi-analytical solution of the waveguide propagation using a modal expansion (see Appendix).

In addition, the modal expansion provides further insight into the waveguiding characteristics in the EUV, where high propagation losses are expected for most cladding materials, e.g., refractive index of gold at $34.8 \mathrm{~nm}: \mathrm{n}=0.77+0.39 \mathrm{i}$ [124]. Figure 3.4 displays real and imaginary parts of the propagation constants $\beta_{n}$, i.e., the wavenumber of mode $n$, for the lowest order TM and TE modes in a gold-cladded vacuum waveguide as a function of waveguide widths, computed by numerically solving the well-known charac-

\footnotetext{
${ }^{1}$ Note that a weak stripe-like contrast in the depth of the structure does not correspond to a step, but arises from chemical contrast from a $4 \mathrm{~nm}$ thick Titanium adhesion layer between the silicon nitride $\left(\mathrm{Si}_{3} \mathrm{~N}_{4}\right)$ and gold layers.
} 


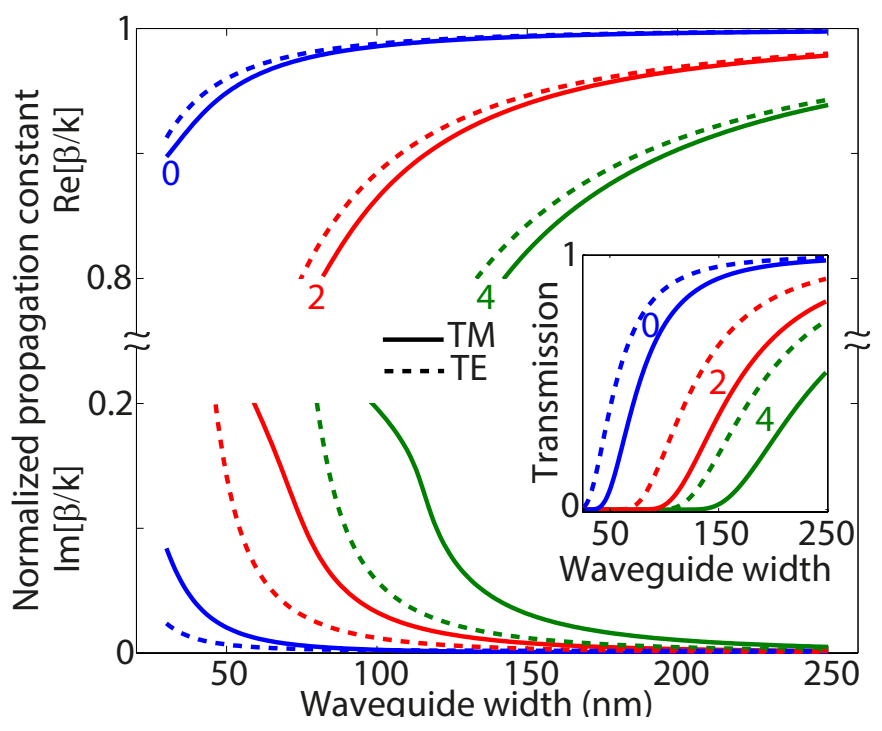

Figure 3.4: Real (top curves) and imaginary (bottom curves) parts of the propagation constant for the first three even TM (solid) and TE (dotted) modes, normalized to the vacuum wave number. Inset: Transmission coefficients through a $700 \mathrm{~nm}$ long gold waveguide of different widths.

teristic equations [148]. Only modes with even indices are shown, since these are the ones excited by far-field radiation under normal incidence. The inset shows the corresponding mode transmission for a waveguide length of $700 \mathrm{~nm}$. In agreement with the simulations in Fig. 3.4, it is apparent that for the smaller features around $100 \mathrm{~nm}$ width, the fundamental mode presents the dominant contribution with a minor additional component from a second even mode. For larger widths, several modes interfere with almost equal amplitude, and the beating of these modes along the waveguide depth determines the exit wave intensity distribution. It is important to note that, due to the enhanced losses of higher order modes, the channels act as effective low-pass filters suppressing high-angle scattering. Therefore, as demonstrated here, the achievable resolution in the imaging of extruded structures may often be limited by waveguiding instead of the numerical aperture of the detection system.

Generally, because of the higher surface reflectivity for light polarization parallel to the waveguide cladding, TE modes exhibit somewhat lower damping and higher transmission. This effect may be used for polarization filtering of the transmitted light. To give a numerical example, in a tradeoff between polarization contrast and TE transmission, polarization contrasts of $10^{2}$ and $10^{3}$ may be obtained at mode transmissions of $15 \%$ and $6 \%$, respectively, for a $300 \mathrm{~nm}$ long waveguide. 
In conclusion, we have presented lensless imaging experiments with a table-top highharmonic source, reconstructing the exit waves of different non-periodic samples. Strong spatial modulations of the field distributions are caused by multiple scattering within the sample, and theoretical modeling accurately reproduces the experimental findings. Besides applications in nanoscale lithography, the phase-resolved imaging of guided modes enables further investigations of waveguiding properties in the EUV spectral range, and is expected to result in the development of new optical devices, such as polarizers, mode filters or wave plates for EUV and soft X-ray radiation.

\section{Appendix A: Sample details}

The samples are prepared by focused ion beam (FIB) etching of $200 \mathrm{~nm}$ thick silicon nitride membranes coated with a layer of gold (thicknesses of $460 \mathrm{~nm}$ and $150 \mathrm{~nm}$ for object $\mathrm{A}$ and $\mathrm{B}$, respectively) on a titanium adhesion layer (4 $\mathrm{nm}$ thickness). In addition, a $40 \mathrm{~nm}$ thick titanium layer is deposited on the opposite side of sample A. The ion beam focusing and current conditions were iteratively optimized to obtain small taper angles in the structures. In the experiment, the samples are illuminated from different sides, as illustrated in Fig. 3.5(a). Sample characterization was carried out by scanning electron microscopy. Exemplary image from the back side of object A is shown in Fig. 3.5(b).
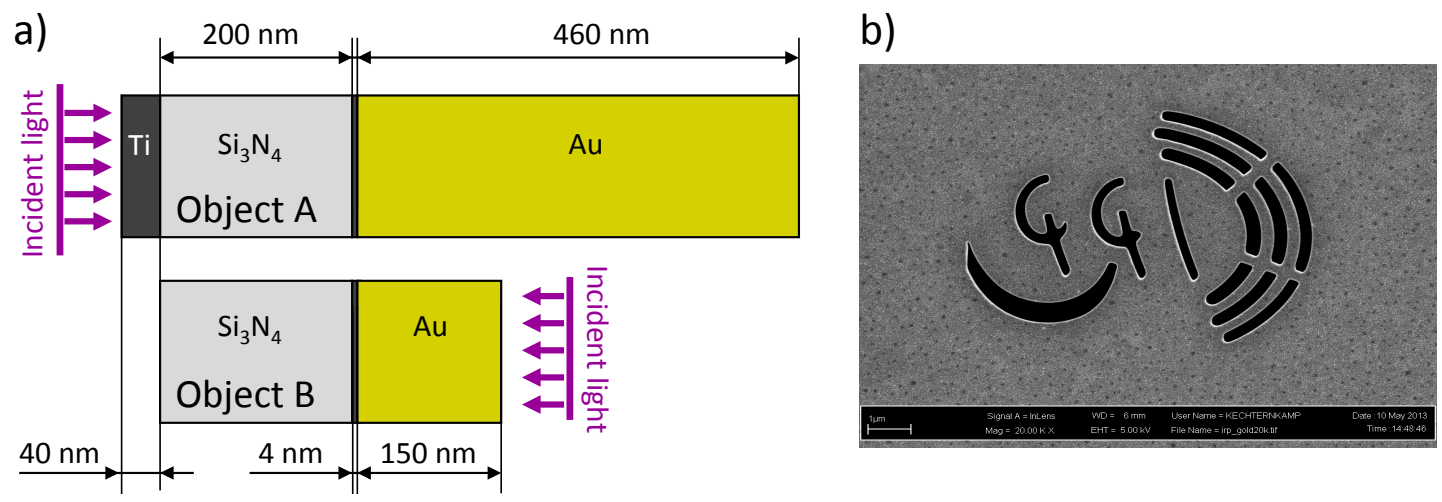

Figure 3.5: (a) Schematic of the layered structure of object A (top) and B (bottom). (b) Scanning electron micrograph of object A, recorded from the back side (gold). Focused ion beam milling was carried out from the front side. Note that the apparent width of the waveguides depends on the SEM focus settings, which gives rise to an uncertainty in the measured widths of several tens of nanometers. 


\section{Appendix B: High-harmonic generation}

For improved conversion efficiency of the generation process, phase-matching is optimized by tuning the gas pressure inside the capillary and the laser focusing conditions. Specifically, the highest yield of the $23 \mathrm{rd}$ harmonic order $(34.8 \mathrm{~nm})$ is obtained at a pressure of $75 \mathrm{mbar}$ and by focusing the laser at the end of the $8 \mathrm{~mm}$ long capillary (focal length $20 \mathrm{~cm}$ ). The measured photon flux (incident on the sample) of the $23 \mathrm{rd}$ harmonic order is $3.4 \cdot 10^{5}$ photons/pulse. The detector was intensity calibrated at the BESSY II, PTB Laboratory.

\section{Appendix C: Reconstruction}

The diffraction pattern of object A [Fig. 3.2(a)] is an accumulation of 200 individual images with $5 \mathrm{~s}$ exposure time each, whereas for object B [Fig. 3.2(d)], we accumulate 300 individual diffraction images of $18 \mathrm{~s}$ exposure time. However, as shown below (Fig. 3.6), even a single acquisition of few seconds integration time is sufficient to obtain a successful reconstruction.

The recorded diffraction patterns were post-processed in several steps: First, dark counts were removed by constant offset subtraction, and the image was centered. Second, the diffraction data was mapped onto a regularly-spaced discrete Fourier domain to account for the use of a flat detector at high numerical apertures (Ewald sphere correction) [78].

Using noise resistance modifications $[149,150]$, we perform reconstructions with the Hybrid Input-Output (HIO) algorithm or the Relaxed Averaged Alternating Reflections (RAAR) algorithm, starting with a random first guess and a real-space support based on the sampleâs autocorrelation derived from the diffraction pattern. To find successful reconstructions within a maximum of 1500 iterations, the real space error in every step is compared to the errors of the two preceding ones. If a local minimum is found, the corresponding reconstruction replaces another one in an archive of 10 reconstructions, given that its error is smaller than the highest error in the archive. By the end of the run, the archive will contain 10 individual reconstructions corresponding to the 10 minima with the lowest errors among all iterations, which can be averaged in a high-quality reconstruction. In this process, the RAAR algorithm displayed somewhat faster convergence. For this extended (autocorrelation-based) support, employing a positivity constraint in 

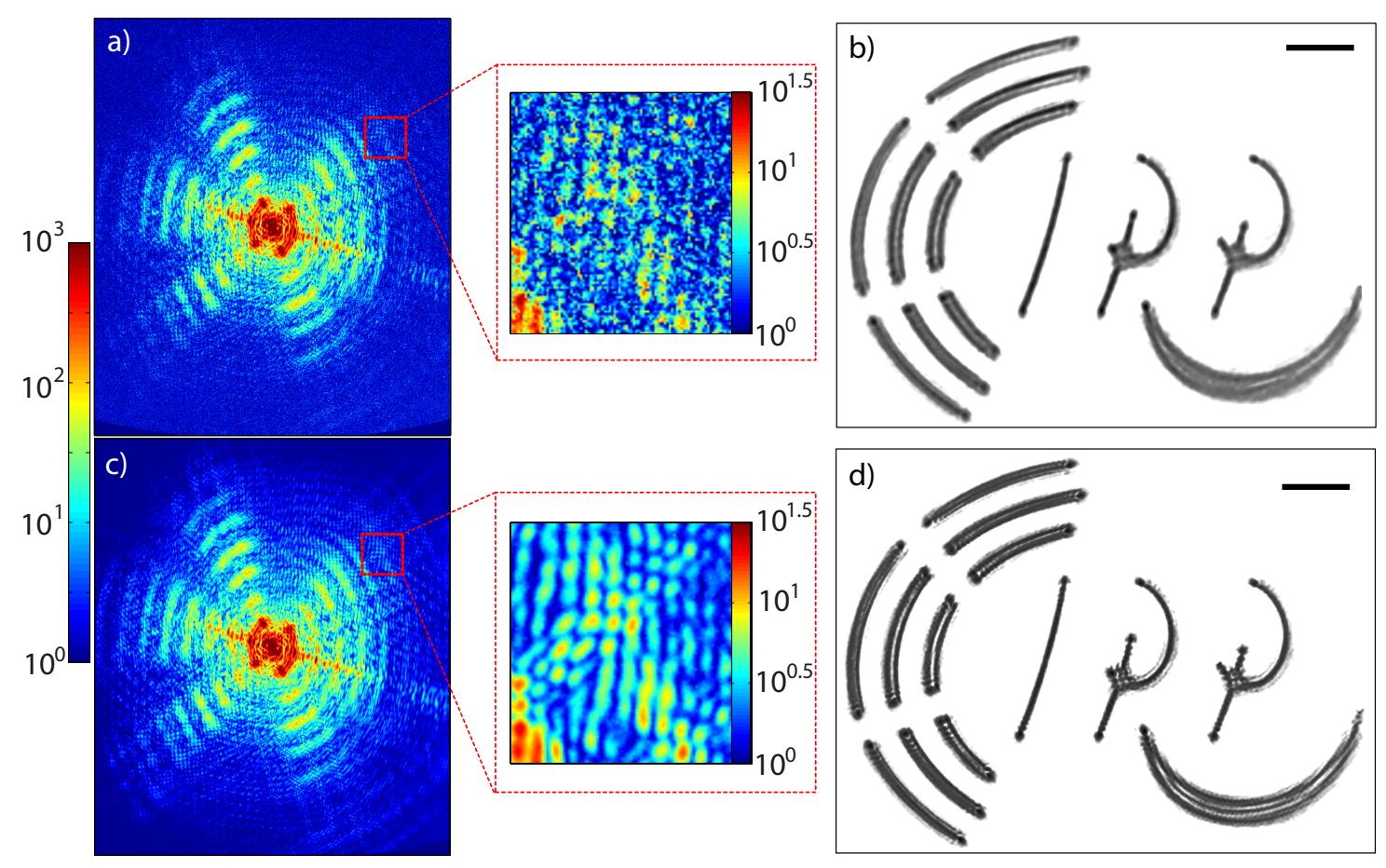

Figure 3.6: (a) Single acquisition (5 s exposure time) diffraction data; logarithmic color scale.

(b) Reconstructed amplitude based on diffraction pattern in (a). (c) Average of 200 individual acquisitions and corresponding reconstruction (d). Scale bars: $1 \mu \mathrm{m}$.

real space (limiting phase variations to less than $\pi$ ) was required to consistently obtain successful reconstructions. However, avoiding the positivity constraint is desirable and physically warranted, and this is achieved with an improved support definition obtained by thresholding the reconstructed amplitudes after a first complete reconstruction. In addition, use of a tighter support accelerates conversion. Reconstructed amplitudes are shown in Fig. 3.2(b), 3.2(e) and Fig. 3.6(d), and phases are shown in Fig. 3.2(c), 3.2(f) for objects A (improved support) and B (autocorrelation support), respectively. Alternatively, the autocorrelation support can be continuously redefined during the first reconstruction run by repeatedly applying a threshold to the current reconstruction after a given number of steps, resulting in a progressive shrinking of the autocorrelation support [151].

Accumulating multiple diffraction patterns improves the signal-to-noise ratio at high diffraction angles and therefore enhances the quality of the reconstruction. Nonetheless, the majority of the object's information is already contained in a single acquisition of few seconds integration time. Figure 3.6 compares the reconstruction using an individual acquisition (single exposure of $5 \mathrm{~s}$, corresponding to 5000 laser pulses) and that from 
averaging 200 individual acquisitions. The latter exhibits improved resolution and detail in the waveguide-induced amplitude modulations.

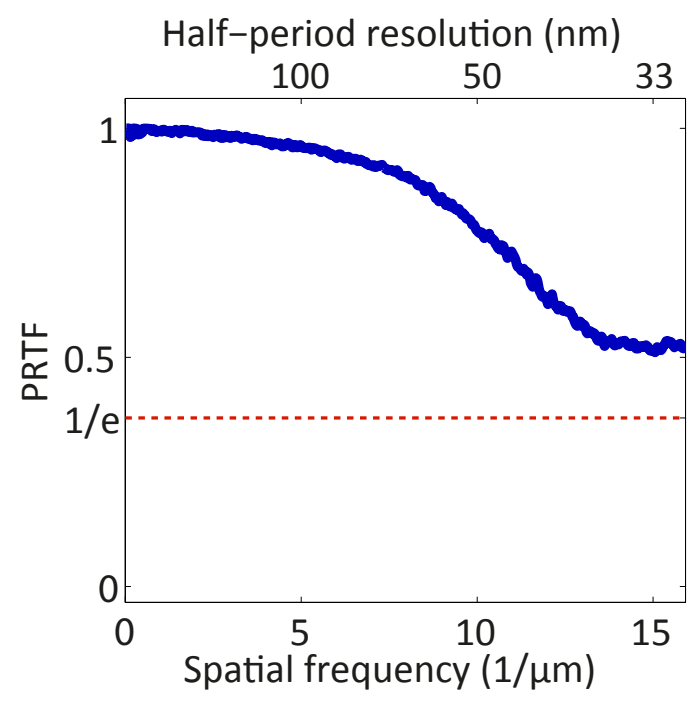

Figure 3.7: Phase retrieval transfer function for the reconstruction shown in Fig. 3.2(b,c).

The resolution limit in the reconstructions is connected to the reliability, with which phases at high spatial frequencies are retrieved, and can be estimated using the so-called phase retrieval transfer function (PRTF) $[144,6]$. In our case, the PRTF evaluates the momentum-dependent consistency of retrieved phases within 30 independent reconstructions, ranging from 1 (full convergence) to 0 (no reliable phase retrieval). Figure 3.7 shows the PRTF plot of the reconstruction of object A. The PRTF values are above 0.5 for all detected spatial frequencies up to $16 \mu \mathrm{m}^{-1}$, corresponding to a half-period resolution of about $31 \mathrm{~nm}$, limited only by the diffraction.

To estimate the resolution we performed 10 individual reconstructions using the RAAR algorithm for 500 iterations with constant relaxation parameter $\beta$ of 0.9999 and autocorrelation support. The reconstruction with the smallest error was chosen to define a new real space support by thresholding to $20 \%$ of the absolute value of the reconstruction. Staring from a random first guess but using the updated real space support another 30 reconstructions were executed with 500 iterations of RAAR. Utilization of redefined support guarantees no flipping of the reconstruction within the support and improves the convergence speed. After first 400 steps in every run, 10 local minima with the lowest errors were averaged and $\beta$ parameter was decreased by 0.2 from 0.999 after every consequent 
25th iteration giving the final reconstruction that was used in the PRTF computation.

\section{Appendix D: Waveguide simulations}

Various theoretical approaches were taken to compute the wave propagation within the structures, including finite element (Fig. 3.2) and finite difference modeling [146]. We approximated the slit-type aperture regions as two-dimensional slab geometries and found this to be a good approximation. The propagation constants and transmission coefficients shown in Fig. 3.2 were computed using a modal expansion for slab waveguides with a cladding of complex refractive index as described in Ref. [148]. The underlying characteristic equations for transverse electric (1) and transverse magnetic (2) modes are:

$$
\begin{gathered}
k_{w} h=2 \arctan ^{-1} \frac{\gamma}{k_{w}}+m \pi \\
k_{w} h=2 \tan ^{-1} \frac{n_{w}^{2} \gamma}{n_{c}^{2} k_{w}}+m \pi
\end{gathered}
$$

Here, $h$ is the waveguide width, $m$ is the mode number, $k_{w}=\left(n_{w}^{2} k_{0}^{2}-\beta^{2}\right)^{1 / 2}$ and $\gamma=\left(\beta^{2}-n_{c}^{2} k_{0}^{2}\right)^{1 / 2}$ represent the transverse wavenumbers in the slab and cladding, where $n_{c}$ and $n_{w}$ are the complex refractive indices of the cladding and waveguide materials (vacuum in our case); $k_{0}=w / c$ is the vacuum wavenumber and $\beta$ is the propagation constant.

\section{Appendix E: Examining Fourier truncations}

In order rule out possible artifacts from Fourier truncation on the spatial amplitude fluctuations in the reconstructions, we simulated the influence of the finite-sized detector. Figure 3.8(a) shows a bitmap image of the object as employed in the focused ion beam fabrication, together with a close-up and a lineout with the ideal binary contrast. In Fig. 3.8(b), the magnitude of the Fourier transform of the object is displayed on a logarithmic scale, and the inner non-shaded part corresponds to the range of Fourier components collected by the detector area (Ewald sphere curvature ignored here for simplicity). Figure 3.8(c) shows the resulting modulations induced by this truncation in the inverse Fourier transformation. The rather weak modulation depth and high uniform spatial frequency are very different from the waveguiding features we have identified in the experiments (Fig. 3.3). 
Therefore, it is clear that truncation artifacts do not contribute in a noticeable manner to the observations.
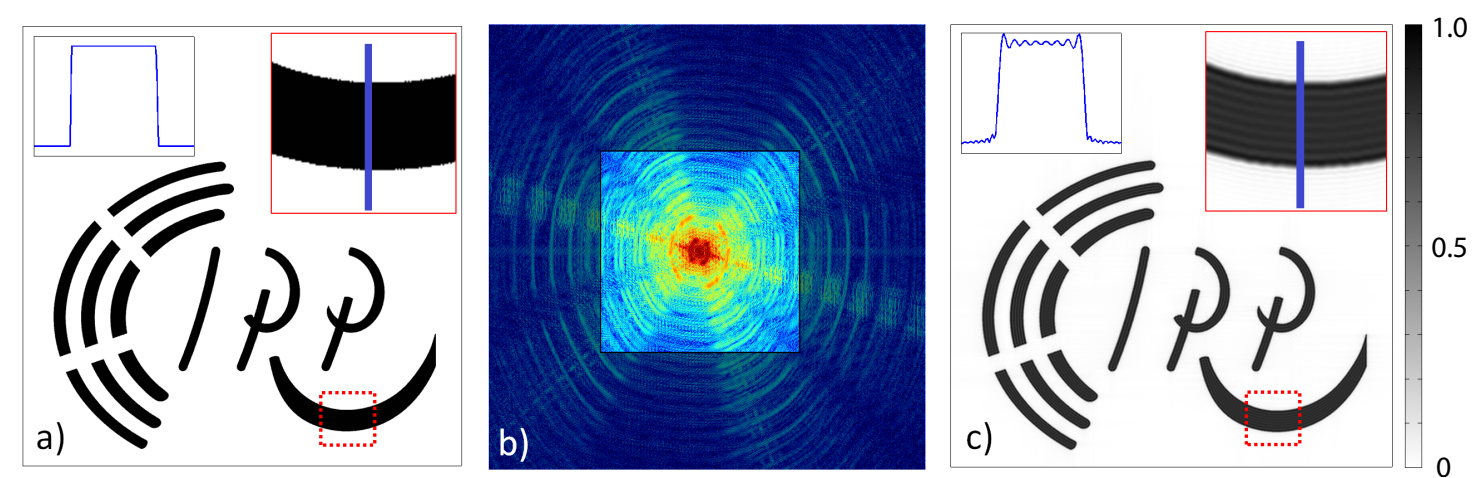

Figure 3.8: (a) Bitmap image of the apertures in object A. Insets show a close-up (dashed red box) and a lineout (blue solid). (b) Magnitude of two-dimensional Fourier transform of the binary object. The inner part corresponds to the range of scattering angles collected by the detector area. (c) Inverse Fourier transform after truncation to the detector area. Minor fluctuations at high spatial frequencies are found. The features are clearly distinct from the waveguiding observed in the experiments (e.g., Fig. 3.3).

\section{Acknowledgments}

We thank Tobias Mey and Klaus Mann for helpful discussions, and Mike Kanbach for sample fabrication. We gratefully acknowledge support from the Deutsche Forschungsgemeinschaft (SFB755 "Nanoscale Photonic Imaging", project C08) and the Triple I consortium within the Action 2 Erasmus Mundus Partnerships. 
Chapter 3 Coherent diffractive imaging beyond the projection approximation ... 


\title{
Chapter 4
}

\section{Polarization contrast of nanoscale waveguides in high harmonic imaging}

Sergey Zayko, Murat Sivis, Sascha Schäfer, and Claus Ropers

Optica, Vol. 3, issue 3, pp. 239-242 (2016)

DOI: 10.1364/OPTICA.3.000239

\begin{abstract}
The optical polarization response of a structured material is one of its most significant properties, carrying information about microscopic anisotropies as well as chiral features and spin orientations. Polarization analysis is therefore a key element of imaging and spectroscopy techniques throughout the entire spectrum. In the case of extreme-ultraviolet (EUV) radiation, however, both the preparation and detection of welldefined polarization states remain challenging. As a result, polarization-sensitive EUV microscopy based on table-top sources has not yet been realized, despite great potentials, for example, in nanoscale magnetic imaging. Here, we demonstrate polarization contrast in coherent diffractive imaging using high harmonic radiation and investigate the polarization properties of nanoscale transmission waveguides. We quantify the achievable polarization extinction ratio for different waveguide geometries and wavelengths. Our results demonstrate the utility of slab waveguides for efficient EUV polarization control and illustrate the importance of considering polarization contrast in the imaging of nanoscale structures.
\end{abstract}

High harmonic generation (HHG), yielding coherent extreme-ultraviolet (EUV) radiation at laboratory scales, has extended the frontiers of ultrafast spectroscopy and imaging to attosecond temporal resolution [21]. Recently, novel schemes leading to efficient HHG 
sources with elliptical $[152,28]$ and circular $[27,30,33]$ polarizations highlighted the potential for studying polarization-sensitive light matter interactions, as demonstrated in HHG-based measurements of circular dichroism [29, 34, 30]. More generally, the polarization state of high harmonic radiation is naturally expected to affect the signal and contrasts in various techniques such as coherent diffractive imaging (CDI) $[13,73,46]$, holography [71] and attosecond spectroscopy [153]. Besides the generation of EUV light with defined polarization, crucial aspects for polarization-dependent imaging and spectroscopy are the control and determination of a given polarization state. Due to the lack of suitable materials exhibiting high optical transmissivity at EUV wavelengths, most approaches used to manipulate and measure polarization in this spectral range rely on reflective components, such as multilayer mirrors or gratings. However, these components are often limited to a rather narrow spectral range, require precise alignment and inevitably influence the direction of light propagation, which imposes additional constraints. Therefore, alternative strategies for polarization control, e.g., in transmission schemes, are highly desirable, and nanostructured materials harnessing EUV waveguiding may be a promising approach $[154,155]$.

Here, we investigate polarization effects in the EUV light propagation through nanoscale slab waveguides by means of HHG-based coherent diffractive imaging, and we discuss the capabilities of these structures to serve as transmission polarizers. Specifically, the light transmitted through the waveguides is analyzed in far-field diffraction, and a phase retrieval algorithm is used to reconstruct the exit wave. We find a strong polarization anisotropy of the waveguide propagation, with enhanced transmission for polarization parallel to the waveguides (TE mode), and observe a polarization contrast that depends on the incident wavelength. A compact linear polarization analyzer is realized by arranging slits with different orientations, mapping the incident polarization into angle-dependent diffraction intensities within a single acquisition. Representing the first demonstration of polarization contrast in lensless imaging using HHG, our results highlight the potential of CDI for the study of locally and microscopically anisotropic and chiral structures.

The schematic of the experimental setup is depicted in Fig. 4.1. A table-top HHG source provides linearly polarized, coherent radiation at odd harmonics of the driving laser frequency (amplified 40-femtosecond Ti:Sapphire laser pulses, $800 \mathrm{~nm}$ central wavelength). Single harmonics in a wavelength range from $28 \mathrm{~nm}$ to $42 \mathrm{~nm}\left(29^{\text {th }}\right.$ to $19^{\text {th }}$ harmonic order) are selected and focused by a combination of a blazed toroidal diffrac- 


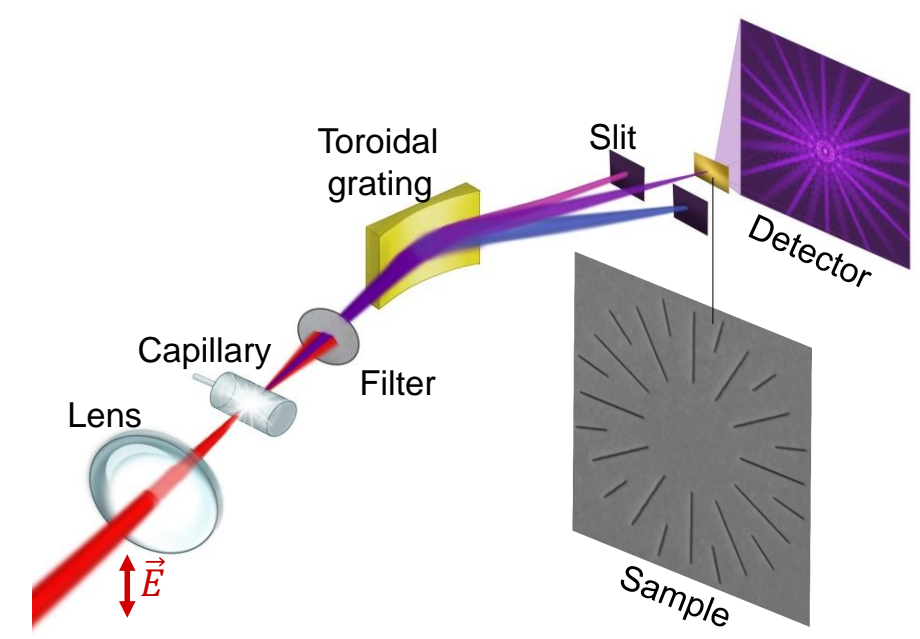

Figure 4.1: Schematic of the experimental setup. High harmonics are generated by focusing femtosecond laser pulses into an argon-filled capillary. After passage through an aluminum filter $(150 \mathrm{~nm})$, a single harmonic is focused onto the sample by a toroidal diffraction grating. A charge-coupled device camera collects diffraction images from waveguide structures.

tion grating and a motorized slit, resulting in quasi-monochromatic, vertically polarized illumination conditions in the sample plane. The sample (see Fig. 4.1) is placed in the EUV focus, and the transmitted light scattered from the nanostructure is collected by a charge-coupled device (CCD) camera at high numerical aperture. The sample orientation is adjusted normal to the HHG beam with a precision better than $0.5^{\circ}$ using the back reflection of an alignment laser. From the recorded far-field diffraction pattern, the exit wave behind the object is reconstructed with diffraction-limited resolution. Further details on the HHG setup and beam characterizations are described elsewhere [154, 156].

The samples are produced by focused ion beam etching of $200 \mathrm{~nm}$ thick silicon nitride $\left(\mathrm{Si}_{3} \mathrm{~N}_{4}\right)$ membranes coated with a $140 \mathrm{~nm}$ gold film. Rectangular slits are milled into the samples, resulting in hollow waveguides with high aspect ratios in both the transverse and longitudinal directions (slit length larger than $1 \mu \mathrm{m}$, slit widths $w$ of few tens of nanometers, depth of $L=340 \mathrm{~nm}$ ). To investigate the polarization dependence of EUV transmission through the waveguides, we designed a pattern with a clock-like arrangement of $50 \mathrm{~nm}$ wide slits, as shown in the scanning electron microscope (SEM) image in Fig. 4.2(a). Employing linear incident polarization [vertical in Fig. 4.2(c)], within a single diffraction pattern, different slit orientations yield direct access to the transmission intensity as a function of the angle between the polarization axis and the waveguides. Due to 
the high EUV absorption of the materials used, the transmission through the non-etched regions is below $10^{-10}$, such that only waveguide transmission contributes to the signal.
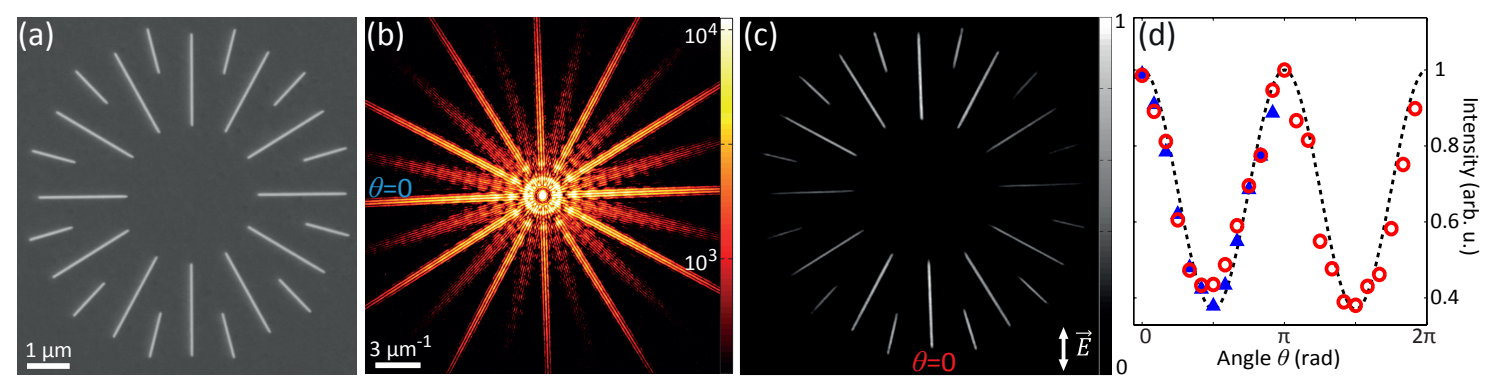

Figure 4.2: Demonstration of polarization contrast in CDI. (a) Scanning electron micrograph of a sample carrying a clock-like arrangement of slab waveguides. (b) Recorded diffraction pattern at $35 \mathrm{~nm}$ wavelength. (c) Reconstructed exit wave intensity obtained by iterative phase retrieval. The angle-dependent intensity variation reflects the polarization contrast in transmission (incident polarization: vertical). (d) Transmitted intensity plotted as a function of the slit orientation angle $\theta$ [ $\theta=0$ corresponds to the vertical waveguide in the bottom of (c)]. Red circles: Intensity evaluated from the exit wave reconstruction. Blue triangles: Intensity obtained directly from the diffraction pattern. Dashed line: Fit to the behavior of an imperfect polarizer (see text).

Figure 4.2(b) shows the far-field diffraction intensity (logarithmic scale), recorded at a distance of $25 \mathrm{~mm}$ behind the sample and for illumination with a wavelength of $35 \mathrm{~nm}$ ( $23^{\text {rd }}$ harmonic). Sufficient oversampling [46] in the diffraction pattern allows for a reconstruction of the field amplitudes and phases of the exit wave. We use the relaxed averaged alternating reflection (RAAR) algorithm [52] and apply it to the diffraction pattern together with an autocorrelation-based real space support, obtained from the inverse Fourier transform of the diffraction pattern. Figure 4.2(c) displays the reconstructed exit wave intensity, which is in close agreement with the overall shape of the structure. The objects studied are significantly smaller than both the focus diameter and lateral wavefront distortions, as determined from previous beam characterizations [156]. Therefore, the current conditions essentially correspond to plane wave illumination, and the reconstructed exit wave represents the complex transmission functions of each individual waveguide in a single reconstructed image. As apparent from Fig. 4.2(c), the reconstructed exit wave intensities are strongly dependent on the slit orientation. Specifically, vertical waveguides, i.e., those parallel to the incident polarization, are noticeably brighter than horizontal ones. The same behavior is evident also in the diffraction pattern, taking into account that the far-field is composed of a superposition of diffracted exit wave fields from pairs of 
elongated rectangular apertures. Each of the slit pairs contributes a diffraction streak in the respective perpendicular direction. Specifically, following standard diffraction theory [157], the extended length of the diffraction streaks in reciprocal space is governed by the strong confinement from the narrow waveguide width, the widths of the streaks are determined by the length of the individual slits, and double-slit interference between slits of the same orientation cause a fine sinusoidal modulation of the streaks. At high spatial frequencies, the streaks from differently oriented slits are particularly well-separated.

In Figure 4.2(d), the integrated exit wave (red circles) and far-field (blue triangles) intensities (normalized to the length of the individual waveguides) in the reconstruction and the diffraction pattern, respectively, are plotted as a function of the slit orientation angle $\theta$. Here, $\theta$ is defined as ranging from 0 to $2 \pi$, corresponding to the full circle of slits. The high reproducibility of the fabrication is evident from the nearly identical signals measured for each of the four equivalent slit orientations with equal $\bmod (|\theta|, \pi)$, e.g., $\theta= \pm n \cdot 15^{\circ}$ and $\theta=180^{\circ} \pm n \cdot 15^{\circ}$ with integer $n$. Because of the inversion symmetry of the diffraction streaks in Fig. 4.2(b), both opposing parts of each feature are combined, such that the blue symbols in Fig. 4.2(d) are only plotted from 0 to $\pi$. The normalized experimental data points evaluated from the diffraction pattern and the reconstruction agree with the expectation of an imperfect polarizer [Malus law, black dashed line in Fig. 4.2(d)]: $I_{t r}(\theta)=\cos ^{2} \theta+C \cdot \sin ^{2} \theta$, where $C=I_{\min } / I_{\max }=1: 2.7$ is the polarization extinction ratio between maximum and minimum transmission.
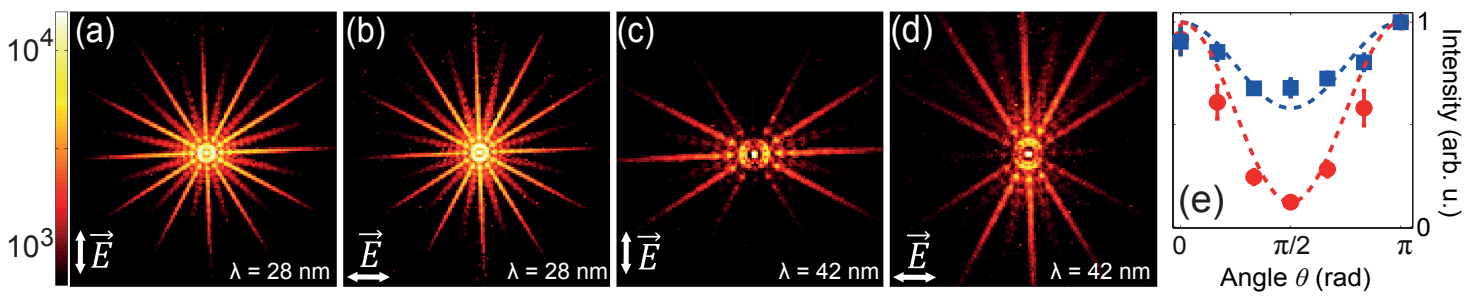

Figure 4.3: Wavelength-dependence of polarization contrast. (a)-(d) Far-field diffraction images of the object from Fig. 4.2 for different polarization angles [(a),(c): vertical; (b),(d): horizontal)] and wavelengths $\lambda$. (e) Transmission as a function of waveguide orientation $\theta$ (evaluated for vertical polarization) at $\lambda=28 \mathrm{~nm}$ (blue) and $42 \mathrm{~nm}$ (red), showing increased contrast at longer wavelength.

The achievable extinction ratio will depend on the illuminating wavelength, as well as the waveguide properties, such as the width, propagation length and the cladding materials. In order to study such dependencies and identify conditions with higher contrast, we have 
performed measurements using different illumination wavelengths and narrower waveguides. As the converged exit wave reconstruction and the diffraction data yielded the same polarization contrast, in the following, we limit the discussion to direct evaluations of the diffraction data.

Figures 4.3(a)-(d) show a set of far-field diffraction patterns obtained for vertical and horizontal incident polarizations (indicated by arrows) and at illumination wavelengths of $28 \mathrm{~nm}(\mathrm{a}, \mathrm{b})$ and $42 \mathrm{~nm}(\mathrm{c}, \mathrm{d})$. The normalized transmitted intensity is plotted in Fig. 4.3(e) as a function of $\theta$ [evaluated from Figs. 4.3(a) and 4.3(c)], displaying a strong increase of polarization contrast with wavelength, which reaches 1:1.5 and 1:8 at wavelengths of $28 \mathrm{~nm}$ and $42 \mathrm{~nm}$, respectively.

We investigated the influence of the waveguide width on the extinction ratio by using a second structure composed of narrower waveguides [cf. SEM image in Fig. 4.4(a)]. The pattern contains $40 \mathrm{~nm}$ wide waveguides (estimated from SEM characterization) with only two perpendicular slit orientations. This configuration is chosen to increase the transmittance of the structure and allows for an evaluation of the polarization contrast with improved signal-to-noise ratio. Figure 4.4(b) depicts a diffraction pattern (logarithmic intensity scale) obtained with $\lambda=38 \mathrm{~nm}$ radiation and vertical incident polarization. A high polarization contrast of $C=1:(28 \pm 1)$ is found, with maximum diffraction intensity again stemming from vertical slits (corresponding to TE mode transmission, in which the electric field is parallel to the waveguide walls). For comparison, Fig. 4.4(c) displays a diffraction pattern recorded after rotation of the polarization of the HHG pump beam by $30^{\circ}$, which (after grating reflection) results in a weakly elliptical polarization (ellipse indicated) and, therefore, increased diffraction from the horizontal waveguides.

In order to quantitatively model our results, we theoretically treat the light propagation through the nanoscale waveguides within a modal expansion. Specifically, we solve Maxwellâs equations for transverse electric (TE) and transverse magnetic (TM) modes in gold- and $\mathrm{Si}_{3} \mathrm{~N}_{4}$-coated, vacuum-core slab waveguides, obtaining complex propagation constants $\beta_{T E / T M}(\lambda, L)$ as a function of wavelength and waveguide width. For the aspect ratios given (depth vs. width), the transmission is governed primarily by the fundamental mode, while higher order modes are absorbed [154]. Assuming equal incoupling to the waveguide by both polarizations, the polarization extinction ratio is determined by the imaginary parts of the propagation constants and the waveguide thickness: $C=\exp \left(-2 \cdot \operatorname{Im}\left(\beta_{T M}-\beta_{T E}\right) \cdot L\right)$. The origin of higher TE transmission compared to TM transmission, something previously found at longer UV wavelengths $[155,158]$, should 

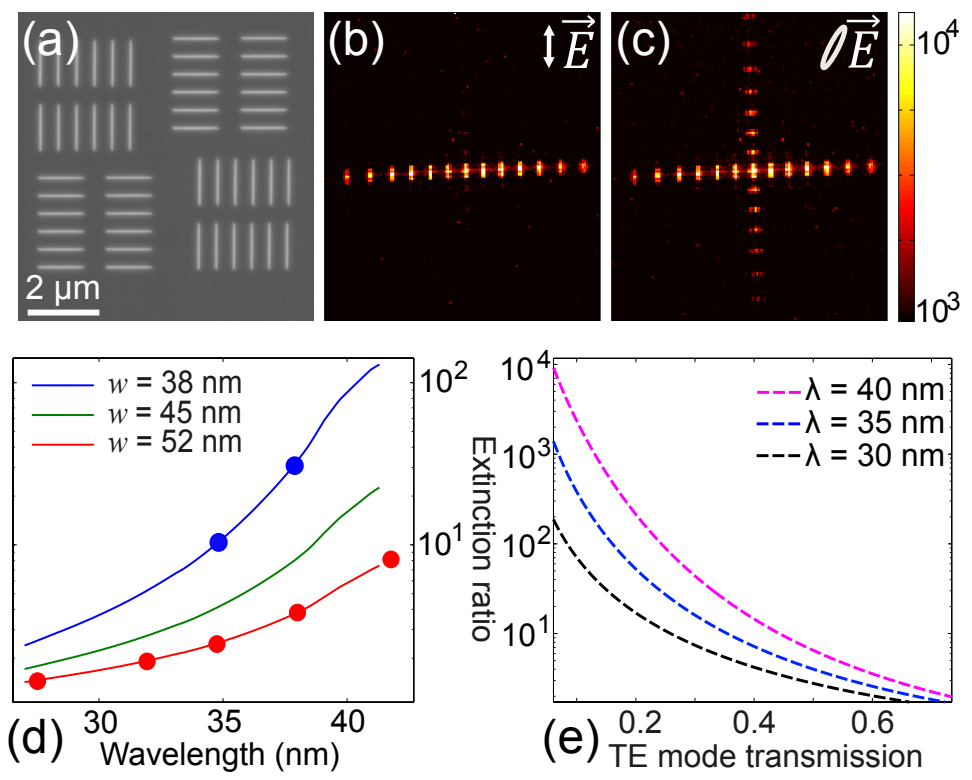

Figure 4.4: (a) SEM image of a structure with two orthogonal slit orientations. (b,c) Diffraction pattern recorded at $\lambda=38 \mathrm{~nm}$ for vertical (c) and elliptical (d) polarization, indicated by the tilted ellipse (see text). (d) Solid lines: Simulated extinction ratios as a function of $\lambda$ for different slit widths $w$ in the double-layer geometry of the experiments. Circles: Experimentally determined extinction ratios for the structures in Fig. 4.2 (red) and Fig. 4.3 (blue). (e) Simulated polarization contrast of a $300 \mathrm{~nm}$ deep gold waveguide as a function of TE mode transmission.

be discussed. This observation is opposite to what is widely found in metallic wiregrid polarizers at visible and infrared wavelengths [157]. These devices employ the fact that there is a cutoff for TE polarization (but not for TM polarization) at sub-wavelength widths and for high-conductivity cladding. At EUV wavelengths, on the other hand, the materials used exhibit high absorption. Thus, the enhanced Fresnel reflection coefficients of TE compared to TM polarization at the waveguide walls is responsible for the polarization contrast. Narrower waveguides involve higher effective propagation angles [157], such that high contrasts are achieved even for waveguides wider than the wavelength.

For our waveguides composed of two materials (200 nm of $\mathrm{Si}_{3} \mathrm{~N}_{4}$ and $140 \mathrm{~nm}$ of gold), the propagation constants were individually computed. In the case of perfect mode overlap at the transition from gold to $\mathrm{Si}_{3} \mathrm{~N}_{4}$ cladding, the overall polarization contrast follows as the product from both layers. Finite element calculations ${ }^{1}$ showed this to be a good approximation for our geometries, and were also in quantitative agreement with the modal

${ }^{1}$ COMSOL Multiphysics, Wave Optics Module. 
expansion regarding total transmission and polarization extinction ratio. Figure 4.4(d) plots the polarization extinction ratio of the two structures investigated (circles) as a function of illuminating wavelength in comparison with the simulation results for different waveguide widths. The curves illustrate the strong increase in contrast for longer wavelengths and narrower waveguides. Very good agreement between the experimental and simulated extinction ratios is found for widths of $52 \mathrm{~nm}$ (pattern in Figs. 4.2-4.3) and $38 \mathrm{~nm}$ (pattern in Fig. 4.4), which is consistent with the SEM width characterization.

The increase of polarization contrast with wavelength is caused by both higher absorption losses for stronger mode confinement, and more importantly in the case of gold, the wavelength dependence of the refractive index. For a fixed wavelength, the polarization contrast can be further increased at the expense of total transmission by tuning the waveguide width and depth. Figure 4.4(e) shows the simulated polarization contrast of $300 \mathrm{~nm}$ long gold waveguides for different illuminating wavelengths as a function of the TE mode transmission that results from varying the waveguide width. The simulations highlight the potential for polarization control in the EUV spectral range with waveguide-based structures. For example, at a wavelength of $40 \mathrm{~nm}$, the polarization contrast exceeds $30 \mathrm{~dB}$ at a TE mode transmission of 0.15 (corresponding to a $37 \mathrm{~nm}$ waveguide width). Large-area structures with similar aspect ratios can be readily produced using state-of-the art nano lithography and chemical etching [159]. Such structures present a compact alternative to existing schemes for HHG polarization characterization [152, 34, 160]. Combined with reflective waveplates, this approach may also yield the complete state of polarization. We note that both the overall waveguide transmission and in particular the extinction ratios are weak functions of incidence angle, rendering these structures suitable for applications with beam tilts or divergences of at least 10 degrees.

In conclusion, we investigated the polarization-dependent EUV light propagation in nanometric slab waveguides using HHG-based lensless imaging and far-field diffraction. The experimentally measured polarization extinction ratios were in close agreement to theoretical predictions, evidencing the suitability of waveguide arrays in future polarization-sensitive imaging and spectroscopy applications. More generally, our results demonstrate the feasibility of polarization contrast microscopy in the extreme ultraviolet using laboratory scale sources. The further development of birefringent transmission waveguides may enable comprehensive polarization control for applications such as magnetic and chiral imaging. 


\section{Funding Information}

We acknowledge funding by the Deutsche Forschungsgemeinschaft (SFB755, project C8).

\section{Acknowledgments}

We gratefully acknowledge discussions with Tim Salditt. 
Chapter 4 Polarization contrast of nanoscale waveguides ... 


\section{Chapter 5}

\section{General discussion}

In this Chapter, the main results of the preceding studies are summarized and discussed. Additionally, future prospects of the developed HHG source as well as EUV optical elements based on nanoscale waveguides are provided.

\subsection{High-resolution coherent diffractive imaging}

In spectral ranges, where a development of optical elements is infeasible, lensless imaging techniques offers the possibility of performing photon-efficient imaging experiments with the resolution limited only by diffraction (cf. Eq. 1.1). Among these techniques, coherent diffractive imaging is, perhaps, the most versatile and promising method. For example, Fourier transform holography strongly relies on the interference of scattered light with a reference beam, which limits the resolution and the contrast of reconstructions. Ptychography offers, in principle, an unlimited field of view and may improve the convergence of the reconstruction algorithm, but the scanning scheme diminishes the ability to perform single shot or non-repetitive measurements. In addition to that, this technique induces dependencies on the source stability as well as the sample positioning precision. In contrast, CDI relies solely on a single diffraction pattern, recorded with a known wavelength at a known distance. A small beam shifts of the probe or sample vibrations influence only the phase of the scattered light in the far-field and does not change the intensity distribution, if the sample is homogeneously illuminated. Nevertheless, certain experimental requirements have to be met in order to use coherent diffractive imaging.

The applicability of CDI strongly depends on the probe parameters as well as the object's properties. The first includes spatial and temporal coherence and wavefront charac- 
teristics at the sample position. For instance, the numerical fast Fourier transform (FFT), used in phase retrieval algorithms to approximate the far-field diffraction of the scattered exit wave, is valid only for a purely monochromatic beam exhibiting a perfect spatial coherence. Any deviations from this assumption will strongly influence the validity of the FFT and, thereby, may reduce the convergence probability of a reconstruction algorithm. The spatial coherence ensures the appearance of interference fringes. Thorough investigations of the coherence requirements for CDI reveal that the spatial coherence must be at least two times larger than the lateral size of the object investigated [140]. This requirement can be mitigated if a slightly curved wavefront is used for illumination $[161,162]$. The temporal coherence of a source with spectral bandwidth $\Delta \lambda$ is related to the monochromaticity of the beam with a central wavelength $\lambda$, as $\lambda^{2} / \Delta \lambda$. Photons with higher energies within $\Delta \lambda$ will have the same momentum transfer at smaller scattering angles and, thus, appear at different positions in the diffraction pattern - namely closer to the middle. The broadening of the scattering angles reduces the sharpness of the interference fringes, and inevitably reduces the reliability of the phase retrieval algorithm, thereby reducing the resolution of the reconstructed image [115, 141], which is $R_{h p}>\frac{O D}{\lambda / \Delta \lambda}$ [59]. Here, $O$ is the oversampling ratio and $D$ is the sample size. In some cases, precise ab-initio knowledge of the probe spectrum can be implemented into the reconstruction algorithm, wherein the measured diffraction pattern is treated as a superposition of all individual spectral components. This allows for polychromatic CDI with quasi-discrete spectra [115, 163] and even broadband sources [116, 164]. Polychromatic CDI can be beneficial for the simultaneous study of structures exhibiting several absorption or resonance edges within a single specimen. However, despite a possible gain in the total flux, the achieved resolution of such reconstructions is typically lower compared to the conventional monochromatic CDI scheme.

In contrast to commonly used techniques for monochromatization of high harmonic radiation using multilayer EUV mirrors [112, 114, 13, 92, 117, 56, 137], our experimental configuration utilizes a toroidal diffraction grating. Despite the complicated alignment procedure and a slightly weaker reflection efficiency compared to a single state-of-theart EUV mirror, a diffraction grating has three important advantages regarding nanoscale lensless imaging:

1) Wavelength tuning is easily realized by rotating the grating to an appropriate incident angle, whereas multilayer mirrors are optimized for a certain, relatively narrow band.

2) The monochromaticity of the EUV beam impinging on the micrometer-scale structure 
is sufficiently improved, compared to that of two sequential multilayer mirrors with typical values of $\lambda / \Delta \lambda$ up to $200-250[92,112]$. The linear dispersion of the grating used in this work at the focal spot is $\sim 2 \mathrm{~nm} / \mathrm{mm}(0.002 \mathrm{~nm} / \mu \mathrm{m})$. Thus, the spectral bandwidth incident onto the microscopic object is very narrow and $\lambda / \Delta \lambda$ can easily exceed magnitudes of several thousands when the lateral dimension of the sample is few $\mu \mathrm{m}$ improving the spatial resolution of the reconstructed images. Note that, the spectral bandwidth incident onto the sample also depends on the resolving power of the grating, i.e., the number of the slits illuminated.

3) A set of multilayer mirrors cannot prevent the overlap of harmonics of different orders and the residual fundamental beam, which drastically reduces the monochromaticity of the probe. Thus, the utilization of the diffraction grating in our HHG setup is beneficial for high-resolution CDI.

Using this formula $R_{h p}>\frac{O D}{\lambda / \Delta \lambda}$ and the oversampling requirement $(O>5)$ provided by Miao et al. [67], one can compute the expected spatial resolution available by highharmonic imaging employing multilayer mirrors for monochromatization of the EUV radiation. Clearly, the spectral bandwidth $\lambda / \Delta \lambda$ accessible by a set of two multilayer mirrors (typically ranging between 200 and 250) is not sufficient to perform imaging with resolution better than $40 \mathrm{~nm}$ even for a relatively small field of view of $2 \mu \mathrm{m}$. Thus utilization of a diffraction grating is a key aspect for harmonic imaging with high spatial resolution.

Unlike in the case of X-rays, where diffraction occurs at very small angles, EUV radiation scatters at relatively large angles that have to be detected in order to achieve high spatial resolution. In our experiments, the numerical aperture of the detected signal in some cases exceeds the value of 0.55 , corresponding to a maximum scattering angle of $34^{\circ}$. In this case, the wavefront curvature of the scattered light is essential, and aberrations, resulting from the detection with a flat CCD camera, must be corrected. Curvature distortions influence not only the quality of the reconstruction, but also strongly affect the convergence of the phase retrieval algorithm. Figure 5.1 compares the diffraction patterns and the central parts of the corresponding reconstructions, obtained with [Fig. 5.1(b,d)] and without [Fig. 5.1(a,c)] a curvature correction of the diffraction pattern, respectfully, recorded at $2.5 \mathrm{~cm}$ distance $(N A=0.47)$ after illumination of sample A using the 23rd harmonic and 5 seconds exposure time. Figure 5.1(a) shows the measured data, whereas Fig. 5.1(b) depicts the same data, plotted onto the Ewald sphere to account for aberrations caused by projection distortions. For both reconstructions [Fig. 5.1(c) and Fig. 5.1(d)], 


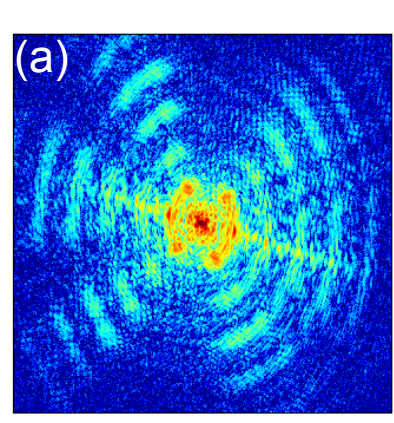

(c)

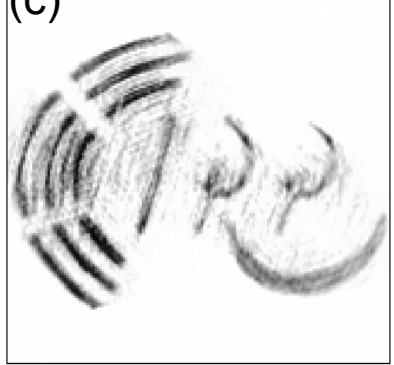

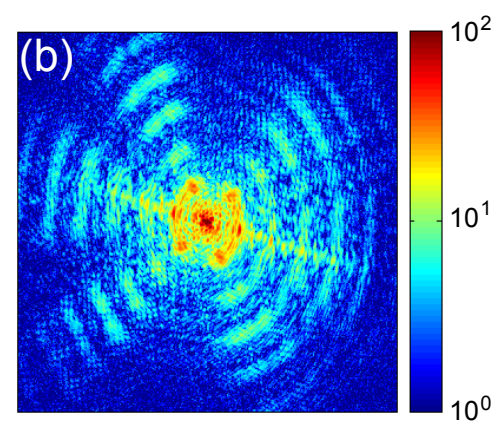

(d)

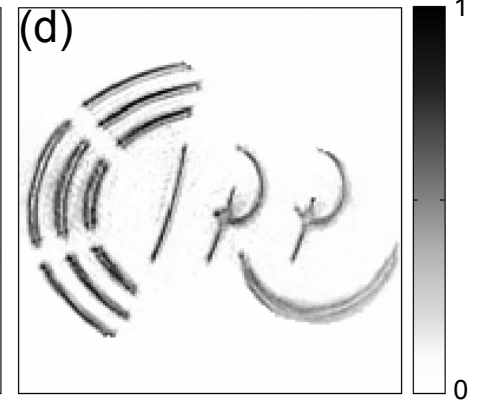

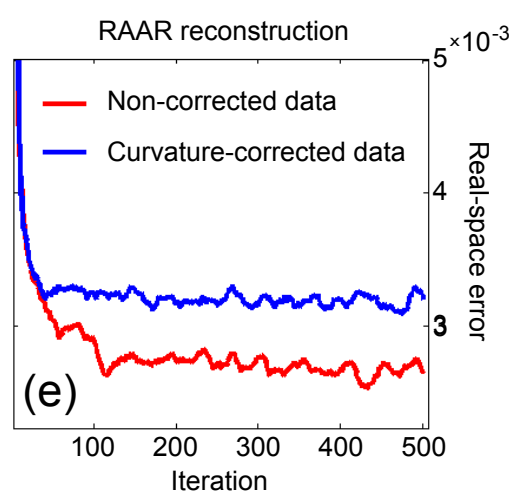

Figure 5.1: Spherical corrections of diffraction data with high numerical aperture. (a) Diffraction pattern recorded at $2.5 \mathrm{~cm}$ distance between the sample and detector and (c) is the corresponding reconstruction. (b,d) Diffraction pattern and the corresponding reconstruction of the same experimental data, but using the spherical corrections for measurements recorded at high NA. (e) Evolution of the real-space error during the reconstruction process.

identical algorithm parameters and real space supports from the autocorrelation of the sample (inverse Fourier transform of the measured far-field intensity with applied low pass filter) were used. However, there is a noticeable difference between the reconstruction results. This is also evident in the improvement of the real space error shown in Fig. 5.1(e), indicating a better convergence of the phase retrieval algorithm. Unlike the curvature corrected data, the non-corrected diffraction pattern cannot be reconstructed using the autocorrelation support without breaking the symmetry ${ }^{1}$. The closer the distance between the sample and the detector, the stronger the distortion and, therefore, the mismatch between the measured diffraction data and the numerical fast Fourier transform of the scattered exit wave is larger. For the experimental data recorded at $2 \mathrm{~cm}$ distance, curvature distortions become too strong and totally diminish the convergence of the algorithm for the non-corrected data, even if a very tight real space support is used.

The ultimate goal of the preparation of experimental data for reconstruction is to min-

\footnotetext{
${ }^{1}$ A non-centrosymmetric real space support prevents flipping of the reconstruction algorithm between two possible solutions that are complex conjugate of each other.
} 
imize the mismatch between the experimentally measured far-field diffraction and a numerical Fourier transform of the scattered exit wave. This mismatch can be caused by several phenomena, including low coherence of the probe, aberrations associated with the curvature of the scattered wavefront measured by a flat detector, readout noise and the dark current of a the CCD chip, cosmic rays, etc. When the aforementioned experimental challenges are considered and accounted for, a phase retrieval algorithm can provide high quality reconstructions with acceptable convergence and a diffraction-limited resolution. As described in Chapter 1, the numerical aperture $N A$ and the real space pixel
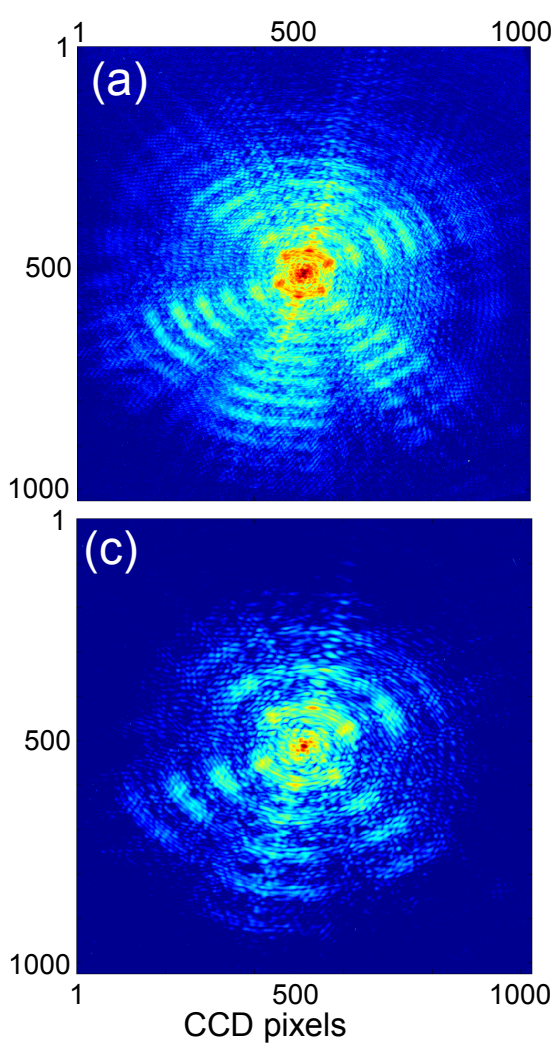

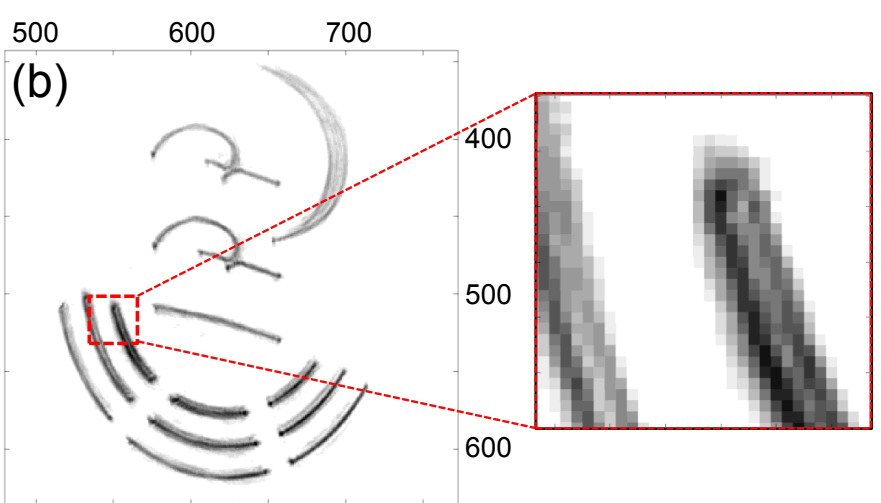

(d)

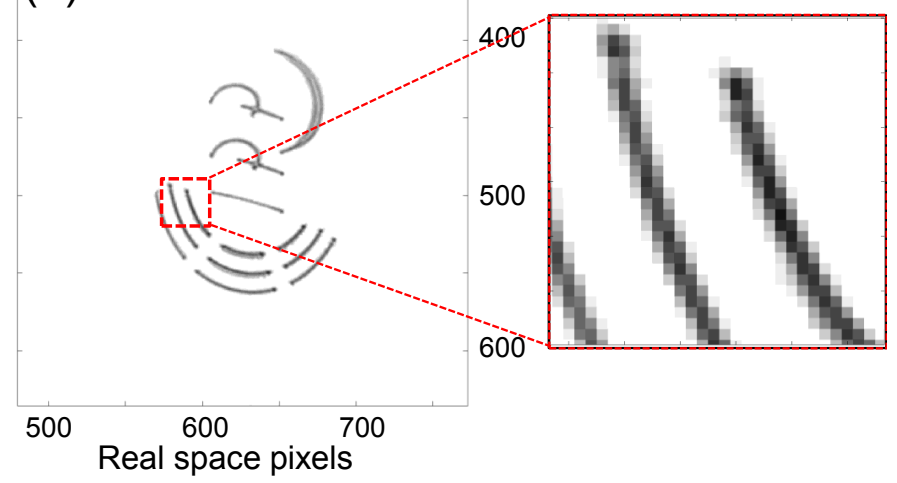

Figure 5.2: Coherent diffractive imaging using different illuminating wavelengths of $30 \mathrm{~nm}(\mathrm{a}, \mathrm{b})$ and $47 \mathrm{~nm}(\mathrm{c}, \mathrm{d})$.

size $d$ determine the fundamental limits for the resolution in lensless imaging, where $d$ is defined geometrically as $d=z \lambda / p N$. For a given pixel size $p$ of the CCD camera and the number of pixels $N$, the real space pixel size of the reconstructed image can be reduced if a shorter wavelength and/or a shorter distance between the sample and the camera are realized. Figure 5.2 compares two diffraction patterns of object A, recorded 
at a distance of $22 \mathrm{~mm}$ using the 27th [Fig. 5.2(a)] and the 17th harmonic [Fig. 5.2(c)] as a probe. The central parts of the corresponding reconstructions are shown in Fig. 5.2(b) and Fig. 5.2(c), respectively. While both reconstructions (as well as diffraction patterns) have the size of $1024 \times 1024$ pixels (after the curvature correction), the object size in the reconstruction, shown in Fig. 5.2(d), is smaller compared to that of the reconstruction shown in Fig. 5.2(b), corresponding to an increase of the real space pixel size from $30 \mathrm{~nm}$ to $\sim 50 \mathrm{~nm}$ and a reduced number of the effective pixels that constitute the object in real space. The difference in imaging quality of both reconstructions is apparent from the magnified parts of the reconstructions shown in the insets. On the reconstruction of the diffraction data recorded with shorter wavelength [Fig. 5.2(a,b)], finer features can be resolved. However this improvement is attributed not only to the reduced real space pixel size, but also to the improved spatial resolution, due to the increased momentum transfer that is available with shorter wavelength at the same $N A .^{2}$

A similar effect is observed if the distance $z$ from the sample to the detector is varied. This distance must be large enough to (i) ensure a sufficient oversampling and (ii) fulfill the requirements for the Fraunhofer diffraction approximation, meaning that the Fresnel number, $F$, has to be much smaller than unity, where $F=D^{2} / z \lambda$. Typically, the distance required by the Fresnel number is smaller than the one required by the oversampling conditions. The top row in Fig. 5.3 shows three diffraction patterns of object A, recorded at different distances: $12 \mathrm{~cm}, 8 \mathrm{~cm}$ and $6 \mathrm{~cm}$, whereas the bottom row features the magnified central areas of the corresponding reconstructions. Decreasing the distance $z$ linearly increases $N A$ and reduces the pixel size of the reconstructed image at the same time. In the actual experiment, this distance is chosen as a tradeoff between the desirable NA (to provide high resolution) and the oversampling ratio (ensuring the convergence of the phase retrieval algorithm). For the experimental conditions used, diffraction images with an oversampling ratio down to 4 can still be reliably reconstructed. For diffraction data with relatively low signal-to-noise ratio or noisy data, the distance must be larger to increase the oversampling ratio to 5 or greater, since this procedure improves the convergence of the reconstruction algorithm due to additional information redundancy. The reconstructions of highly oversampled data appear less noisy. These observations are in agreement with previously published studies investigating the influence of the oversampling ratio on

\footnotetext{
${ }^{2}$ Note that, regardless of the imaging resolution, the intensity distribution at the exit surface of the object of the given geometry is strongly wavelength dependent, due to waveguiding effects arising within the depth of the structure. Further details are discussed in Section 5.2.
} 
$12 \mathrm{~cm}$
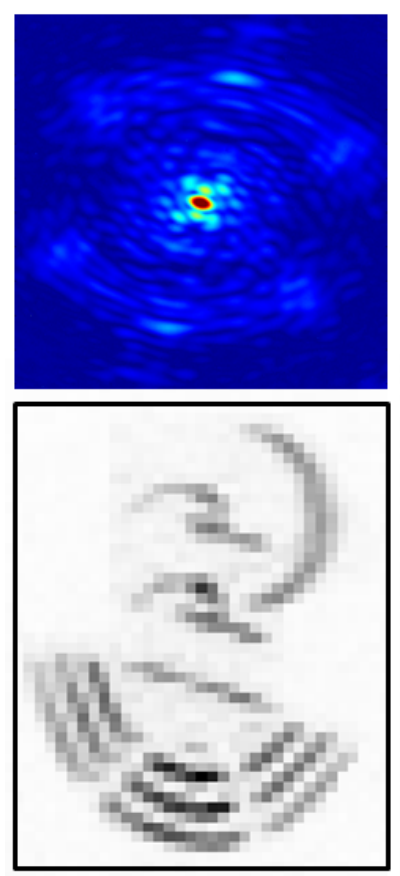

$8 \mathrm{~cm}$
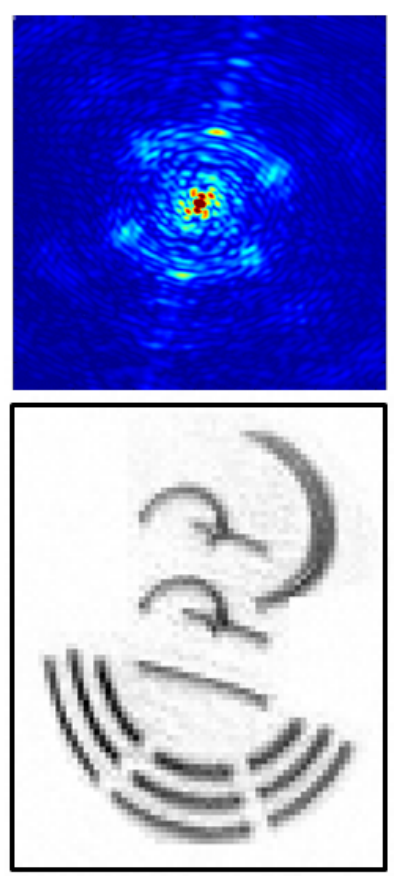

$6 \mathrm{~cm}$
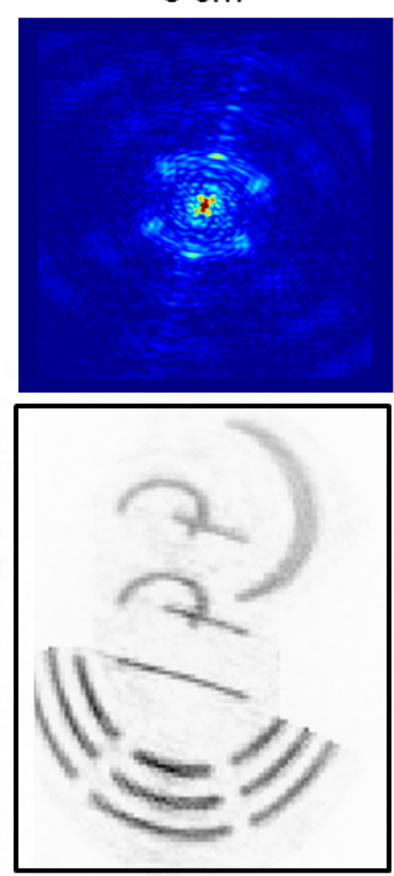

Figure 5.3: Dependence of the spatial resolution on the distance between the sample and detector. Top row: Diffraction patterns recorded at 12,8 and $6 \mathrm{~cm}$ distance using the same illuminating wavelength of $32 \mathrm{~nm}$. Bottom row: corresponding reconstructions.

the reconstruction [59].

Meticulous adjustments of the reconstruction parameters can force convergence to a "satisfactory" reconstruction result, especially when easily stagnating algorithms, e.g., Error Reduction or its modifications, are used in combination with a tight real space support. However, a subsequent run with the same parameters with a different starting point (first guess or a real space support) does not necessarily converge to a similar solution or converge at all. Generally, a higher signal-to-noise ratio of the diffraction pattern yields better the convergence of the phase retrieval algorithm. A low signal-to-noise ratio at high spatial frequencies diminishes the ability of the phase retrieval algorithm to effectively use the entire scattered signal. Therefore, in such cases, only the region of low spatial frequencies can be reliably reconstructed. This means that the achieved resolution does not depend on the geometric numerical aperture (the distance between the sample and detector, and its size), but on the $N A$ that is effectively used by the phase retrieval algorithm.

To estimate the highest spatial frequency that is still consistently reconstructed, the 

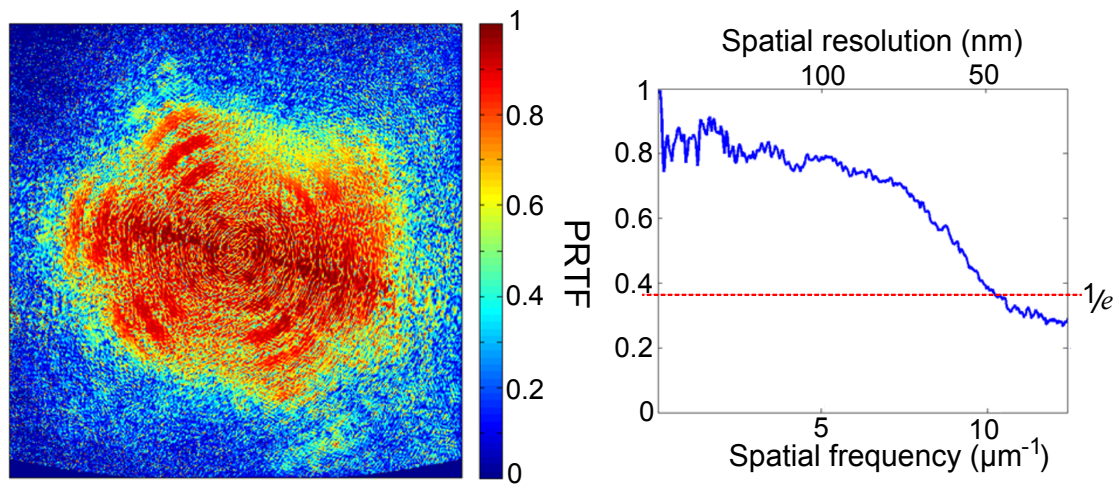

Figure 5.4: Resolution estimate with the phase retrieval transfer function (PRTF) showing the highest spatial frequencies that are consistently reconstructed.

phase retrieval transfer function (PRTF) can be used (see Chapter 3). Figure 5.4 shows typical values of the two-dimensional PRTF function for 100 individual reconstructions using the same parameters and a random first guess. Here, values above $1 / e$ imply a consistent reconstruction. In this reconstruction run, the effective $N A$ efficiently used by the iterative algorithm is lower than that, provided by the geometry of the detection scheme. The radial average plotted as a function of the spatial frequency can serve as a rough estimate for the accuracy of retrieved phases. In this case, spatial frequencies only up to $10.5 \mu \mathrm{m}^{-1}$ can be considered as reliably reconstructed, which corresponds to a half pitch resolution of $48 \mathrm{~nm}$. Tuning the reconstruction parameters for experimental data with rather low signal-to-noise ratio can only scarcely improve the reliability of the phase retrieval algorithm, demanding a further improvement of the detection scheme.

To increase the dynamic range of the diffraction pattern for weakly scattering objects, e.g., biological samples, and to reach a high signal-to-noise ratio even at high spatial frequencies, beam blocks can be implemented. In addition to that, several acquisitions can be accumulated and averaged. The first method, however, precludes the utilization of low frequency components for an estimation of the autocorrelation support. The second method proved to be useful for the measurements presented in Chapter 3. Figures 3.6(a) and 3.6(c) compare a diffraction pattern obtained by a single acquisition and an average over 200 individual acquisitions with 5 seconds exposure time each, i.e., a high dynamic range (HDR) image. The insets highlight an improvement of the signal-to-noise ratio at high spatial frequencies. This increases the effective $N A$ used in the phase retrieval algorithm and, consequently, improves the reconstruction quality, despite the fact that the highest 
momentum transfer that can be resolved on both images is identical. Figures 3.6(b) and 3.6(d) show reconstructions of the corresponding diffraction data displayed in Fig. 3.6(a) and 3.6(c). Both reconstructions are in good agreement with SEM images of the sample. However, Fig.3.6(c) reveals fine sub features that are not resolvable in the low-resolution reconstruction depicted in Fig. 3.6(b).

Apart from the resolution limitation, the convergence of the phase retrieval algorithm is improved for the HDR diffraction pattern and is not overly sensitive to the reconstruction parameters. This feature becomes much more pronounced for diffraction data exhibiting an intense small-angle scattering component. For example, as shown in the inset of Fig. 3.4(e) object B has a relatively large open area, which forms the very intense central part in the far-field. For the single acquisition diffraction pattern, the signal-to-noise ratio of the high spatial frequency components is very low, even when the central part of the diffraction pattern is close to the saturation limit $\left(2^{16}\right.$ counts for the employed 16-bit CCD camera). When such a diffraction pattern is used for reconstruction, data points with low or absent signal do not contribute to the reconstruction procedure. This can be treated as a reduced number of useful equations from the finite set that is used by a phase retrieval algorithm to reconstruct the given number of variables. In this case, the convergence probability is much lower and additional information is required. This information can be obtained from a better defined support, supplementary constraints and, of course, an increased oversampling ${ }^{3}$. In fact, provided a sufficient sampling, the missing information can be retrieved even for areas with no signal, e.g., a missing central part obscured by a beam block or additional pixels appearing after the curvature correction is applied. For example, the diffraction pattern used for the reconstruction shown in Fig. 3.2(b) has more than $5 \%$ pixels with missing information (pixels added to the corners due to curvature correction - about $1 \%$ ) or zero intensity, as a result of the subtraction of the readout noise. This feature can be used to improve the resolution by reconstructing the original diffraction data with zero-padding beyond the detector limits. However, in this case, additional severe constraints must be used: an assumption of the real-valued object (valid only for hard X-rays), thresholding of the real space signal, and a well defined support. A successful reconstruction with substantially decreased real pixel size (by a factor of 4) was recently reported [165].

\footnotetext{
${ }^{3}$ In the case of ptychography, a substantial overlapping of the diffraction patterns from different illuminating positions provides sufficient redundancy required for reconstruction with very high consistency of the retrieved data.
} 
For the measurements shown in Fig. 5.2(a,c), the achieved resolution is $\sim 30 \mathrm{~nm}$. The improvement over the resolution demonstrated in imaging experiments described in Chapter 3 is associated with the decreased illumination wavelength from 34.8 to $29.6 \mathrm{~nm}$. Although the distance between the sample and detector was different for these two measurements, the actual numerical aperture used for the reconstruction was rather similar. However, the reconstruction shown in Chapter 3 exhibits less noise due to slightly higher oversampling. Using the available photon flux at shorter wavelengths and benefiting from high temporal coherence available with the source developed, CDI of smaller structures with significantly improved spatial resolution is already feasible.

\subsection{Lensless imaging of the guided modes in the EUV spectral range}

The overall shapes of the reconstructed images shown in Fig. 3.2 are in good agreement with scanning electron micrographs of the used samples. However, a close inspection of the retrieved phases and amplitudes reveals a strong modulation, which is only resolvable on the reconstructions from HDR diffraction data. In Chapter 3, it is demonstrated that these modulations are associated with the mode beating of the EUV light transmitted through the nanoscale structures in the form of propagating modes. To corroborate our findings, the individual features of the object were modeled as nanoscale slits to perform numerical simulations of the light propagation in the vicinity of optically thick media. Additionally, a semi-analytical solution of the Maxwell equations for light propagation in two-dimensional slab waveguides is provided. Here, the propagation constants $\beta_{T E}$ and $\beta_{T M}$ are computed for transverse electric (TE) and magnetic (TM) modes separately, with the simulations incorporating the optical properties of the materials used in the experiments. Further details on waveguiding phenomenon can be found in Chapter 3 and in the literature, e.g., in Ref. [148].

Figure 3.4 shows plots of the propagation constants for gold-cladded vacuum-core slab waveguides of different widths. For a given wavelength, the width and length of the waveguide define the total contribution of each individual mode to the exit surface wave. The superposition of the propagating modes at the exit surface forms the field distribution emerging from the sample that will appear on the reconstruction. For higher order modes (and narrower slits) the effective propagation angles of the guided modes are larger [157]. 
The multiple scattering together with angle dependent reflection coefficients of the waveguide walls lead to a strong suppression of higher order modes in narrow waveguides. Thus, high aspect ratio waveguides effectively act as mode filtering devices. This feature can already be observed in the far-field images shown in Fig. 5.2(a) and Fig. 5.2(b), where the cutoff of the Fourier components occurs earlier for a longer illumination wavelength.

In Fig. 3.3(c) three specific features of the sample A, marked by lines L1, L2 and L3 in Figs. 3.3(a,b) are investigated in detail. Electric field profiles of the reconstructed exit wave are compared with the numerically simulated electric field distributions at the exit surface of the waveguides with dimensions and optical properties corresponding to the experimental conditions. Depending on the waveguide width, a different number of propagating modes contributes to the total electric field at the exit surface. Thus, mode beating at the exit surface of the sample is imprinted in the reconstructions as well. From the results presented in Chapter 3, it is apparent that CDI can be used to directly image propagating modes and near-field effects at the EUV spectral range with high precision. This feature can be advantageous for the investigation the electric field distributions in, e.g., photolithographic masks for the micro- and nanoelectronic industry.

Waveguiding and the associated mode filtering effect are essential for extruded objects and may influence the achieved resolution in CDI experiments. In the case of very narrow slits, where only a fundamental mode is supported (e.g., L1 in Fig. 3.3), the reconstructed feature will have a transverse profile of the fundamental mode, i.e., a Gaussian profile, although the actual profile of the slit is a step function. In this case, a strong damping of the higher order modes defines the sharpness of the finest feature of the reconstructed image. The so-called knife-edge technique, commonly used to estimate the achieved resolution, is therefore not applicable for real structures with a finite thickness. As apparent from Fig. 3.3, the actual profile on the reconstruction will depend on the thickness of the object and the associated Fresnel number.

\subsection{Polarization contrast in nanoscale structures}

The simulations of propagation constants shown in Fig. 3.4 predict a difference in transmission between TE and TM modes. In contrast to metal wire-grid polarizers for visible and infrared light [157], where TE modes are reflected by the polarizer surface due to high electric conductivity along the metal wires and TM modes are transmitted, the frequency of the EUV light is considerably higher than the plasma frequency of metals. Thus, both 
modes with the electric field pointing parallel (TE) as well as perpendicular (TM) to the slit are efficiently coupled into the slit (waveguide). The polarization contrast arises during the wave propagation and exponentially increases with the propagation through the waveguide. This contrast can be treated as a consequence of the difference between Fresnel reflection coefficients for TE and TM modes at the waveguide cladding. Figure 5.5(a) shows the simulated Fresnel reflection coefficients of gold surface at $40 \mathrm{~nm}$ wavelength. The reflection at small incidence angles is weaker, which is consistent with the stronger damping of the higher order modes that have (i) larger propagation angles inside the waveguide and, therefore, (ii) subjected to more reflection events compared to the fundamental mode within waveguide lengths. The reflection of S-polarized light, corresponding to the TE mode in the waveguide is much higher than the reflection of the P-polarized light (TM mode), which causes the polarization contrast of the waveguide after few reflections from the waveguide walls. The effective propagation angle of the fundamental mode also increases with a stronger optical confinement in narrower waveguides, reducing the total transmission through the waveguide and, at the same time, increasing the contrast between the transmitted intensity of TE and TM modes for the given wavelength and optical properties of the cladding material. Furthermore, the ratio between the reflected intensity of $\mathrm{S}$ and P-polarized light, is strongly dependent on the refractive index of the material used.
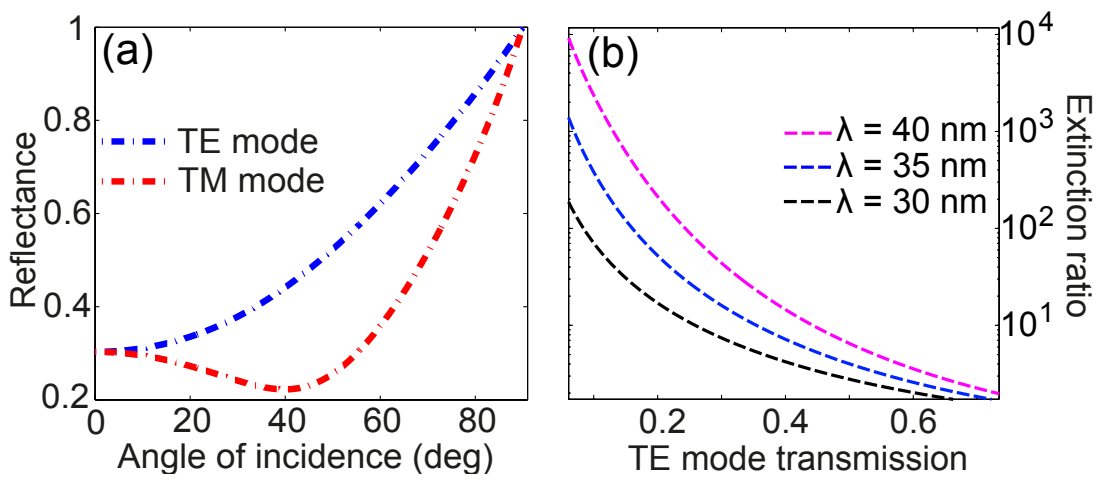

Figure 5.5: Polarization contrast in nanoscale slab waveguides. (a) Computed Fresnel reflection coefficients for $40 \mathrm{~nm}$ wavelength incident on different angles onto the gold surface. (b) Polarization contrast of nanoscale $300 \mathrm{~nm}$-long waveguides of different widths plotted as a function of the TE mode transmission for illuminating wavelength $\lambda$.

Higher losses for TM modes are associated with a longer penetration depth of the electric field to the optically dense cladding material where the electric field decays exponentially. 
A stronger penetration of the TM mode to the waveguide walls follows the continuity of the tangential electric field component perpendicular to the boundary (cladding surface). For the structures investigated in Ch. 4 , the intensity of higher order modes at the exit surface of the waveguides is negligible due to the strong damping, thereby only the fundamental modes contribute to the waveguide transmission.

Using experimental data and analytical simulations discussed in Ch. 4 we establish that polarization by selective absorption arising in nanoscale structures is sufficient for effective polarization filtering of EUV radiation. Figure 4.4(d) shows the computed extinction ratios, i.e., the ratio between the transmitted intensity of TE and TM modes, as a function of illuminating wavelength. Solid circles indicate the values obtained experimentally whereas the solid lines are the computed values for the structure corresponding to the experimental conditions ( $200 \mathrm{~nm}$ of Silicon Nitride with a $140 \mathrm{~nm}$-thick gold film). Interestingly, the values of extinction ratios measured experimentally fit to the curves corresponding to the computations performed for 38 and $52 \mathrm{~nm}$-wide waveguides. Such accuracy is beyond the resolution limit of the scanning electron microscope used to image the waveguide based structures, but agrees well with the SEM measurements showing 40 and $50 \mathrm{~nm}$ widths, respectivelly. Note that for the structure with $40 \mathrm{~nm}$-wide waveguides the extinction ratio is measured experimentally from the total signal, scattered from all the slits. For the illuminating wavelength of $38 \mathrm{~nm}$ an extinction ratio of $(28 \pm 1)$ is experimentally demonstrated. Using only gold as a cladding material will increase the achieved polarization contrast while minimizing the losses of the TE mode. Figure 5.5(b) shows the simulated extinction ratio as a function of the TE mode transmission for $300 \mathrm{~nm}$-long hollow-core slab waveguides with a gold cladding. Here, the extinction ratio of more than 1000 is already available with reasonable $15 \%$ transmission at $40 \mathrm{~nm}$ wavelength and further increases with wavelengths, highlighting the potential of such waveguides for polarization filtering applications in the EUV spectral range.

Clearly, utilization of nanoscale waveguides for polarization filtering may encounter some experimental challenges. Although the state-of-the-art nanolithography and chemical etching techniques enable production of large-area structures with closely arranged high aspect ratio slits, forming transmission gratings [166, 159, 167], the total geometrical transmission of any structure containing nanoscale waveguides is reduced due to a required separation between the waveguides and, e.g., extra material to ensure sufficient rigidity of the structure. Also, the transmitted light scatters at very high angles and is strongly modulated by the periodic grating structure. This might further reduce the avail- 
able polarized photon flux for applications that require a collimated beam, since only the central part of the scattered light will be used.

Another interesting effect inherent to waveguiding phenomena is a phase velocity difference between TE and TM modes. By tuning the parameters of a waveguide, such as cladding materials and geometrical dimensions, a specific waveguide-based device can be designed to provide a relative phase shift between TE and TM modes by, e.g., $\pi / 2$. This intrinsic phase shift converts the polarization of light incident onto the waveguide from linear to circular. Utilization of such an effect is particularly attractive in the EUV spectral range where the lack of strongly birefringent materials and high absorption of all materials impedes the development of EUV waveplates [168, 169]. However, the main challenge associated with the utilization of EUV waveplates based on nanoscale waveguides is the polarization sensitive absorption as discussed above. Specifically, the polarization state of the EUV radiation scattered from a waveguide is at most elliptical due to generally high ratios between the transmission of TE and TM modes.

Utilizing the polarization anisotropy originating in metallic nanosctructires, a waveguide-based linear polarization analyzer consisting of narrow slits oriented at different angles is developed. Due to a strong two-dimensional confinement and a sufficient azimuthal displacement of the slits, fields scattered from the differently oriented slits in the form of diffraction streaks are easily distinguishable in the diffraction pattern, especially at high spatial frequencies (cf. Fig. 4.2). Each diffraction streak direction is formed by the light scattered from slits perpendicular to the streaking direction. Thus, when illuminated with polarized light, the relative difference between the intensities of light streaking at different angles gives the information on the angle between the light polarization and slit orientation. Compared to conventional reflection-based EUV polarizers [27, 152], such an analyzer has several advantages: First, it is a very compact device with high misalignment tolerance; Second, it provides information on the polarization of the incident light in a single acquisition. With the growing interest in the recently developed generation schemes for elliptically and circularly polarized harmonics [29, 28, 30, 34, 35], the proposed analyzer is an advantageous tool for fast polarization measurements, enabling a reliable in situ optimization of the generation conditions. Furthermore, in combination with a reflection-based waveplate, e.g., the gold surface of the diffraction grating in the HHG source presented here, this analyzer can distinguish between light with circular and random polarization.

In order to demonstrate the direct relation between the electric field distribution at the 

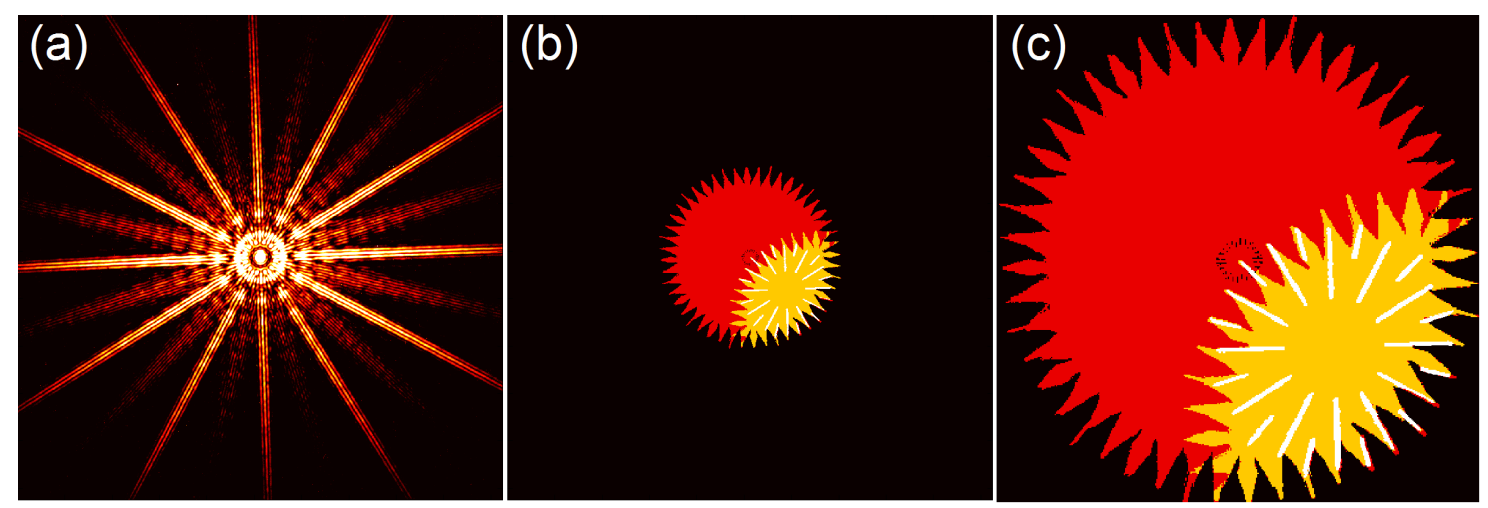

Figure 5.6: Deconvolution of the autocorrelation support. (a) Measured far-field intensity. $(b, c)$ Autocorrelation (red), deconvolved autocorrelation support (orange) and an envelope of the reconstructed structure (white).

exit surface of the developed analyzer and an intensity distribution in the far-field, coherent diffractive imaging was implemented. Using the autocorrelation support, we reconstruct the exit surface wave of the analyzer from the far-field diffraction formed with $35 \mathrm{~nm}$ wavelength light. Figure 5.6 illustrates the steps required for the reconstruction. First, a low-pass filter is applied to the inverse Fourier transform of the measured intensities shown in Fig. 5.6(a) to find the autocorrelation of the object [shown in red in Fig. 5.6(b,c)] which is twice the size of the object in each dimension. Second, the real space autocorrelation support [depicted in orange color in Fig. 5.6(b,c)] is deconvolved from the autocorrelation of the sample. Figure 5.6(c) represents a zoomed inner part of the Fig. 5.6(b). The thresholded reconstruction is depicted in white color in Fig. 5.6(b,c) for illustration purposes, but its envelope can be further used as an updated and much better defined real space support to speed up and improve the convergence of the phase retrieval algorithm. Figure 4.2(c) shows the intensity distribution at the exit surface of the analyzer. The polarization sensitive slit transmission estimated from the real space reconstruction is in very good agreement with the data obtained from the measured far-field [Fig. 4.4(c)]. To our knowledge, this is the first demonstration of polarization sensitive CDI using high harmonics. Measurements shown in Fig. 4.3, where the incident light polarization was changed while the sample remained fixed, rule out possible influences of the scalar transmission difference between the slits or a strong tilt of the sample. Due to the high aspect ratio of the slits, a deviation of the angle of incidence from normal incidence by $9^{\circ}$ already excludes the geometrical transmission of some slits. However, due to the effective coupling of the fundamental mode, illumination even at higher angles does 
not diminish the waveguide transmission. Our numerical simulations show that a sample misalignment by $9^{\circ}$ to the incident beam, reduces the transmission of the fundamental modes only by $10 \%$.

\subsection{Capabilities and limitations of the developed source for lensless imaging}

The generated spectrum of the source developed can be easily extended to shorter wavelengths using noble gases with a higher ionization potential (cf. Eq. 1.4), e.g., neon or helium. Figure 5.7 shows a spectrum generated in neon. Due to the higher ionization potential $I_{p}$ compared to argon $(21.6 \mathrm{eV}$ vs $17.8 \mathrm{eV})$ the cutoff energy $E_{\text {cutoff }}$ of HHG from $\mathrm{Ne}$ is at much higher photon energy and reaches values beyond the absorption edge of the aluminum filter at $17 \mathrm{~nm}$. Due to a higher ionization potential, a higher field intensity is required to initiate the HHG process. The available photon flux is comparable to that generated in argon, which is sufficient for CDI experiments with just few seconds exposure time. For wavelengths below $17 \mathrm{~nm}$, replacement of the aluminum filter with, e.g., zirconium filter will improve the available photon flux by a factor of 60 at $10 \mathrm{~nm}$ wavelength due to a much higher transmission in the wavelength range between 7 and $17 \mathrm{~nm}$. However, in this case, a rather low thermal conductivity of zirconium (10 times lower than that of aluminum) increases the damage probability of the filter due to overheating. This issue can be solved, for example, using a $\mathrm{Zr}$ filter coated with few nanometers of silver that has high reflection coefficient at $800 \mathrm{~nm}$ as well as a very high thermal conductivity. According to Eqs. 1.1 and 1.2 the spatial resolution of an imaging system scales linearly with the illuminating wavelength $\lambda$ for a given numerical aperture. However, the linear oversampling required for phase retrieval algorithms (cf. Eq. 1.3) also scales linearly with $\lambda$, and in order to compensate a reduction in oversampling ratio $O$, caused by a shorter wavelength, a larger distance $z$ between the sample and detector is needed. Let us estimate a possible improvement of the imaging resolution for sample A described in Chapter 3, if the illuminating wavelength is decreased from $35 \mathrm{~nm}$ down to $17 \mathrm{~nm}$ corresponding to the lowest wavelength with high photon flux available without any modification of the experimental setup. Reconstruction of the diffraction data with $O<5$ already becomes challenging [59], which means that decreasing the illuminating wavelength by approximately a factor of two will require an increase of the distance $z$ also by a factor of 2 


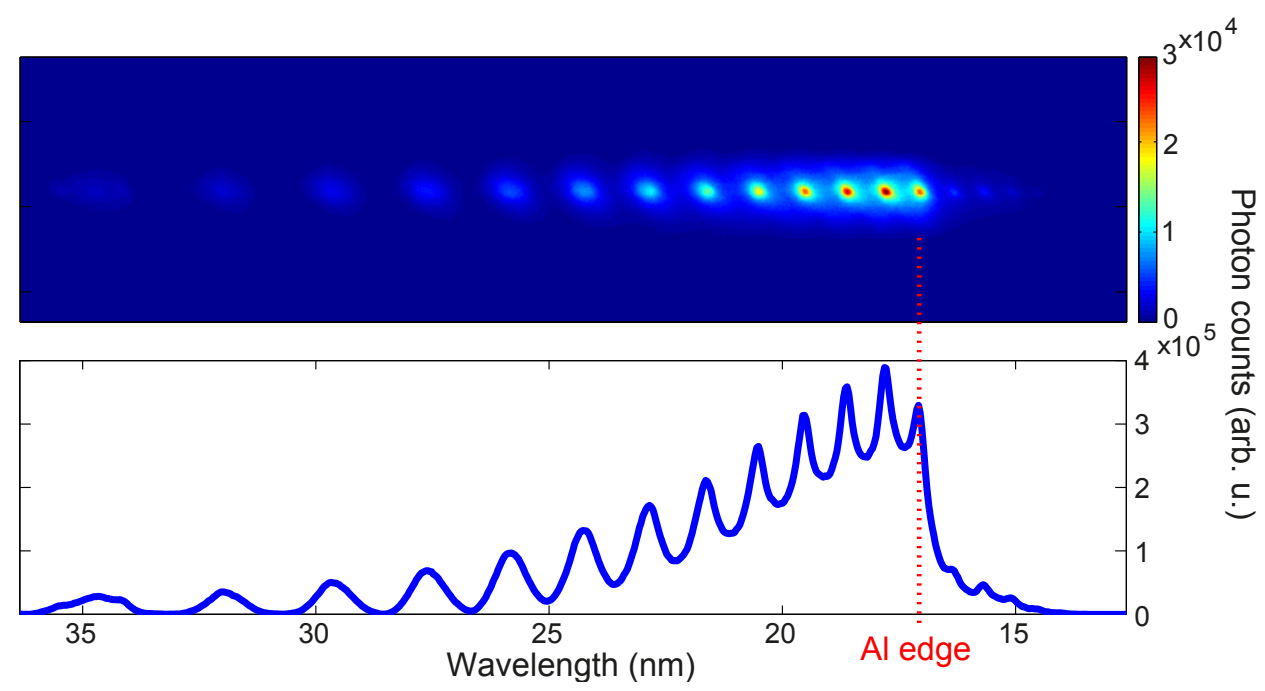

Figure 5.7: Spectrum of high harmonics generated in neon with $0.8 \mathrm{~mJ}$ pulses spanning beyond the aluminum absorption edge.

or more, in order to keep the oversampling ratio high enough ${ }^{4}$. For the given detector size, such an increase of sample-to-CCD distance will inevitably reduce the numerical aperture, which governs the achievable spatial resolution according to Eq. 1.1. Thus, for $8 \mu \mathrm{m}$ sample and fixed oversampling, decreasing the illuminating wavelength from $35 \mathrm{~nm}$ down to $17 \mathrm{~nm}$ leads to a resolution gain of only $5 \mathrm{~nm}$ from 35 to $\sim 30 \mathrm{~nm}$. Further reduction of the illuminating wavelength does not improve the spatial resolution available in CDI with the given detector parameters (cf. the blue line in Fig. 5.8, where the achievable spatial resolution is plotted as a function of the illuminating wavelength for the given geometrical parameters of the detector used).

On the other hand, for a smaller field of view (smaller object size), decreasing the illuminating wavelength will not be that crucial for oversampling of the diffraction pattern. For example, reduced illuminating wavelength from 35 to $17 \mathrm{~nm}$ will improve the resolving power available in CDI by a factor of two if the field of view is smaller than $4 \mu \mathrm{m}$, because the oversampling ratio is high enough even at the reduced wavelength. Furthermore, the distance between the sample and the detector can be also reduced while still ensuring a sufficient oversampling as well as the validity of the Fraunhofer approximation for the scattered signal. Thereby, CDI is favorable for rather small samples.

\footnotetext{
${ }^{4}$ In results shown in $\mathrm{Ch} .3$ the oversampling ratio, $O$, that ensures consistent and reliable convergence of a phase retrieval algorithm was $\sim 4.4$ corresponding to the distance $z=2 \mathrm{~cm}$ and the illuminating wavelength of $35 \mathrm{~nm}$ for $8 \mu \mathrm{m}$-long sample.
} 
(a)

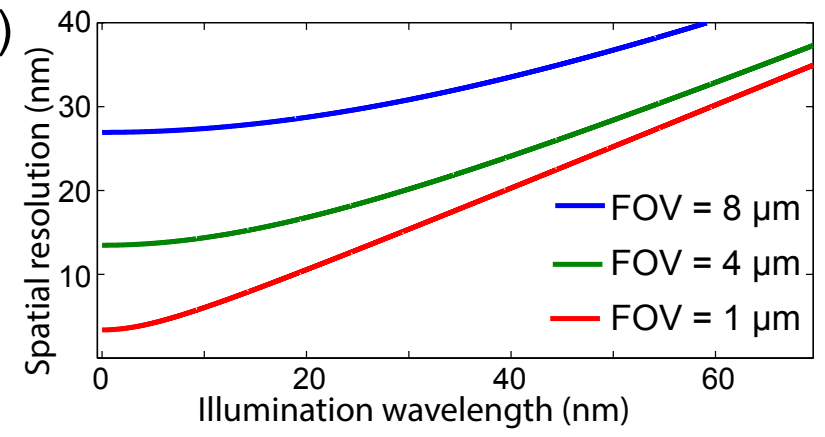

(b)

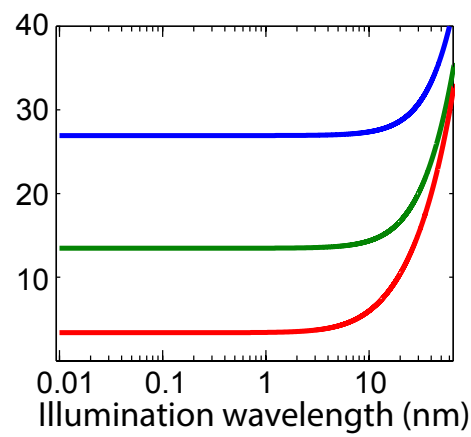

Figure 5.8: Capabilities of lensless imaging technique. (a) Spatial resolution theoretically available via CDI for given CCD parameters at the constant oversampling ratio $(O=4.4)$, plotted as a function of illumination wavelength for a field of view (FOV) of 1, 4 and $8 \mu \mathrm{m}$; (b) Same as (a) on a logarithmic scale.

Figure 5.8 illustrates the achievable spatial resolution via CDI as a function of the illumination wavelength (at the fixed oversampling ratio of 4.4 and used CCD parameters) for different sample sizes (fields of view) of 1,4 and $8 \mu \mathrm{m}$. Using the current experimental setup without any modifications, coherent diffractive imaging with sub $10 \mathrm{~nm}$ resolution is, in principle, already available for objects with lateral dimensions of $1.5 \mu \mathrm{m}$ or smaller using the 47 th harmonic order $(\lambda=17 \mathrm{~nm})$. In this case, however, very high scattering angles up to $60^{\circ}$ have to be recorded. Such an improvement of the spatial resolution is beyond the values demonstrated by any EUV table-top microscope. The best spatial resolution reported to date is $22 \mathrm{~nm}$ [117], achieved using $13 \mathrm{~nm}$ wavelength for $1 \mu \mathrm{m}$ test object and an advanced CCD detector with 13.5 micron pixel size to further improve the oversampling ratio. Recently shown results on imaging of $\mu \mathrm{m}$ scale structures indicate the possible improvements available via a larger detector size and a narrow field of view [170].

\subsection{Chapter summary}

To conclude, coherent diffractive imaging is a very powerful tool enabling the investigation of a transmission function of specimens, using a variety of contrast mechanisms with a resolution, in principle, limited only by diffraction. The local field distribution at the exit surface is reconstructed from the far-field diffraction pattern after the illumination of the object with highly coherent radiation. The utilization of the short illumination wavelength and detection of scattered light at high numerical aperture are key aspects 
for high-resolution reconstructions. The distance between the sample and the detector is chosen not only to satisfy the oversampling requirements, but also to provide a sufficient signal-to-noise ratio over the entire diffraction pattern. The high dynamic range diffraction data is reconstructed with a diffraction-limited resolution - down to the illuminating wavelength of $30 \mathrm{~nm}$. The high quality of reconstructed images is attributed to the enhanced monochromaticity of the illuminated beam due to the utilization of a diffraction grating instead of a multilayer mirror. This allows for a thorough investigation of the object's transmission function which shows that the reconstructed images are strongly affected by waveguiding phenomena arising within the object itself. Therefore, reconstructions differ from the geometry of the object expected from the scalar transmission. Furthermore, the sharpness of the reconstructed features are reduced by a damping of higher-order waveguide modes. To estimate the resolution of the obtained images, a phase retrieval transfer function is used. This method evaluates the effective numerical aperture that is consistently contributing to the reconstruction.

We demonstrate that high aspect ratio structures exhibit a strong polarization anisotropy in the EUV spectral range, which can be observed locally with high accuracy directly at the sample surface using CDI. This introduces a new degree of freedom in terms of contrast forming mechanism for lensless imaging using an HHG source, thus enabling, e.g., magnetic imaging using a table-top HHG source.

A polarization contrast of metallic nanoscale slab waveguides in the EUV spectral range is exploited in a design of a new type of linear analyzer for extreme ultraviolet radiation. Such a device provides an information on the polarization of high harmonics by means of a single acquisition measurement enabling in situ optimization of the generation conditions. 


\subsection{Future prospects}

\section{EUV photon flux optimization}

The presented results on diffractive imaging, obtained with only 5 seconds exposure time (corresponding to 5000 laser shots) reveal the potential of the developed source for a single-shot non-repetitive imaging. In order to reduce the exposure time, while still enabling high signal-to-noise ratios, a significant optimization of the EUV photon flux density at the sample is required. Part of the performed studies on the source optimization and characterization were conducted in close collaboration with T. Mey (Laser Laboratorium Göttingen), with some of the results presented in his work [171], as well as in Chapter 2. In the following, the possible strategies for improving the photon flux and discuss primary modifications to the source, which have been carried out after the experiments described in Chapters $2-4$.

The most common way to characterize light sources is to estimate the brilliance, which is often referred to as the source's quality. The Brilliance $\boldsymbol{B}$ accounts for the photon flux of a given energy bandwidth per unit area and divergence angle and is given by:

$$
\boldsymbol{B}=\frac{\text { Photons }}{\operatorname{second} \cdot \mathrm{mrad}^{2} \cdot \mathrm{mm}^{2} \cdot 0.1 \% \mathrm{BW}}
$$

where $B W$ is the bandwidth of the central frequency of the emitted radiation and mrad is the divergence of the beam. By analogy with large scale light sources, the brilliance is a convenient measure for the quality of the HHG source as well. Although in special cases an influence on the divergence angle and the spectral bandwidth of high-harmonic radiation was observed [105, 172], in general, divergence and bandwidth are mainly governed by the optical elements used in the generation scheme, specifically the numerical aperture of the focusing lens, and the beam transport of the high harmonics (diffraction grating or multilayer mirrors). Thus, only the photon flux of the generated spectrum is relevant for HHG. Moreover, the central parameters of high harmonics, including the spatial coherence, the mode profile, and the degree of polarization, are proven to be crucial for lensless imaging as well.

The spectral bandwidth, provided by the diffraction grating, is directly related to the total number of slits illuminated. To improve $\lambda / \Delta \lambda$ at the sample position, the generation point of high harmonics was located further away from the diffraction grating in order to increase the harmonic spot size on the grating surface. At the same time this decreases the 
distance, $x$, between the grating and its focal spot downstream, increasing the numerical aperture of the grating. A simple equation for a thin lens can be used to estimate the achieved gain: $1 / a+1 / x=1 / f$, where $a$ is the distance between the generation point and the rating, and $f$ is the focal length of the toroidal grating which is $16 \mathrm{~cm}$ for the grating used. Since the beam waist is linearly dependent on the numerical aperture, increasing the distance $a$ decreases the spot size of the harmonic beam at the focus of the grating, therefore improving the photon flux density at the focal spot. Furthermore, increased distance $a$ helps to overcome experimental challenges associated with utilization of highly fragile free-standing aluminum filter placed between the generation point and the grating. Any misalignment of the optical elements used add aberrations to the high-harmonic beam and therefore reduce the available photon flux density at the focal spot. An illustrative example is shown in Fig. 2.3 where the available irradiate is plotted as a function of the grating misalignment.

High photon flux is essential not for all applications of the source due to the direct relation of the photon flux to the achievable signal-to-noise ratio of the recorded data. For a given repetition rate of the driving laser, the strategy for improving the high-harmonic flux is obvious - namely to increase the efficiency of the frequency conversion as well as the generation volume and to minimize the potential losses. The proper control of the propagation conditions of the fundamental laser beam including laser intensity, the interaction length and pressure covers all three aspects and corresponds to the macroscopic phase-matching optimization described in Chapter 1. However, a single atom response which drives the HHG process is not related to phase-matching conditions and will be discussed in more details.

Wavelength scaling of HHG efficiency. Several numerical studies have predicted a strong scaling of the single-atom high-harmonic yield $Y$ with the pump wavelength as: $Y \propto \lambda^{-5.5 \pm 0.5}$, mainly governed by the quantum dispersion of the electron's wave packet $[173,174]$. Depending on which parameters are kept unchanged (laser intensity, ponderomotive energy, etc.), a variety of HHG scaling laws and deeper insights into the topic of wavelength scaling HHG yield can be found in theoretical [175, 176, 177, 178, 179, 180] as well as experimental works [173, 107, 181, 182, 183]. Furthermore, Frolov et al. have pointed out that a number of severe misconceptions in the theoretical quantification arise from the definition of the harmonic yield itself $[184,179]$. However, despite such systematic study of the wavelength dependence of the HHG yield came out in recent years, 
an experimental quantitative confirmation remains challenging. First of all, it is difficult to design an experiment where the driving wavelength is allowed to be the only changing parameter while HHG depends non-linearly on a number of parameters. Second, it is very challenging to differentiate in the experimental data the contributions between the single atom response and macroscopic phase-matching. From a practical point of view, in terms of using a high-harmonic source for applications, we are interested in the potential gain of photon flux of a certain harmonic for the given power of the pump laser.

To study the wavelength dependence of HHG, we performed measurements, in which a barium borate (BBO) crystal was used to partially convert the initial $800 \mathrm{~nm}$ laser light to $400 \mathrm{~nm}$ wavelength via a second harmonic generation process. In the experiment, the isolated second harmonic of the fundamental beam was used alone or combined with the residual $800 \mathrm{~nm}$ beam to drive the HHG process. The BBO crystal was chosen due to its properties, including a high transparency for 800 and $400 \mathrm{~nm}$ wavelengths, a large nonlinear coefficient, a broad phase-matching range, and a high damage threshold. The thickness of the nonlinear crystal plays a crucial role in the frequency doubling and must be chosen accordingly to the experimental requirement, i.e., to ensure maximum intensity of the second harmonic pulse for subsequent HHG. This is provided by the optimization of conversion efficiency of the second harmonic generation ( $\mathrm{SHG}$ ) while maintaining the initial pulse duration.

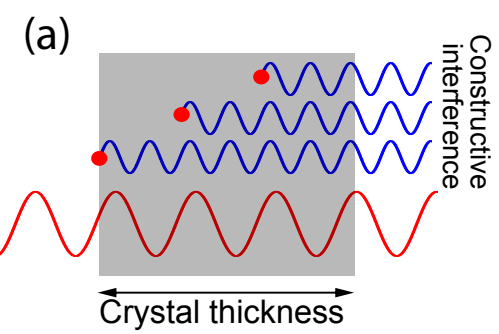

(b)

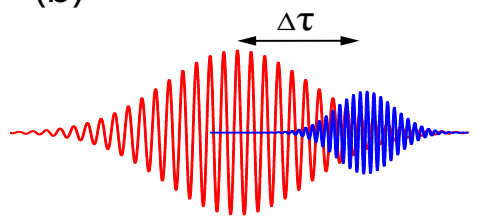

Figure 5.9: (a) Schematic illustration of phase matching for second harmonic generation. (b) Illustration of a temporal walk-off $\Delta \tau$ of ultrashort pulses resulted from the phase velocity mismatch.

The dependence of the SHG efficiency on the crystal thickness can be understood from Fig. 5.9(a), which illustrates a simplified scheme of the efficient frequency doubling in the case of a plane wave illumination. When the fundamental beam with frequency $\omega$ (shown with red color) propagates through the nonlinear crystal, the signal of the second harmonic (with frequency $2 \omega$ ) increases, due to constructive interference of the signal 
generated along the crystal medium at phase-matched conditions. In this case, SHG is more efficient for thicker crystals. However, in the case of the ultrashort pump pulses used in our experiments, the group velocity mismatch between the primary pulse and its second harmonic imposes severe restrictions to the useful interaction length (crystal thickness). Since two pulses with different central frequencies $\omega$ and $2 \omega$ propagate through the BBO crystal at different velocities, the so-called temporal walk-off, i.e., the temporal delay between the pulses will monotonously increase. Figure 5.9(b) illustrates the temporal walk-off between the two pulses. Clearly, the overlap between the pulses will fade out after some propagation distance, inevitably influencing the conversion efficiency of the SHG process.

An additional constraint to the crystal thickness in the case of ultrashort pulses is associated with chromatic dispersion and the finite bandwidth of the femtosecond pulses, which is related to the pulse duration via a Fourier transformation. Since the phase matching can be fulfilled only for a very narrow frequency range of the entire spectrum of the fundamental pulse, the nonlinear medium acts as a bandpass filter, reducing the bandwidth of the generated pulse and, therefore, increasing the pulse duration of the second harmonic.

Fortunately, the high intensity of femtosecond laser pulses drastically increases the nonlinear response of the nonlinear crystal, which mitigates the losses associated with the reduced crystal thickness. Our computations and experimental data show that a $200 \mu \mathrm{m}$ thick BBO crystal is a good tradeoff for the used amplified laser system, and we obtain a high conversion efficiency of up to $40 \%$ using the collimated pump beam. The high damage threshold of the BBO crystal allows for an further gain of the conversion efficiency, which can be obtained by reducing the pump beam diameter on the BBO crystal. However, in this case, additional optical elements may introduce aberrations to the laser beam and influence the subsequent HHG process.

When the second harmonic of the fundamental laser light is used do drive the HHG process, we observe a dramatic increase of the harmonic yield in the plateau region. For these measurements, only the driving wavelength was changed, but this inevitably changes the focusing parameters, thus influencing the intensity in the focus as well as the interaction length. The following phase matching optimization (tuning the capillary pressure and incident pump power intensity) is easier for short wavelengths $\lambda$ of the driving field. This is attributed to the reduced plasma dispersion, which is proportional to $\lambda^{2}$. This also means that slightly higher laser intensities can be used before the ionization level reaches the 
critical value [107], further improving the generated EUV flux.

As expected from the three step model described in Chapter 1, when the short wavelength pump is used to drive the HHG process, the generated spectrum is much narrower. For instance, for the given gas, the maximum photon energy, $E_{\text {cutoff }}$, available through the HHG process from a single atom is determined by the ponderomotive energy $U_{p}$. This energy scales with the driver intensity $I$ and wavelength $\lambda$ as $E_{\text {cutoff }} \propto I \lambda^{2}$. Thus, the utilization of a high frequency driver lowers the maximum photon energy of the HHG source. Fortunately, an increase of the laser intensity, possible due to a higher critical ionization level, can partially mitigate the effect of the reduced driving wavelength on the ponderomotive energy and slightly extend the cutoff frequency. Eventually, the "real" cutoff energy, governed by the macroscopic phase-matching, is not as favorable to the driver wavelength as it is determined by the single atom HHG scaling: $E_{\text {cutoff }} \propto \lambda^{2}$. A previously reported approximate power law $\lambda^{1.6-1.7}$ can serve as a reference [185].

To summarize, the microscopic single atom response in the HHG process is significantly increased when short wavelength radiation is used as a driver, leading to a favorable change of the generated EUV photon flux. Therefore, a shorter wavelength, for example, the second harmonic of the fundamental $800 \mathrm{~nm}$ beam, is recommended to drive the HHG process, if the corresponding cutoff frequency is high enough to reach the desired photon energy.

Polychromatic driver. Another possible route to increase the conversion efficiency of the HHG process and to even extend the cutoff frequency is the optimization of the driver's waveform. Experimentally, this can be accomplished when two or more optical fields of different frequencies are coherently superimposed. In the tunneling ionization regime, this effect has been first observed in 1994 [186] with a combination of the fundamental beam with frequency $\omega$ with its third harmonic $(3 \omega)$. The resulting total flux was increased by one order of magnitude, depending on the intensity ratio and the relative phase between the two light fields. Recently, it was shown that - compared to the single monochromatic driver - such a wavelength combination has the potential to enhance the HHG flux by two orders of magnitude over the entire spectrum and by one order of magnitude when the fundamental beam is combined with its second harmonic $[187,188,189]$. Furthermore, analytical studies have proposed an "Ideal Waveform" that can be "designed" using five octaves of the fundamental laser frequency. Without increasing the total laser power, such an optimization due to intercycle shaping of the laser waveform 
will generate recollision energies that are 3 times higher than those from a sinusoidal single frequency beam [190].

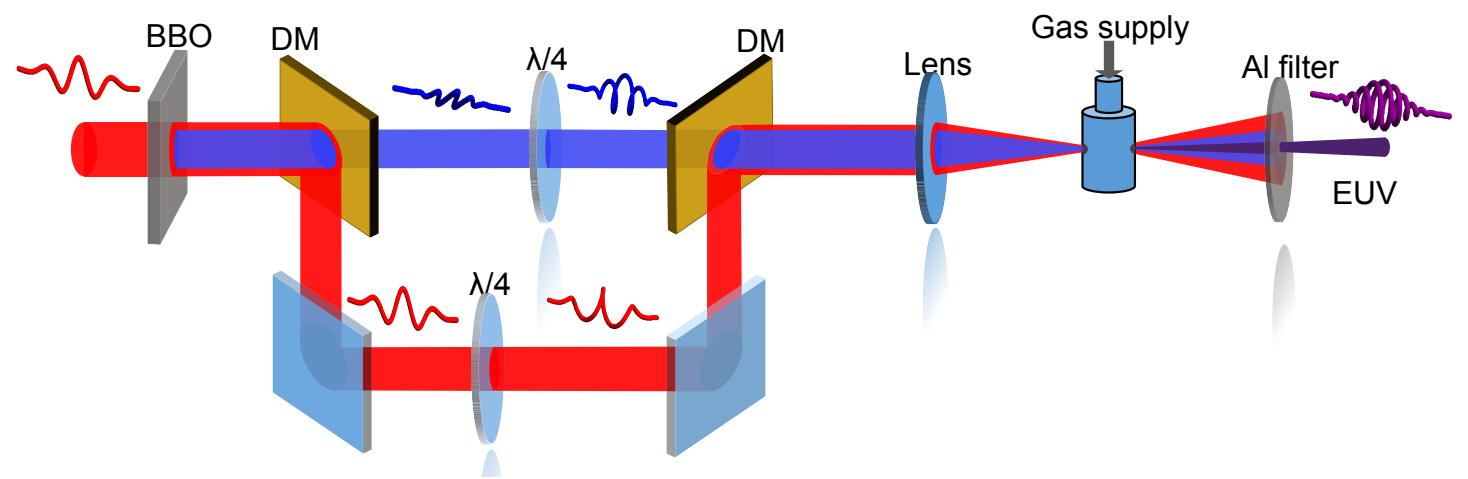

Figure 5.10: A generation scheme for circularly polarized harmonics. The second harmonic of the fundamental $800 \mathrm{~nm}$ laser beam is generated in the BBO crystal and separated using a dichroic mirror (DM). Both beams are converted to circularly polarized radiation using quarter-wave plates $(\lambda / 4)$ and later combined using the second DM to generate circularly polarized high harmonics. The temporal overlap between the pulses is provided by an adjustable delay of the fundamental beam.

As described in Chapter 1, the fundamental beam of a given frequency $\omega$ generates only odd harmonics due to inversion symmetry. Additional fields, for example, from bichromatic drivers as discussed above and/or a presence of weak static fields [191], break the symmetry, allowing for generation of the even harmonics. Most importantly, such a bichromatic generation scheme helps to overcome the limitation of conventional HHG to linear, or at most slightly elliptical, beams [192] opening up a new route for tuning the polarization state of the generated harmonics. Since the efficiency of the HHG process strongly depends on the probability for a tunneled electron to recollide with its parent ion (see Ch. 1), it is not possible to generate high harmonics with monochromatic circularly polarized light, as in this case, the recollision probability is completely suppressed. The situation changes when two circularly polarized beams (rotating in opposite directions), with frequencies $\omega$ and $2 \omega$ are used to drive HHG. The resulting superposition of the light fields drastically increases the probability of a tunneled electron to recollide with its parent ion, compared to the elliptically polarized field with a single frequency. Due to spin angular momentum conservation [27], the generated harmonics exhibit a strongly elliptical polarization, and may, in principle, reach an ellipticity of 1 , provided a circular polarization of both drivers at the generation point. As recently demonstrated, such generation schemes have already proven useful for polarization sensitive spectroscopy, 
including circular dichroism $[193,30,35]$ and are very promising for magnetic imaging using lensless techniques.

In our experiments, where a linearly polarized fundamental $800 \mathrm{~nm}$ wavelength beam with a frequency $\omega$ was combined with its second harmonic $(2 \omega)$ to drive the HHG process, a noticeable enhancement of the signal of generated harmonics is observed as well as the change in the generated spectrum, i.e., the appearance of even harmonics predicted by the theory when the temporal overlap between the pulses is minimized. Figure 5.11 displays the spectra recorded under different generation conditions using a $800 \mathrm{~nm}$ beam together with its second harmonic (with and without temporal overlap). When the pulses are not temporary overlapped, the detected spectrum corresponds to the sum of the spectra generated separately with $\omega$ and $2 \omega$, since the temporal delay between the pulses prevents the interference of their respective electric fields. However, the intensity of the given harmonic order is lower when two beams are used. This can be explained by the fact that the delayed pulse interacts with the already pre-ionized gas medium, which causes a strong defocusing. This effect is more pronounced when the $\omega$ beam is delayed, because the critical ionization level is lower for $\omega$ compared to $2 \omega$. When two fields of $\omega$ and $2 \omega$ are temporally overlapped, the HHG flux is significantly increased together with the emergence of even harmonics [cf. Fig. 5.11(b)]. The increase in the efficiency can be also associated with additional nonlinear processes that are now available and can contribute to HHG. Our observations are in good agreement with theory as well as previously reported data [33].

When the driving laser fields are converted to circular polarization with opposite helicity, the generated spectrum reveals a suppression of every third harmonic, indicating the selection rule for circularly polarized $\operatorname{HHG}[33,27]$. However, the total photon flux of the non-suppressed harmonics is reduced by more than two orders of magnitude compared to signal of HHG from linearly polarized light of the same intensity. This observation does not meet the recently reported scaling [34], where the circularly polarized harmonics flux is lower than that of the linearly polarized by a factor of three. We attribute these unexpectedly high losses to the unfavorable intensity ratio of the fundamental field with respect to the field of the second harmonic. Although the power ratio between the two beams was in good agreement with Ref. [34], the position of the focal points in the generation volume were spatially displaced by more than $8 \mathrm{~mm}$ due to chromatic aberration of the employed focusing lens. The difference between the generation schemes, i.e., the gas cell in our case and hollow core waveguide in Ref. [34], can be neglected. 


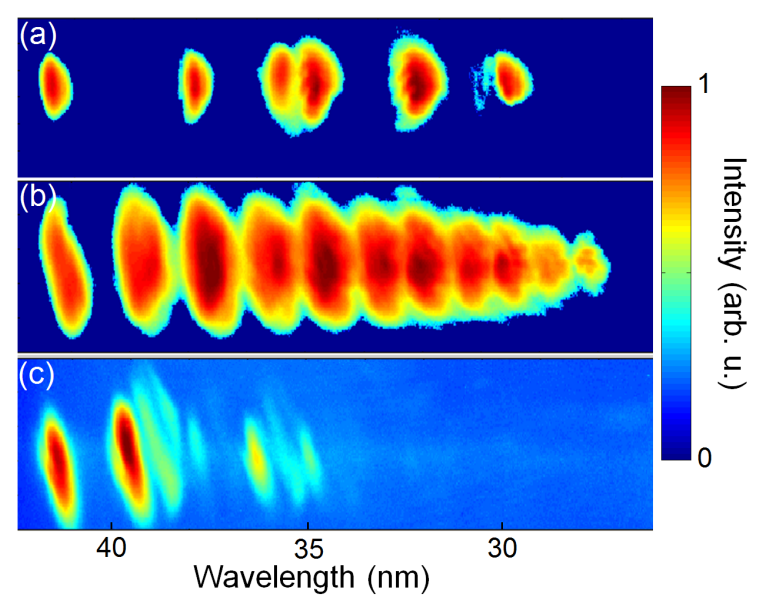

Figure 5.11: (a) Spectrum of high harmonics generated in argon when two laser beams with frequencies $\omega$ and $2 \omega$ are collinearly polarized, but not temporally overlapped. (b) Same as (a), but with temporal overlap of the pulses. (c) Spectrum recorded with circularly polarized drivers, exposure time is increased by two orders of magnitude.

To overcome this limitation, a special achromatic lens is required. Such a lens should be designed to maintaining the circular polarization after passing the lens. Furthermore, the group delay dispersion of the thick achromatic lens can be a limiting factor, because the pre-chirp of the laser pulses is tuned to optimize the pulse duration at the BBO crystal, which means that any dispersive element downstream will broaden the laser pulses at the generation point. Alternatively, a parabolic mirror as well as a separate focusing of the individual beams before the last dichroic mirror can be used [33]. The latter will allow for a precise control of the field intensity ratios by tuning the focal positions of both beams relative to each other. Our measurements show that a pre-adjustment of the beam divergence of one of the beams with Galilean telescope introduce additional optical aberrations that drastically reduce the beam quality of the generated harmonics.

To conclude, the utilization of a bichromatic driver for HHG strongly improves the overall conversion efficiency and allows for the generation of frequencies forbidden in conventional monochromatic HHG schemes. However, this generation scheme imposes additional complexity to the optical beam path and alignment, which drastically influences the stability and the beam quality of the emitted harmonics. This can also explain the fact that it took almost 20 years after its first experimental demonstration [33] to implement such a scheme in applications [193, 30, 35]. Nevertheless, the bichromatic generation design currently presents the most promising way to control the polarization state of the 


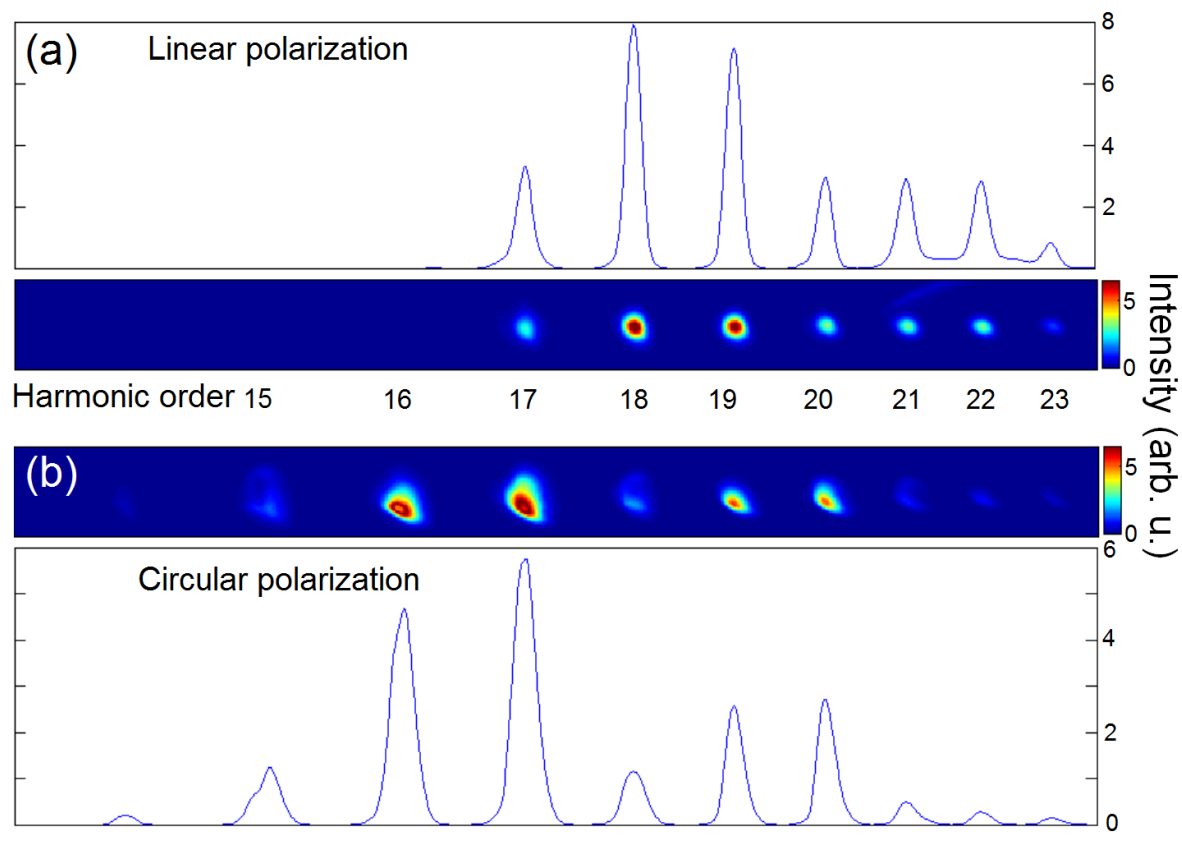

Figure 5.12: (a) Spectra of high harmonics recorded in Ar when driving laser beams with frequencies $\omega$ and $2 \omega$ are collinearly polarized. (b) Spectrum recorded with circularly polarized drivers. The suppression of every third harmonic indicates the selection rule for the circularly polarized HHG.

generated harmonics and, compared to the use of reflection based waveplates [168, 194], is not limited to narrow bandwidths. This ultimately enables the generation of elliptically polarized light with variable ellipticity in the entire harmonic spectrum.

\section{Towards table-top magnetic imaging}

Using the recently proposed scheme, a generation of circularly polarized harmonics is available with a much simpler scheme that does not involve a Mach-Zehnder interferometerlike geometry and which is tolerant to small misalignment [195]. As an emergent result of collaborative efforts with the group of Prof. Oren Cohen (Technion - Israel Institute of Technology) during the productive visits of Ofer Kfir, such an innovative inline generation scheme was implemented within our experimental setup. This enabled us to increase the conversion efficiency of HHG for circularly polarized harmonics - a crucial step in the path towards spatial and, perhaps, spatio-temporal investigation of chiral phenomena and as magnetism. In the implemented apparatus, a BBO crystal is placed after the focusing lens, which eliminates the chromatic aberrations that appeared to be the limiting factor in 
the aforementioned measurements involving the bi-chromatic driver. The polarization of both beams of fundamental $800 \mathrm{~nm}$ light and its second harmonic, is converted from linear to circular via a dual-band quarter-wave plate. The control of the temporal overlap is available through the adjustment of two group-velocity-delay-compensation plates made of calcite.

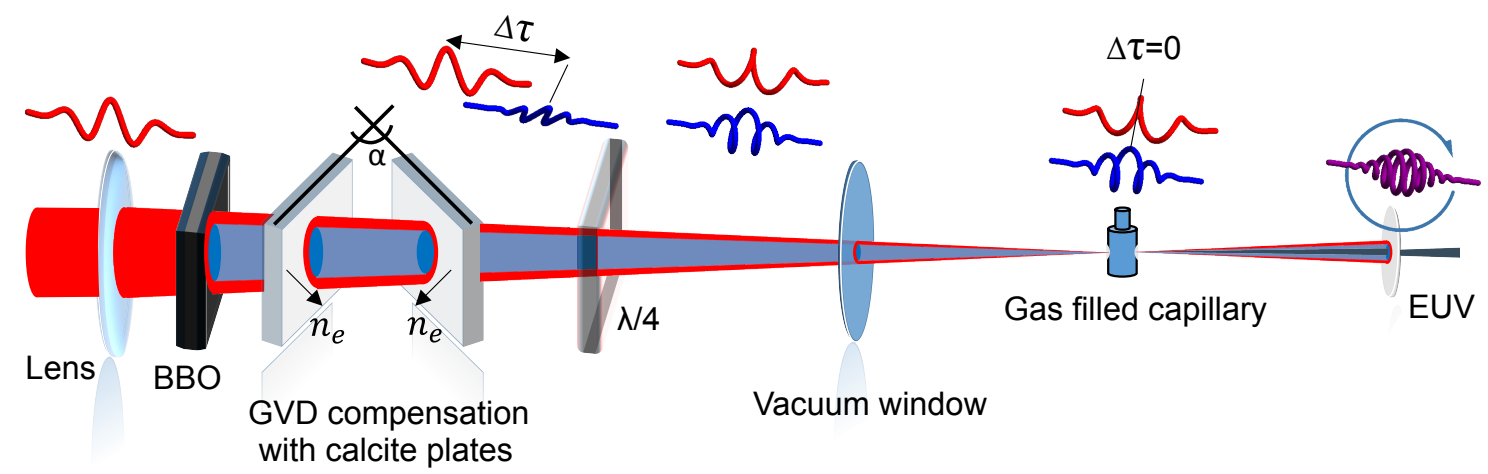

Figure 5.13: Compact in-line generation scheme for circularly polarized harmonics. The pulses of the second harmonic of the fundamental laser light generated in the BBO crystal are temporally overlapped with the fundamental pulses using a delay-compensation plates with an adjustable angle $\alpha$. The polarization state of both frequencies are converted to circular using an achromatic quarter-wave plate $(\lambda / 4)$. Adapted from Ref. [195].

Using such a device and an increased energy of the laser pulses up to $3 \mathrm{~mJ}$, we observed HHG emission with a high photon flux, spanning up to the 46th harmonic order corresponding to $71 \mathrm{eV}$ (limited by the transmission the aluminum filter) and covering M-edges of several transition metals such as iron, cobalt and nickel [196]. Figure 5.14(a) shows two HHG spectra obtained in Ar (green solid line), $\mathrm{Ne}$ (red dashed line) and He (blue solid line). The observed suppression of every 3rd harmonic indicates the selection rule for circularly polarized HHG. The measured photon flux of the 38th harmonic (photon energy of $\sim 60 \mathrm{eV}, 3 p$ M-edge of Co) exceeds $10^{6}$ photons/pulse or $10^{9}$ photons/second at the detector, with a full-width-at-half-maximum of the harmonic beam at the focal spot of the diffraction grating smaller than $\sim 30 \mu \mathrm{m}$. The available photon flux in such a broad spectrum, together with easily controlled helicity of the generated harmonics, paves the way towards imaging of magnetic structures with nanoscale spatial resolution using a compact source of EUV radiation. One of possible routes techniques for investigation of the magnetic samples with nanometer-scale spatial resolution is Fourier transform holography (FTH). After its first implementation to magnetic imaging by Stefan Eisebitt and co-workers in 2004 [71], FTH became a standard tool for high resolution imaging of the 

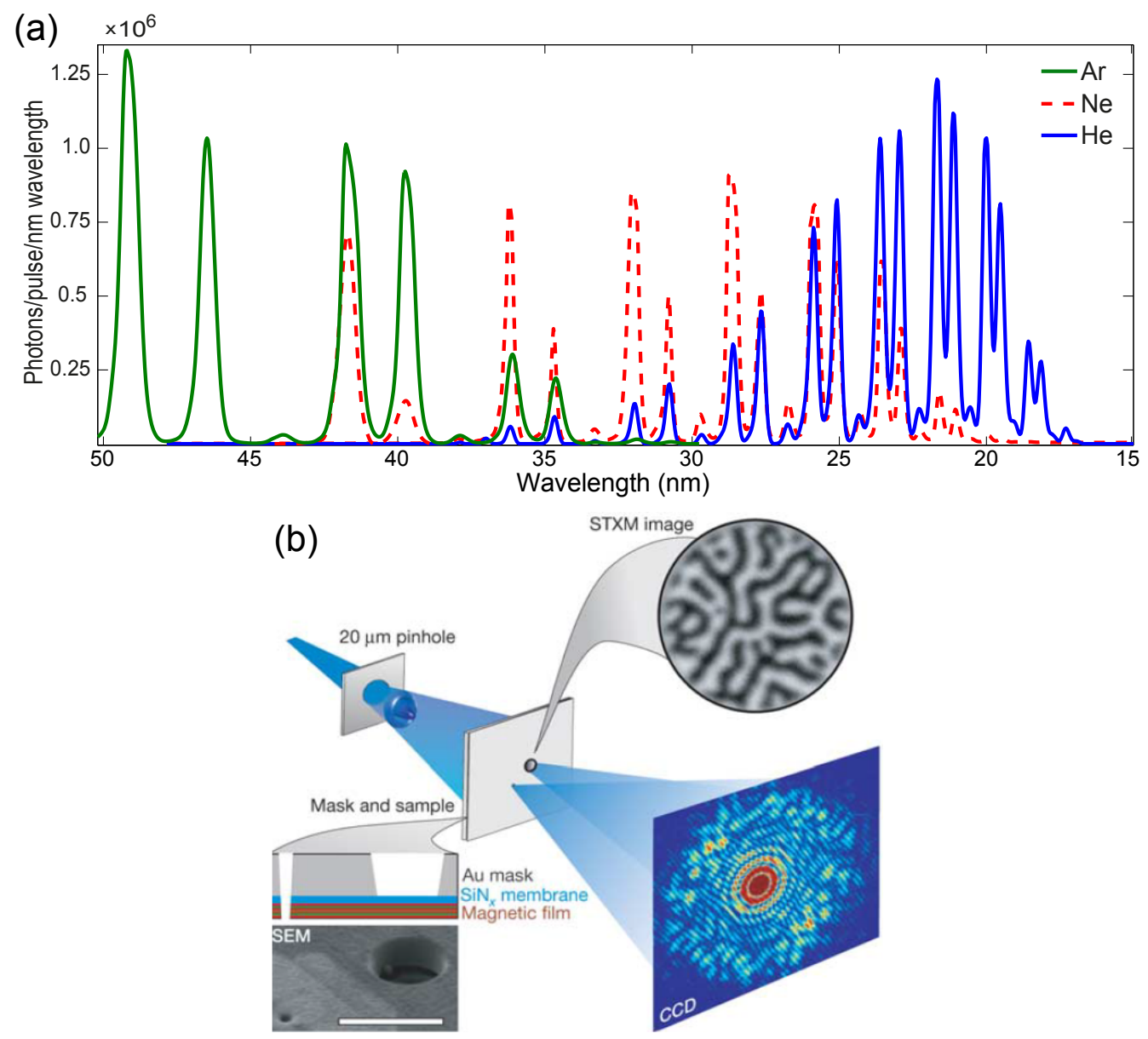

Figure 5.14: (a) Spectrum of circularly polarized harmonics generated in Ar (green solid line), $\mathrm{Ne}$ (red dashed line) and He (blue line) using a bi-chromatic inline generation scheme. (b) Schematic representation of the lensless imaging of magnetic domains is reprinted by permission from Macmillan Publishers Ltd: Nature (Ref. [71]), copyright (2004).

magnetic structures at the L-edges of $3 d$ transition metals [71, 197, 198] [cf. Fig. 5.14(b)]. Later, it was shown that FTH works equally well for the investigation of magnetic samples at the M-edge [199]. However, so far, magnetic imaging with high spatial resolution was demonstrated only at large scale facilities such as FELs and Synchrotrons. 


\section{Appendix A}

\section{Appendix}

\section{A.1 Experimental setup and preparations for operation}

The experimental setup in this work is based on three main components: the driving laser, the high-harmonic generation (HHG) chamber combined with a monochromator and an imaging chamber. In the following all components will be described separately, highlighting important aspects and required optimization procedures for imaging experiments.

\section{Laser system}

As a source for fundamental radiation we employ the commercially available regenerative amplifier "Legend Elite Duo", seeded with the Ti:Sapphire oscillator "Mantis", both manufactured by Coherent. The oscillator system generates $40 \mathrm{fs}$ long optical pulses with $800 \mathrm{~nm}$ central wavelength at $80 \mathrm{MHz}$ repetition rate using the passive Kerr-lens modelocking technique. Laser pulses with an approximate energy of $5 \mathrm{~nJ}$ are delivered to the built-in stretcher of the amplifier system (Legend Elite Duo). The spectral bandwidth of the oscillator can be optimized to match the cut of the spectrum, introduced by the stretcher. The optimization is performed by tuning the thickness of the positive dispersion element (glass wedges) inside the oscillator cavity. Reducing the thickness of the glass, which the intracavity soliton has to pass, narrows the generated spectral bandwidth and at the same time improves the ability of the oscillator to turn into a mode-locking regime at reduced pumping power. This also extends the cleaning intervals of the optical elements and improves the overall stability of the operating system.

The diffraction grating of the stretcher of the amplifier system is arranged with other optical elements in a way to direct the high frequency part of the spectrum over a longer path 
compared to the low frequency components, resulting in a positive chirping of the initial femtosecond pulse. The added chirp significantly reduces the peak power of the pulses to avoid optical damage to laser components downstream. Every 80,000th stretched pulse from the generated pulse train enters the amplifier cavity containing two Pockels cells. This is achieved by periodically applied high voltage to the first Pockels cell. The initial polarization of the stretched pulse is changed from vertical $(\mathrm{S})$ to parallel $(\mathrm{P})$ due to a double passing through the first Pockels cell, keeping the P-polarized pulse trapped inside the cavity of the regenerative amplification stage where a Ti:Sapphire crystal is used as an active medium. After 12-15 round trips (controlled by the delay of the second Pockels cell), depending on the alignment of the amplifier cavity and the contamination of the optical components, the second Pockels cell changes the polarization back to S, thereby releasing the amplified pulse. The pulse energy after the regenerative amplification is increased by six orders of magnitude, reaching a value of typically $5 \mathrm{~mJ}$. The amplified pulses are directed to the second optically pumped Ti:Sapphire crystal to double the achieved pulse energy via single pass amplification. At this stage, a proper overlap between infrared and the pump beams ensures the optimal output beam profile, which is crucial for the nonlinear processes involved in HHG experiments. Finally, the amplified pulses are recompressed in time by compensating the chirp that was introduced by the stretcher and evolved during the amplification process. This is done via the compressor, involving a diffraction grating. The resulting laser pulses come close to their original duration, enabling an average power up to $8 \mathrm{~W}$ at $1 \mathrm{kHz}$ repetition rate. The expected pulse duration (40 fs) of the laser system is confirmed using spectral phase interferometry for direct electric-field reconstruction.

Depending on the distance and the thickness of the dispersive elements between the laser system and the experimental setup, the pulse duration directly at the place of interest (in our case the capillary of the HHG chamber) requires an optimization of the pre-chirp adjustment, i.e., the overcompensation of the chirp by the compressor. Because of a compact arrangement of the optical elements inside the compressor, a noticeable beam cut from one side of the laser output profile is commonly caused due to a clipping of the laser beam at the movable retroreflector mirrors ensemble. One of the possible solutions to this issue is an additional round trip (available via a delay adjustment of the second Pockels cell) in the regenerative amplification cavity or the adjustment of the pulse buildup time, specifically, the reduction of the delay between the seeded and unseeded build-up times, which is, in principle, counter-intuitive and contradictive to the regular alignment 
procedure. The latest can be realized by walking the seed beam inside the regenerative amplification cavity. This will affect the pulse build-up time due to a changed overlap between the intracavity and the seed beam.

\section{High-harmonic generation chamber and monochromator}

The output of the laser beam has a full width at half maximum diameter of $\sim 9 \mathrm{~mm}$. A radial truncation with a fixed aperture size improves the efficiency of the harmonic upconversion by optimizing the laser focusing conditions [200, 201]. A truncation of the beam to 4-6 mm ensures the highest yield of the harmonic emission for the generation geometry used. The remaining average power of the laser light after the iris lies between 250 and $500 \mathrm{~mW}$ for experiments shown in Chapters 2-4. Such a strong truncation may induce distortions and intensity modulations of the beam profile caused by the thermal heat of the aperture and, consequently, a local Stack effect. However, noticeable distortions become apparent when the incident laser power exceeds $4 \mathrm{~W}$. Placing the aperture right before the last optical element (a focusing lens) minimizes the edge diffraction effects in the focal plane.

After the radial truncation, the laser light is focused with a $20 \mathrm{~cm}$ focal length lens into an argon-filled metal capillary. The dimensions of the capillary, specifically, its diameter $(6-9 \mathrm{~mm})$ and length $(20 \mathrm{~mm})$, were chosen empirically to maximize the HHG flux for certain generation conditions [111]. The walls of the capillary are not thicker than $150 \mu \mathrm{m}$ and can be burned through by the focused laser light from both sides to create the input and output capillary apertures. On one hand, this technique allows to circumvent a complicated alignment procedure as in the case of the existing aperture. On the other hand the apertures produced in situ by a laser beam ablation are very small - down to the size of the beam waist. When a new capillary is installed, the laser drilling should be done by slowly increasing the laser intensity to avoid excessive burning of the capillary and therefore reduce the contamination of the generation chamber and to ensure symmetric shape of the created apertures - one of the crucial parameters for the output of the harmonic emission, influencing the mode quality and harmonic yield. Figure A.1 compares images of the used capillaries, magnified by a factor of 20 using an optical microscope. The noticeable difference in aperture shapes caused by the beam pointing deviations significantly influences the efficiency and the beam profile of the high-harmonics yield.

Apart from the simplified alignment procedure which is reduced to a positioning of the 

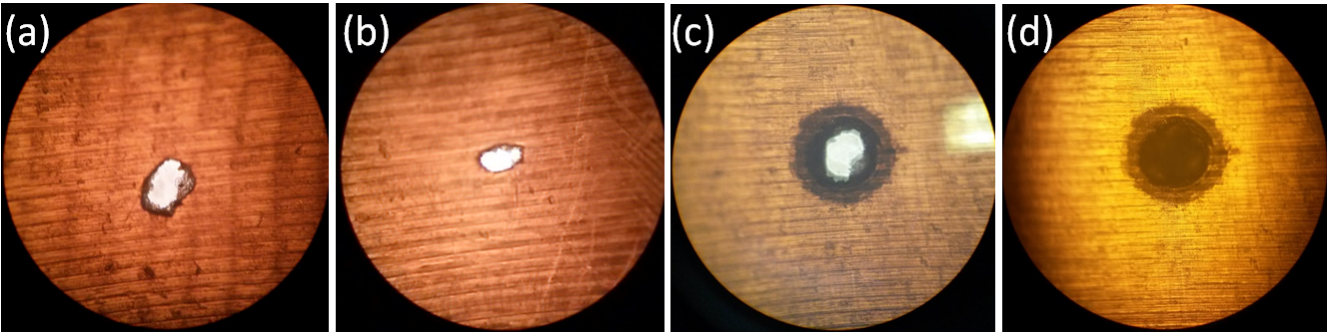

Figure A.1: Laser-induced ablation of the capillary walls. Images of the used capillaries taken with 20x magnification of the optical microscope. $(a, b)$ Exit openings, $(c, d)$ entrance apertures.

capillary perpendicular to the incident beam (provided by the detection of the back reflection of the alignment laser), the utilization of the capillary gives a number of advantages compared to alternative generation schemes for phase-matching optimization involving hollow-core waveguides or a gas jet:

1) The gas supply to the capillary is not limited to low pressures only and can be constant, releasing the necessity of complicated pulsed injection systems.

2) The pressure distribution along the propagation lengths is relatively homogeneous, allowing for proper phase matching conditions along the entire interaction length.

3) The diameter of the capillary can be optimized to match the interaction length and together with the sharp differential pumping, due to the small entrance apertures, significantly reduce the reabsorption of the generated harmonics. For example, at an argon pressure optimized for a proper phase matching at the used wavelengths of $35 \mathrm{~nm}$ to $\sim 150 \mathrm{mBar}$, the absorption length, i.e., the distance at which the intensity of the radiation drops to $1 / e$ of the original value, is less than $200 \mu \mathrm{m}$.

4) The intensity as well as the focusing conditions can be chosen arbitrarily, neglecting the possibility of the optical damage as in the case of the waveguide geometry.

However, there are a few disadvantages associated with the capillary scheme: first of all, the apertures of the capillary are continuously exposed to the very intense laser light and any beam pointing deviation or capillary vibrations will immediately burn the capillary walls. Therefore, the entrance window of the generation chamber and, more importantly, the aluminum filter downstream the capillary might be contaminated. However, in the latest experiments, this challenge was mitigated using a self-adhesive $20 \mu \mathrm{m}$ thick Copper foil to serve as the capillary walls. Second, the actual gas pressure inside the capillary can not be directly monitored, because for a given back pressure of the gas supply it depends on the aperture size of the capillary as well as on the number of apertures (in 
the case of the multiple usage of the same capillary). Despite the simplicity of the used generation scheme, i.e., the utilization of the pressure controlled phase matching inside the gas filled capillary, the obtained high harmonic flux and the beam quality, including the beam profile and spatial coherence, are comparable to the values achieved with the phase matching using hollow core waveguides [202, 203]. Surprisingly, a very similar HHG conversion efficiency and available photon flux was reported for phase matching optimization using a pulsed gas jet geometry [204].

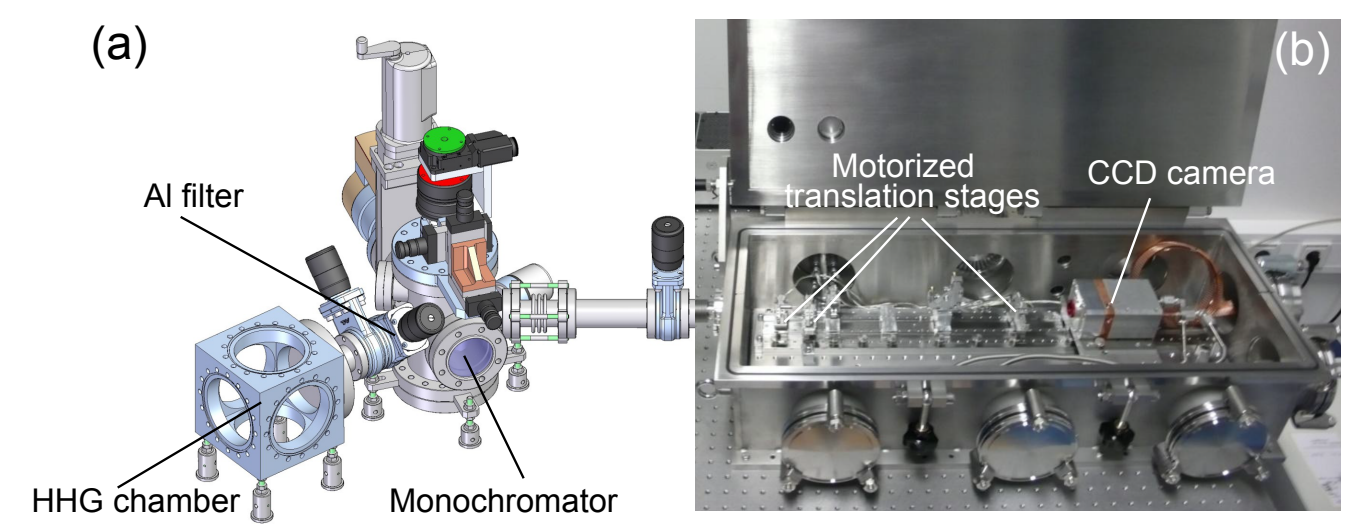

Figure A.2: (a) Experimental setup consisting of the generation chamber and a grating-based monochromator. (b) Picture of the imaging chamber.

Figure A.2(a) shows the experimental HHG setup. The generation chamber is evacuated with two sequential roughing pumps (not shown in the Figure) to a pressure of $10^{-3} \mathrm{mbar}$, whereas the monochromator is kept under high vacuum conditions $\left(10^{-7} \mathrm{mbar}\right)$ by a set of oil free membrane and a turbomolecular pump. High-harmonic radiation, generated in the capillary, propagates collinearly with the fundamental near-infrared laser light to the toroidal blazed diffraction grating. A free standing aluminum filter is introduced between the HHG chamber and the monochromator chamber to serve two purposes: to absorb the fundamental light and to separate the generation chamber from the high vacuum oil-free monochromator. The diffraction grating is mounted to a motorized rotation stage. By setting the angle of the diffraction grating, a desired harmonic order from the generated spectrum can be selected and isolated with the slit placed in the focusing point of the diffraction grating. The distance between the capillary, i.e., the generation point, and the diffraction grating is set to be two times the focal length of the toroid $(f=16 \mathrm{~cm})$, resulting in point to point imaging. The pulse broadening, caused by the grazing incidence angle of the toroidal grating can be compensated using the second identical diffraction 
grating set to the same incident angle, but at the opposite diffraction order. Note that for imaging experiments only the first diffraction grating was used to minimize the losses on the grating reflection. The estimated pulse broadening at $35 \mathrm{~nm}$ is $360 \mathrm{fs}$. Later, the generation point was placed further away from the monochromator to decrease the intensity of the fundamental laser beam on the Al filter and to improve the temporal resolution of the monochromator due to the illumination of larger number of slits of the diffraction grating. This also reduced the beam waist of the focused high-harmonic beam.

\section{Coherent diffractive imaging chamber}

The imaging chamber shown in Fig. A.2(b) is installed after the monochromator. Two motorized 2-D translation stages and one 3-D stage with a resolution step of $1 \mu \mathrm{m}$ can be used to position and manipulate a pinhole, the sample and a beam-stop if needed. To isolate one harmonic from the generated spectrum, a $1 \mathrm{~mm}$ wide slit is positioned close to the focus of the EUV light directly before the sample. The distance between the 23rd and adjacent harmonics is 1.97 and $1.64 \mathrm{~mm}$ at the focal spot.

The sample is mounded to a motor with 4 degrees of freedom - 3D translsation as well as a rotation axis. Prior to the imaging experiments, the harmonic flux can be optimized if the sample holder is rotated by 90 degree relative to the normal incidence, i.e., removing the sample out of the beam pass. This feature allows for optimization of the EUV flux prior to the experiments without the need of venting the chamber.

The CCD camera utilizes a back-illuminated EUV sensitive chip with an array of $1340 \times 1300$ pixels, $20 \mu \mathrm{m}$ each, operating at a 16 bit depth, thereby limiting the dynamic range of the single acquisition to 65536 counts. Thermoelectric cooling, available down to $-50^{\circ} \mathrm{C}$, substantially reduces the dark current resulting in a typical noise level of 105 counts per pixel per read out at $100 \mathrm{kHz}$ readout frequency. During the image acquisition with the CCD camera, the ion gauge of the CDI chamber and monochromator must be switched off as well as all piezoelectric motors to minimize the overall noise in recorded images.

\section{A.2 Optical alignment}

It is important to deliver high-harmonic radiation to the imaging chamber with minimum aberrations. Even a small misalignment in the optical system drastically reduces the qual- 
ity of the experimental results. The optical system is very sensitive to the lateral and angular alignment, for example, the rotation angle of the diffraction grating that is needed to change the output harmonic from 27th to 29th is below one tenth of a degree, same value of angle misalignment is capable to add astigmatism of more than $5 \mathrm{~mm}$ (c.f. Chapter 3$)$.

The alignment of the monochromator can be lost or degraded over time and therefore frequent adjustments are unavoidable. This section describes a step by step alignment procedure that can be used as an instruction for fine adjustments in future experiments to maintain the experimental facility in the operating condition without the external assistance or to reinstall the system in another location.

1. Two helium-neon lasers $(\mathrm{HeNe}$ ) have to be set horizontally (parallel to the optical table) to the same height, which will be the height of the entire system and positioned on both sides of the monochromator chamber so that the two beams cross at one point in the middle of the chamber, passing through the centers of the input and output flanges of the chamber. Note that the total angle between the flanges $\left(142^{\circ}\right)$ can be used as a reference. A metal tip with M6 thread was fabricated to fit to the chamber in order to identify the middle point. The level of the chamber can be measured at the top flange with the spirit level and adjusted if required.

2. The diffraction grating must be installed to the holder and positioned with 3-D manipulator in such a way that both laser spots are coincide in the middle and do not change the height of the reflected light. The normal incidence light must be reflected strictly to the opposite direction, coinciding with the incoming beam and the grazing incident light (when the grating is in the zeroth-order at $71^{\circ}$ incident angle) reflected to the middle of the second alignment laser. Two additional irises directly at the outputs of each of the HeNe lasers improve the alignment precision. Once the grating is positioned, the motor must be "set to zero" to save the current position. It is important that the zero position is at least three degree away from the mechanical reset point of the grating motor. Using the reflected light as the input beam, the second chamber of the monochromator with the diffraction grating can be positioned analogously, if needed. The distance between the centers of two gratins must be four times the focal length of the gratings which is $16 \mathrm{~cm}$.

3. When the alignment of the monochromator is completed, the vacuum chambers can be connected by a flexible tube with a VAT valve in the middle. A valve shutter supports an adjustable slit that will serve for isolation of a single harmonic order and should be placed in the middle between two gratings, i.e., in the focus, to a avoid possible clipping 
of the harmonic emission.

4. The generation camber must be placed in front of the first monochromator chamber to the correct height and leveled. The laser light should pass through the middle of the entrance window and the exit iris. Close the system of two grating chambers and a generation chamber with view-ports that are transparent for $800 \mathrm{~nm}$ wavelength light and evacuate the system. Due to the ambient pressure and a non-symmetric construction of the 3D manipulator of the grating holders, the alignment of the grating must be corrected using three additional adjustment screws. It is preferable to avoid the height correction with the 3D manipulator.

5. The lens that will be used in the experiment to focus the infrared light into the capillary should be placed in front of the generation chamber so that the focus is approximately in the middle of the chamber. Proper transverse alignment of the lens can be confirmed by the transmitted light, which should not change the direction of propagation when the lens is placed in the beam line. A small amount of the back reflection from the lens can be used to adjust the angle of the lens. It is convenient to verify that the light passing the lens and reflected by the grating at zeroth-order hits the aperture, installed directly before the second laser.

6. It is recommended to overlap the HeNe laser with $\mathrm{Ti}: \mathrm{Sa}$ laser at reduced output power of the amplifier to the values below $300 \mathrm{~mW}$. The laser power can be reduced by varying the time settings of the second Pockels cell or by blocking the seed beam from entering the regenerative cavity, assuming that the regenerative cavity is optimized for the maximum build up time reduction. Higher precision can be achieved when the lens is removed from its mount and the alignment target (for example a sheet of white paper with markings) is installed after the monochromator at a considerable distance.

7. The last step is to verify the focusing lens alignment, i.e., to check that the transmitted light of HeNe laser is centered at the target, when the focusing lens is installed back to its mount. The longitudinal position (on the optical axis) of the lens can be adjusted using the visible plasma spot generated by the infrared laser in the generation chamber.

\section{A.3 High harmonic generation optimization and characterization}

In this section, the main aspects of the high harmonic generation process are covered. 


\section{Phase matching}

To ensure an efficient conversion of the fundamental near-infrared laser light into high order harmonics, proper phase-matching conditions must be fulfilled. As previously discussed, in the used HHG scheme with the gas filled capillary, the phase-matching conditions for the fixed generation geometry, including the focal parameters and the interaction length, are controlled using the gas pressure optimization. The phase matching is achieved when the free electron dispersion possessing anomalous behavior is balanced with the positive dispersion of the neutral atoms by tuning the gas pressure. Figure A.3 shows the experimental results for phase-matching optimization where the achieved intensities of the harmonic orders are plotted as a function of the gas pressure. Note that the gas pressure inside the capillary can not be directly measured, but the pressure of the gas supply above the generation chamber can provide a reasonable reference when the capillary apertures are relatively small, hence suppressing the excessive gas flow.

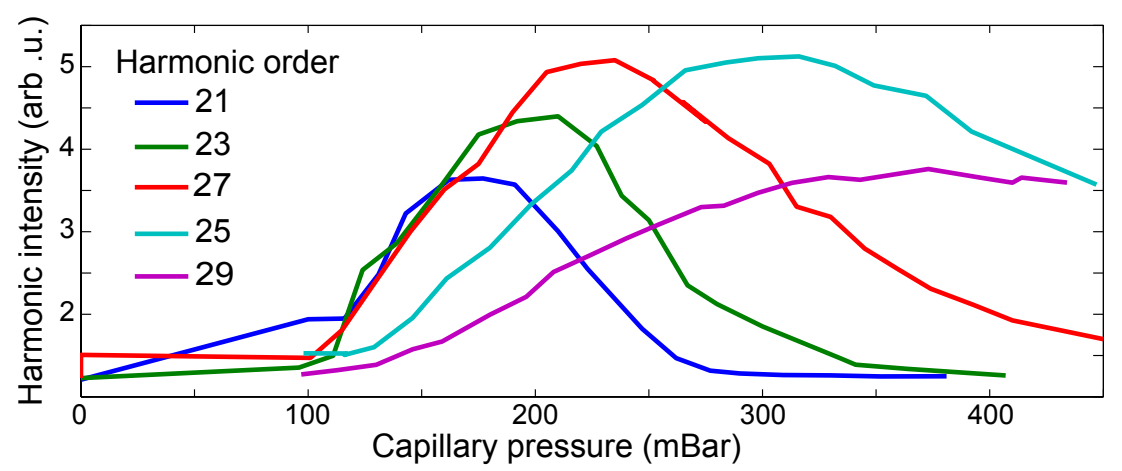

Figure A.3: Pressure dependent phase matching of HHG at fixed laser intensity.

As expected, the maximum intensity for different harmonics is achieved at different capillary pressures. This can be directly attributed to the wavelength dependence of the refractive index of the plasma. The free electron dispersion induces a stronger phase mismatch for higher orders and a higher concentration of the neutrals is required for its compensation. However, this can be accomplished only for a relatively low ionization level. When the proper phase matching conditions are achieved, the lateral position of the focal spot as well as a radial truncation of the fundamental beam can be slightly adjusted to maximize the harmonic yield [205]. 



\section{Bibliography}

[1] Peter Kaiser. Prospective evaluation of visual acuity assessment: a comparison of snellen versus ETDRS charts in clinical practice (An AOS Thesis). Trans. Am. Ophthalmol. Soc., 107:311-324, 2009. Cited on p. 1

[2] M. Born and E. Wolf. Principles of Optics: Electromagnetic Theory of Propagation, Interference and Diffraction of Light. Cambridge University Press, 2000. Cited on p. 2, 9, 35

[3] J. R. Fienup. Reconstruction of an object from the modulus of its Fourier transform. Opt. Lett., 3(1):27-29, 1978. Cited on p. 3, 10, 12

[4] J. R. Fienup. Phase retrieval algorithms: a comparison. Appl. Opt., 21(15):27582769, 1982. Cited on p. 3, 10, 12, 38

[5] Robin Leslie Owen, Enrique Rudiño-Piñera, and Elspeth F. Garman. Experimental determination of the radiation dose limit for cryocooled protein crystals. Proc. Natl. Acad. Sci. U. S. A., 103(13):4912-7, 2006. Cited on p. 3

[6] Henry N. Chapman, Anton Barty, Michael J. Bogan, Sébastien Boutet, Matthias Frank, Stefan P. Hau-Riege, Stefano Marchesini, Bruce W. Woods, Saša Bajt, W. Henry Benner, Richard A. London, Elke Plönjes, Marion Kuhlmann, Rolf Treusch, Stefan Düsterer, Thomas Tschentscher, Jochen R. Schneider, Eberhard Spiller, Thomas Möller, Christoph Bostedt, Matthias Hoener, David A. Shapiro, Keith O. Hodgson, David van der Spoel, Florian Burmeister, Magnus Bergh, Carl Caleman, Gösta Huldt, M. Marvin Seibert, Filipe R. N. C. Maia, Richard W. Lee, Abraham Szöke, Nicusor Timneanu, and Janos Hajdu. Femtosecond diffractive imaging with a soft-X-ray free-electron laser. Nat. Phys., 2(12):839-843, 2006. Cited on p. 3, 4, 15, 17, 36, 38, 45 
[7] Henry N. Chapman, Petra Fromme, Anton Barty, Thomas A. White, Richard A. Kirian, Andrew Aquila, Mark S. Hunter, Joachim Schulz, Daniel P. DePonte, Uwe Weierstall, R. Bruce Doak, Filipe R. N. C. Maia, Andrew V. Martin, Ilme Schlichting, Lukas Lomb, Nicola Coppola, Robert L. Shoeman, Sascha W. Epp, Robert Hartmann, Daniel Rolles, Artem Rudenko, Lutz Foucar, Nils Kimmel, Georg Weidenspointner, Peter Holl, Mengning Liang, Miriam Barthelmess, Carl Caleman, Sébastien Boutet, Michael J. Bogan, Jacek Krzywinski, Christoph Bostedt, Saša Bajt, Lars Gumprecht, Benedikt Rudek, Benjamin Erk, Carlo Schmidt, André Hömke, Christian Reich, Daniel Pietschner, Lothar Strüder, Günter Hauser, Hubert Gorke, Joachim Ullrich, Sven Herrmann, Gerhard Schaller, Florian Schopper, Heike Soltau, Kai-Uwe Kühnel, Marc Messerschmidt, John D. Bozek, Stefan P. Hau-Riege, Matthias Frank, Christina Y. Hampton, Raymond G. Sierra, Dmitri Starodub, Garth J. Williams, Janos Hajdu, Nicusor Timneanu, M. Marvin Seibert, Jakob Andreasson, Andrea Rocker, Olof Jönsson, Martin Svenda, Stephan Stern, Karol Nass, Robert Andritschke, Claus-Dieter Schröter, Faton Krasniqi, Mario Bott, Kevin E. Schmidt, Xiaoyu Wang, Ingo Grotjohann, James M. Holton, Thomas R. M. Barends, Richard Neutze, Stefano Marchesini, Raimund Fromme, Sebastian Schorb, Daniela Rupp, Marcus Adolph, Tais Gorkhover, Inger Andersson, Helmut Hirsemann, Guillaume Potdevin, Heinz Graafsma, Björn Nilsson, and John C. H. Spence. Femtosecond X-ray protein nanocrystallography. Nature, 470(7332):73-77, 2011. Cited on p. 3

[8] Anton Barty, Carl Caleman, Andrew Aquila, Nicusor Timneanu, Lukas Lomb, Thomas A. White, Jakob Andreasson, David Arnlund, Saša Bajt, Thomas R. M. Barends, Miriam Barthelmess, Michael J. Bogan, Christoph Bostedt, John D. Bozek, Ryan Coffee, Nicola Coppola, Jan Davidsson, Daniel P. DePonte, R. Bruce Doak, Tomas Ekeberg, Veit Elser, Sascha W. Epp, Benjamin Erk, Holger Fleckenstein, Lutz Foucar, Petra Fromme, Heinz Graafsma, Lars Gumprecht, Janos Hajdu, Christina Y. Hampton, Robert Hartmann, Andreas Hartmann, Günter Hauser, Helmut Hirsemann, Peter Holl, Mark S. Hunter, Linda Johansson, Stephan Kassemeyer, Nils Kimmel, Richard A. Kirian, Mengning Liang, Filipe R. N. C. Maia, Erik Malmerberg, Stefano Marchesini, Andrew V. Martin, Karol Nass, Richard Neutze, Christian Reich, Daniel Rolles, Benedikt Rudek, Artem Rudenko, Howard Scott, Ilme Schlichting, Joachim Schulz, M. Marvin Seibert, Robert L. Shoe- 
man, Raymond G. Sierra, Heike Soltau, John C. H. Spence, Francesco Stellato, Stephan Stern, Lothar Strüder, Joachim Ullrich, X. Wang, Georg Weidenspointner, Uwe Weierstall, Cornelia B. Wunderer, and Henry N. Chapman. Self-terminating diffraction gates femtosecond X-ray nanocrystallography measurements. Nat. Photonics, 6(1):35-40, 2011. Cited on p. 3, 4

[9] Richard Neutze, Remco Wouts, David Van Der Spoel, and Edgar Weckert. Potential for biomolecular imaging with femtosecond X-ray pulses. Nature, 406:752757, 2000. Cited on p. 3

[10] Marvin Seibert, Tomas Ekeberg, Filipe Maia, Martin Svenda, Jakob Andreasson, Olof Joensson, Dusko Odic, Bianca Iwan, Andrea Rocker, Daniel Westphal, Max Hantke, Daniel P. DePonte, Anton Barty, Joachim Schulz, Lars Gumprecht, Nicola Coppola, Andrew Aquila, Mengning Liang, Thomas A. White, Andrew Martin, Carl Caleman, Stephan Stern, Chantal Abergel, Virginie Seltzer, JeanMichel Claverie, Christoph Bostedt, John D. Bozek, Sebastien Boutet, A. Alan Miahnahri, Marc Messerschmidt, Jacek Krzywinski, Garth Williams, Keith O. Hodgson, Michael J. Bogan, Christina Y. Hampton, Raymond G. Sierra, Dmitri Starodub, Inger Andersson, Sasa Bajt, Miriam Barthelmess, John C. H. Spence, Petra Fromme, Uwe Weierstall, Richard Kirian, Mark Hunter, R. Bruce Doak, Stefano Marchesini, Stefan P. Hau-Riege, Matthias Frank, Robert L. Shoeman, Lukas Lomb, Sascha W Epp, Robert Hartmann, Daniel Rolles, Artem Rudenko, Carlo Schmidt, Lutz Foucar, Nils Kimmel, Peter Holl, Benedikt Rudek, Benjamin Erk, Andre Hoemke, Christian Reich, Daniel Pietschner, Georg Weidenspointner, Lothar Strueder, Guenter Hauser, Hubert Gorke, Joachim Ullrich, Ilme Schlichting, Sven Herrmann, Gerhard Schaller, Florian Schopper, Heike Soltau, Kai-Uwe Kuehnel, Robert Andritschke, Claus-Dieter Schroeter, Faton Krasniqi, Mario Bott, Sebastian Schorb, Daniela Rupp, Marcus Adolph, Tais Gorkhover, Helmut Hirsemann, Guillaume Potdevin, Heinz Graafsma, Bjoern Nilsson, Henry N. Chapman, and Janos Hajdu. Single mimivirus particles intercepted and imaged with an X-ray laser. Nature, 470(7332):78-86, 2011. Cited on p. 3, 14

[11] Lars Redecke, Karol Nass, Daniel P. DePonte, Thomas White, Dirk Rehders, Anton Barty, Francesco Stellato, Mengning Liang, Thomas R. M. Barends, Sébastien Boutet, Garth J. Williams, Marc Messerschmidt, M. Marvin Seibert, Andrew 
Aquila, David Arnlund, Sasa Bajt, Torsten Barth, Michael J. Bogan, Carl Caleman, Tzu-Chiao Chao, R Bruce Doak, Holger Fleckenstein, Matthias Frank, Raimund Fromme, Lorenzo Galli, Ingo Grotjohann, Mark S. Hunter, Linda C. Johansson, Stephan Kassemeyer, Gergely Katona, Richard Kirian, Rudolf Koopmann, Chris Kupitz, Lukas Lomb, Andrew V. Martin, Stefan Mogk, Richard Neutze, Robert L. Shoeman, Jan Steinbrener, Nicusor Timneanu, Dingjie Wang, Uwe Weierstall, Nadia Zatsepin, John C. H. Spence, Petra Fromme, Ilme Schlichting, Michael Duszenko, Christian Betzel, and Henry N. Chapman. Natively inhibited Trypanosoma brucei Cathepsin B structure determined by using an X-ray laser. Science, 339:227-30, 2013. Cited on p. 3

[12] A. McPherson, G. Gibson, H. Jara, U. Johann, T. S. Luk, I. McIntyre, K. Boyer, and C. K. Rhodes. Studies of multiphoton production of vacuum-ultraviolet radiation in the rare gases. J. Opt. Soc. Am. B, 4(4):595 - 601, 1987. Cited on p. 4, 14, 18, 27,37

[13] A. Ravasio, D. Gauthier, F. Maia, M. Billon, J. P. Caumes, D. Garzella, M. Géléoc, O. Gobert, J. F. Hergott, a. M. Pena, H. Perez, B. Carré, E. Bourhis, J. Gierak, A. Madouri, D. Mailly, B. Schiedt, M. Fajardo, J. Gautier, P. Zeitoun, P. H. Bucksbaum, J. Hajdu, and H. Merdji. Single-shot diffractive imaging with a table-top femtosecond soft X-ray laser-harmonics source. Phys. Rev. Lett., 103(2):1-5, 2009. Cited on p. 4, 15, 25, 36, 50, 60

[14] S. Baker, J. S. Robinson, C. A. Haworth, H. Teng, R. A. Smith, C. C. ChirilÄ, M. Lein, J. W. G. Tisch, and J. P. Marangos. Probing Proton Dynamics in Molecules on an Attosecond Time Scale. Science, 312(5772):424-427, apr 2006. Cited on p. 4

[15] Olga Smirnova, Yann Mairesse, Serguei Patchkovskii, Nirit Dudovich, David Villeneuve, Paul Corkum, and Misha Yu Ivanov. High harmonic interferometry of multi-electron dynamics in molecules. Nature, 460(7258):972-977, 2009. Cited on p. 4

[16] R. Cireasa, A. E. Boguslavskiy, B. Pons, M. C. H. Wong, D. Descamps, S. Petit, H. Ruf, N. Thiré, A. Ferré, J. Suarez, J. Higuet, B. E. Schmidt, A. F. Alharbi, F. Légaré, V. Blanchet, B. Fabre, S. Patchkovskii, O. Smirnova, Y. Mairesse, and 
V. R. Bhardwaj. Probing molecular chirality on a sub-femtosecond timescale. Nat. Phys., 11(8):654-658, 2015. Cited on p. 4

[17] L. Miaja-Avila, G. Saathoff, S. Mathias, J. Yin, C. La-o Vorakiat, M. Bauer, M. Aeschlimann, M. M. Murnane, and H. C. Kapteyn. Direct measurement of core-level relaxation dynamics on a surface-adsorbate system using ultrafast Xrays. Conf. Quantum Electron. Laser Sci. - Tech. Dig. Ser., 046101(July):1-4, 2008. Cited on p. 4

[18] Stefan Mathias, Henry C Kapteyn, and Margaret M Murnane. Ultrafast Nonlinear Optics. chapter Ultrafast, pages 149-175. Springer International Publishing, Heidelberg, 2013. Cited on p. 4

[19] M. Hentschel, R. Kienberger, C. Spielmann, G. Reider, N. Milosevic, T. Brabec, P. Corkum, U. Heinzmann, M. Drescher, and F. Krausz. Attosecond metrology. Nature, 414(6863):509-513, 2001. Cited on p. 4

[20] T. Pfeifer, C. Spielmann, and G. Gerber. Femtosecond X-ray science. Reports Prog. Phys., 69(2):443-505, 2006. Cited on p. 4

[21] P. B. Corkum and F. Krausz. Attosecond science. Nat. Phys., 3:381-387, 2007. Cited on p. 4, 49

[22] Ferenc Krausz and Misha Ivanov. Attosecond physics. Rev. Mod. Phys., 81(1):163234, 2009. Cited on p. 4

[23] T. Popmintchev, M.-C. Chen, D. Popmintchev, P. Arpin, S. Brown, S. Alisauskas, G. Andriukaitis, T. Balciunas, O. D. Mucke, A. Pugzlys, A. Baltuska, B. Shim, S. E. Schrauth, A. Gaeta, C. Hernandez-Garcia, L. Plaja, A. Becker, A. JaronBecker, M. M. Murnane, and H. C. Kapteyn. Bright Coherent Ultrahigh Harmonics in the keV X-ray Regime from Mid-Infrared Femtosecond Lasers. Science, 336(6086):1287-1291, 2012. Cited on p. 4, 19

[24] Maciej Salieres, Pascal and L'Huillier, Anne and Lewenstein. Coherence control of High-order harmonocs. Phys. Rev. Lett., 74(19):3776, 1995. Cited on p. 4, 21

[25] T. Ditmire, E. T. Gumbrell, R. Smith, J. W. G. Tisch, D. D. Meyerhofer, and M. Hutchinson. Spatial Coherence Measurement of Soft X-Ray Radiation Pro- 
duced by High Order Harmonic Generation. Phys. Rev. Lett., 77(23):4756 - 4759, 1996. Cited on p. 4

[26] Xinkui He, M. Miranda, J. Schwenke, O. Guilbaud, T. Ruchon, C. Heyl, E. Georgadiou, R. Rakowski, a. Persson, M. B. Gaarde, and a. L'Huillier. Spatial and spectral properties of the high-order harmonic emission in argon for seeding applications. Phys. Rev. A - At. Mol. Opt. Phys., 79(6):1-7, 2009. Cited on p. 4

[27] Avner Fleischer, Ofer Kfir, Tzvi Diskin, Pavel Sidorenko, and Oren Cohen. Spin angular momentum and tunable polarization in high-harmonic generation. Nat. Photonics, 8(7):543-549, 2014. Cited on p. 4, 5, 50, 72, 83, 84

[28] A. Ferré, C. Handschin, M. Dumergue, F. Burgy, A. Comby, D. Descamps, B. Fabre, G. A. Garcia, R. Géneaux, L. Merceron, E. Mével, L. Nahon, S. Petit, B. Pons, D. Staedter, S. Weber, T. Ruchon, V. Blanchet, and Y. Mairesse. A table-top ultrashort light source in the extreme ultraviolet for circular dichroism experiments. Nat. Photonics, 9(2):93-98, 2014. Cited on p. 4, 50, 72

[29] G. Lambert, B. Vodungbo, J. Gautier, B. Mahieu, V. Malka, S. Sebban, P. Zeitoun, J. Luning, J. Perron, A. Andreev, S. Stremoukhov, F. Ardana-Lamas, A. Dax, C. P. Hauri, A. Sardinha, and M. Fajardo. Towards enabling femtosecond helicitydependent spectroscopy with high-harmonic sources. Nat. Commun., 6:6167, 2015. Cited on p. 4, 50, 72

[30] Daniel D. Hickstein, Franklin J. Dollar, Patrik Grychtol, Jennifer L. Ellis, Ronny Knut, Carlos Hernández-García, Dmitriy Zusin, Christian Gentry, Justin M. Shaw, Tingting Fan, Kevin M. Dorney, Andreas Becker, Agnieszka Jaro̊̊-Becker, Henry C. Kapteyn, Margaret M. Murnane, and Charles G. Durfee. Non-collinear generation of angularly isolated circularly polarized high harmonics. Nat. Photonics, pages 1-10, 2015. Cited on p. 4, 50, 72, 84, 85

[31] Kun Zhao, Qi Zhang, Michael Chini, Yi Wu, Xiaowei Wang, and Zenghu Chang. Tailoring a 67 attosecond pulse through advantageous phase mismatch. Opt. Lett., 37(18):3891-3893, 2012. Cited on p. 4

[32] Steffen Hädrich, Arno Klenke, Jan Rothhardt, Manuel Krebs, Armin Hoffmann, Oleg Pronin, Vladimir Pervak, Jens Limpert, and Andreas Tünnermann. High pho- 
ton flux table-top coherent extreme-ultraviolet source. Nat. Photonics, 8(10):779783, 2014. Cited on p. 5, 24

[33] H. Eichmann, A. Egbert, and S. Nolte. Polarization-Dependent High-Order TwoColor Mixing. Phys. Rev. A, 51(5):3414-3417, 1995. Cited on p. 5, 50, 84, 85

[34] Ofer Kfir, Patrik Grychtol, Emrah Turgut, Ronny Knut, Dmitriy Zusin, Dimitar Popmintchev, Tenio Popmintchev, Hans Nembach, Justin M. Shaw, Avner Fleischer, Henry Kapteyn, Margaret Murnane, and Oren Cohen. Generation of bright phase-matched circularly-polarized extreme ultraviolet high harmonics. Nat. Photonics, 9(2):99-105, 2014. Cited on p. 5, 50, 56, 72, 84

[35] Tingting Fan, Patrik Grychtol, Ronny Knut, Carlos Hernandez-Garcia, Daniel D. Hickstein, Dmitriy Zusin, Christian Gentry, Franklin J. Dollar, Christopher A. Mancuso, Craig W. Hogle, Ofer Kfir, Dominik Legut, Karel Carva, Jennifer L. Ellis, Kevin M. Dorney, Cong Chen, Oleg G. Shpyrko, Eric E. Fullerton, Oren Cohen, Peter M. Oppeneer, Dejan B. Milosevic, Andreas Becker, Agnieszka A. Jaron-Becker, Tenio Popmintchev, Margaret M. Murnane, and Henry C. Kapteyn. Bright circularly polarized soft X-ray high harmonics for X-ray magnetic circular dichroism. Proc. Natl. Acad. Sci., 112(46):14206-11, 2015. Cited on p. 5, 72, 84, 85

[36] A. Lewis, M. Isaacson, A. Harootunian, and A. Muray. Development of a $500 \AA$ spatial resolution light microscope. Ultramicroscopy, 13(3):227-231, 1984. Cited on p. 8

[37] U. Dürig, D. W. Pohl, and F. Rohner. Near-field optical-scanning microscopy. J. Appl. Phys., 59(10):3318-3327, 1986. Cited on p. 8

[38] Rolf Erni, Marta D. Rossell, Christian Kisielowski, and Ulrich Dahmen. Atomicresolution imaging with a sub-50-pm electron probe. Phys. Rev. Lett., 102(9):1-4, 2009. Cited on p. 8

[39] A. Snigirev, V. Kohn, I. Snigireva, and B. Lengeler. A compound refractive lens for focusing high-energy X-rays. Nature, 384(6604):49-51, nov 1996. Cited on p. 8 
[40] Boris Benner, Bruno Lengeler, Christian Schroer, Johannes Tu, Matthias Richwin, Anatoly Snigirev, and Irina Snigireva. Imaging by parabolic refractive lenses in the hard X-ray range. J. Synchrotron Radiat., 6(6):1153-1167., 1999. Cited on p. 8

[41] F. Marschall, A. Last, M. Simon, M. Kluge, V. Nazmov, H. Vogt, M. Ogurreck, I. Greving, and J. Mohr. X-ray Full Field Microscopy at $30 \mathrm{keV}$. J. Phys. Conf. Ser., 499(1):12007, 2014. Cited on p. 8

[42] M. Berglund, L. Rymell, and H. M. Hertz. Compact water-window transmission X-ray microscopy. 197(March):268-273, 2000. Cited on p. 9

[43] Diling Zhu, Manuel Guizar-Sicairos, Benny Wu, Andreas Scherz, Yves Acremann, Tolek Tyliszczak, Peter Fischer, Nina Friedenberger, Katharina Ollefs, Michael Farle, James R. Fienup, and Joachim Stohr. High-resolution X-ray lensless imaging by differential holographic encoding. Phys. Rev. Lett., 105(4):1-4, 2010. Cited on p. 9,16

[44] Kahraman Keskinbora, Anna-lena Robisch, Marcel Mayer, Umut T Sanli, Corinne Grévent, Christian Wolter, Markus Weigand, Adriana Szeghalmi, Mato Knez, Tim Salditt, and Gisela Schütz. Multilayer Fresnel zone plates for high energy radiation resolve $21 \mathrm{~nm}$ features at $1.2 \mathrm{keV} .22(15): 301-309,2014$. Cited on p. 9

[45] Anne Sakdinawat and David Attwood. Nanoscale X-ray imaging. Nat. Photonics, 4(12):840-848, 2010. Cited on p. 9

[46] Jianwei Miao, Tetsuya Ishikawa, Ian K Robinson, and Margaret M Murnane. Beyond crystallography: Diffractive imaging using coherent x-ray light sources. Science, 348(6234):530-535, apr 2015. Cited on p. 9, 50, 52

[47] William Lawrence Bragg. The structure of some crystals as indicated by their diffraction of X-rays. Proc. R. Soc. London A Math. Phys. Eng. Sci., 89(610):248277, 1913. Cited on p. 9

[48] W. Friedrich, P. Knipping, and M. Laue. Interferenzerscheinungen bei Röntgenstrahlen. Ann. Phys., 346(10):971-988, 1913. Cited on p. 9

[49] R. W. Gerchberg. A practical algorithm for the determination of phase from image and diffraction plane pictures. Optik (Stuttg)., 35:237, 1972. Cited on p. 10 
[50] Veit Elser. Phase retrieval by iterated projections. J. Opt. Soc. Am. A. Opt. Image Sci. Vis., 20(1):40-55, 2003. Cited on p. 12

[51] A. T. Brünger, P. D. Adams, G. M. Clore, W. L. DeLano, P. Gros, R. W. GrosseKunstleve, J. S. Jiang, J. Kuszewski, M. Nilges, N. S. Pannu, R. J. Read, L. M. Rice, T. Simonson, and G. L. Warren. Crystallography \&amp; NMR System: A New Software Suite for Macromolecular Structure Determination. Acta Crystallogr. Sect. D Biol. Crystallogr., 54(5):905-921, 1998. Cited on p. 12

[52] Russell Luke. Relaxed averaged alternating reflections for diffraction imaging. Inverse Probl., 21(1):37-50, 2005. Cited on p. 12, 38, 52

[53] Jose A. Rodriguez, Rui Xu, Chien Chun Chen, Yunfei Zou, and Jianwei Miao. Oversampling smoothness: An effective algorithm for phase retrieval of noisy diffraction intensities. J. Appl. Crystallogr., 46(2):312-318, 2013. Cited on p. 12

[54] J. M. Rodenburg, A. C. Hurst, A. G. Cullis, B. R. Dobson, F. Pfeiffer, O. Bunk, C. David, K. Jefimovs, and I. Johnson. Hard-X-Ray Lensless Imaging of Extended Objects. 034801(January):1-4, 2007. Cited on p. 12, 13

[55] Brian Abbey, Keith A Nugent, Garth J Williams, Jesse N Clark, Andrew G Peele, Mark A Pfeifer, Martin de Jonge, and Ian McNulty. Keyhole coherent diffractive imaging. Nat. Phys., 4(5):394-398, 2008. Cited on p. 12

[56] Bosheng Zhang, M.D. Seaberg, Daniel E. Adams, D.F. Gardner, E.R. Shanblatt, J.M. Shaw, W. Chao, E.M. Gullikson, F. Salmassi, H.C. Kapteyn, and M.M. Murnane. Full field tabletop EUV coherent diffractive imaging in a transmission geometry. Opt. Express, 21(19):839-843, 2013. Cited on p. 12, 36, 60

[57] E. B. Malm, N. C. Monserud, C. G. Brown, P. W. Wachulak, H. Xu, G. Balakrishnan, W. Chao, E. Anderson, and M. C. Marconi. Tabletop single-shot extreme ultraviolet Fourier transform holography of an extended object. Opt. Express, 21(8):9959-9966, 2013. Cited on p. 12

[58] J. Miao, D. Sayre, and H. N. Chapman. Phase retrieval from the magnitude of the Fourier transforms of nonperiodic objects. J. Opt. Soc. Am. A, 15(6):1662, 1998. Cited on p. 13 
[59] Jianwei Miao, Tetsuya Ishikawa, Erik H. Anderson, and Keith O. Hodgson. Phase retrieval of diffraction patterns from noncrystalline samples using the oversampling method. Phys. Rev. B, 67(17):174104, 2003. Cited on p. 13, 37, 60, 65, 74

[60] Pierre Thibault, Martin Dierolf, Andreas Menzel, Oliver Bunk, Christian David, and Franz Pfeiffer. High-Resolution Scanning X-ray Diffraction Microscopy. Science, 321(5887):379-382, jul 2008. Cited on p. 13, 36

[61] K. Giewekemeyer, M. Beckers, T. Gorniak, M. Grunze, T. Salditt, and A. Rosenhahn. Ptychographic coherent x-ray diffractive imaging in the water window. Opt. Express, 19(2):1037-1050, 2011. Cited on p. 13, 14

[62] David A. Shapiro, Young-Sang Yu, Tolek Tyliszczak, Jordi Cabana, Rich Celestre, Weilun Chao, Konstantin Kaznatcheev, A. L. David Kilcoyne, Filipe Maia, Stefano Marchesini, Y. Shirley Meng, Tony Warwick, Lee Lisheng Yang, and Howard A. Padmore. Chemical composition mapping with nanometre resolution by soft X-ray microscopy. Nat. Photonics, 8(10):765-769, 2014. Cited on p. 13, 14

[63] Matthew D Seaberg, Bosheng Zhang, Dennis F Gardner, Elisabeth R Shanblatt, Margaret M Murnane, Henry C Kapteyn, and Daniel E Adams. Tabletop nanometer extreme ultraviolet imaging in an extended reflection mode using coherent Fresnel ptychography:supplementary material. Optica, 1(1):39, 2014. Cited on p. 13, 15

[64] Marco Stockmar, Irene Zanette, Martin Dierolf, Bjoern Enders, Richard Clare, Franz Pfeiffer, Peter Cloetens, Anne Bonnin, and Pierre Thibault. X-Ray NearField Ptychography for Optically Thick Specimens. Phys. Rev. Appl., 3(1):014005, 2015. Cited on p. 13

[65] Yoshinori Nishino, Yukio Takahashi, Naoko Imamoto, Tetsuya Ishikawa, and Kazuhiro Maeshima. Three-dimensional visualization of a human chromosome using coherent X-ray diffraction. Phys. Rev. Lett., 102(1):8-11, 2009. Cited on p. 14

[66] Samuel Flewett, Stefan Schaffert, Jyoti Mohanty, Erik Guehrs, Jan Geilhufe, Christian M. Günther, Bastian Pfau, and Stefan Eisebitt. Method for Single-Shot Coherent Diffractive Imaging of Magnetic Domains. Phys. Rev. Lett., 108(22):223902, 2012. Cited on p. 14,36 
[67] Jianwei Miao, Pambos Charalambous, Janos Kirz, and David Sayre. Extending the methodology of X-ray crystallography to allow imaging of micrometre-sized non-crystalline specimens. Nature, 400(6742):342-344, 1999. Cited on p. 13, 36, 61

[68] Huaidong Jiang, Rui Xu, Chien-Chun Chen, Wenge Yang, Jiadong Fan, Xutang Tao, Changyong Song, Yoshiki Kohmura, Tiqiao Xiao, Yong Wang, Yingwei Fei, Tetsuya Ishikawa, Wendy L Mao, and Jianwei Miao. Three-Dimensional Coherent X-Ray Diffraction Imaging of Molten Iron in Mantle Olivine at Nanoscale Resolution. Phys. Rev. Lett., 110(20):205501, 2013. Cited on p. 14

[69] Changyong Song, Raymond Bergstrom, Damien Ramunno-Johnson, Huaidong Jiang, David Paterson, Martin D. De Jonge, Ian McNulty, Jooyoung Lee, Kang L. Wang, and Jianwei Miao. Nanoscale imaging of buried structures with elemental specificity using resonant X-ray diffraction microscopy. Phys. Rev. Lett., 100(2):18-21, 2008. Cited on p. 14

[70] Chan Kim, Yoonhee Kim, Changyong Song, Sang Soo Kim, Sunam Kim, Hyon Chol Kang, Yeukuang Hwu, Ku-Ding Tsuei, Keng San Liang, and Do Young Noh. Resolution enhancement in coherent x-ray diffraction imaging by overcoming instrumental noise. Opt. Express, 22(23):29161, 2014. Cited on p. 14

[71] W. Eberhardt S. Eisebitt, J. Lüning, W. F. Schlotter, M. Lörgen, O. Hellwig and J. Stöhr. Lensless imaging of magnetic nanostructures by X-ray. Nature, 432:885888,2004 . Cited on p. $14,36,50,87,88$

[72] Joshua J. Turner, Xiaojing Huang, Oleg Krupin, Keoki A. Seu, Daniel Parks, Stephen Kevan, Enju Lima, Kim Kisslinger, Ian McNulty, Richard Gambino, Stephane Mangin, Sujoy Roy, and Peter Fischer. X-Ray Diffraction Microscopy of Magnetic Structures. Phys. Rev. Lett., 107(3):033904, 2011. Cited on p. 14

[73] A. Tripathi, J. Mohanty, S. H. Dietze, O. G. Shpyrko, E. Shipton, E. E. Fullerton, S. S. Kim, and I. McNulty. Dichroic coherent diffractive imaging. Proc. Natl. Acad. Sci., 108(33):13393-13398, 2011. Cited on p. 14, 50

[74] Mark A. Pfeifer, Garth J. Williams, Ivan Vartanyants, Ross Harder, and Ian K. 
Robinson. Three-dimensional mapping of a deformation field inside a nanocrystal. Nature, 442(7098):63-6, 2006. Cited on p. 14, 27, 36

[75] Ian Robinson and Ross Harder. Coherent X-ray diffraction imaging of strain at the nanoscale. Nat. Mater., 8(4):291-298, 2009. Cited on p. 14

[76] Wenge Yang, Xiaojing Huang, Ross Harder, Jesse N Clark, Ian K Robinson, and Ho-kwang Mao. Coherent diffraction imaging of nanoscale strain evolution in a single crystal under high pressure. Nat. Commun., 4(Cxdi):1680, 2013. Cited on p. 14

[77] Yukio Takahashi, Yoshinori Nishino, Ryosuke Tsutsumi, Nobuyuki Zettsu, Eiichiro Matsubara, Kazuto Yamauchi, and Tetsuya Ishikawa. High-resolution projection image reconstruction of thick objects by hard x-ray diffraction microscopy. Phys. Rev. B, 82(21):214102, 2010. Cited on p. 14

[78] Kevin S. Raines, Sara Salha, Richard L. Sandberg, Huaidong Jiang, Jose A. Rodríguez, Benjamin P. Fahimian, Henry C. Kapteyn, Jincheng Du, and Jianwei Miao. Three-dimensional structure determination from a single view. Nature, 463(7278):214-217, 2010. Cited on p. 14, 43

[79] Huaidong Jiang, Changyong Song, and CC Chen. Quantitative 3D imaging of whole, unstained cells by using X-ray diffraction microscopy. Proc. Natl. Acad. Sci. U. S. A., 107(25):11234-11239, 2010. Cited on p. 14

[80] Anton Barty, Sébastien Boutet, Michael J. Bogan, Stefan Hau-Riege, Stefano Marchesini, Klaus Sokolowski-Tinten, Nikola Stojanovic, Ra'anan Tobey, Henri Ehrke, Andrea Cavalleri, Stefan Düsterer, Matthias Frank, Saša Bajt, Bruce W. Woods, M. Marvin Seibert, Janos Hajdu, Rolf Treusch, and Henry N. Chapman. Ultrafast single-shot diffraction imaging of nanoscale dynamics. Nat. Photonics, 2(9):578-578, 2008. Cited on p. 15, 17

[81] J. Geilhufe, C. Tieg, B. Pfau, C. M. Günther, E. Guehrs, S. Schaffert, and S. Eisebitt. Extracting depth information of 3-dimensional structures from a singleview X-ray Fourier-transform hologram. Opt. Express, 22(21):24959-69, 2014. Cited on p. 15 
[82] Dennis F. Gardner, Bosheng Zhang, Matthew D. Seaberg, Leigh S. Martin, Daniel E. Adams, Farhad Salmassi, Eric Gullikson, Henry Kapteyn, and Margaret Murnane. High numerical aperture reflection mode coherent diffraction microscopy using off-axis apertured illumination. Opt. Express, 20(17):19050, 2012. Cited on p. 15

[83] Michael Zürch, Christian Kern, and Christian Spielmann. XUV coherent diffraction imaging in reflection geometry with low numerical aperture. Opt. Express, 21(18):21131-21147, 2013. Cited on p. 15

[84] Bosheng Zhang, Dennis F. Gardner, Matthew D. Seaberg, Elisabeth R. Shanblatt, Henry C. Kapteyn, Margaret M. Murnane, and Daniel E. Adams. High contrast 3D imaging of surfaces near the wavelength limit using tabletop EUV ptychography. Ultramicroscopy, 158:98-104, 2015. Cited on p. 15

[85] Bo Huang, Mark Bates, and Xiaowei Zhuang. Super resolution fluorescence microscopy. Annu. Rev. Biochem., 78:993-1016, 2010. Cited on p. 15

[86] Magnus Bergh, Gösta Huldt, Nicusor Tîmneanu, Filipe R N C Maia, and Janos Hajdu. Feasibility of imaging living cells at subnanometer resolutions by ultrafast X-ray diffraction. Q. Rev. Biophys., 41(3-4):181-204, 2008. Cited on p. 15

[87] W. F. Schlotter, R. Rick, K. Chen, A. Scherz, J. Stöhr, J. Lüning, S. Eisebitt, Ch. Günther, W. Eberhardt, I. Mcnulty, W. F. Schlotter, R. Rick, and K. Chen. Multiple reference Fourier transform holography with soft x rays. Appl. Phzs. Lett., 89(163112), 2006. Cited on p. 16

[88] J. Geilhufe, B. Pfau, M. Schneider, F. Büttner, C. M. Günther, S. Werner, S. Schaffert, E. Guehrs, S. Frömmel, M. Kläui, and S. Eisebitt. Monolithic focused reference beam X-ray holography. Nat. Commun., 5:1-6, 2014. Cited on p. 16

[89] Manuel Guizar-Sicairos and James R Fienup. Holography with extended reference by autocorrelation linear differential operation. Opt. Express, 15(26):1759217612, 2007. Cited on p. 16

[90] D. Gauthier, M. Guizar-Sicairos, X. Ge, W. Boutu, B. Carré, J. R. Fienup, and H. Merdji. Single-shot Femtosecond X-Ray Holography Using Extended References. Phys. Rev. Lett., 105(9):093901, 2010. Cited on p. 16, 25 
[91] S. Eisebitt, M. Loergen, W. Eberhardt, J. Luening, S. Andrews, and J. Stoehr. Scalable approach for lensless imaging at x-ray wavelengths. Appl. Phys. Lett., 84(17):3373, 2004. Cited on p. 16

[92] R. L. Sandberg, D. A. Raymondson, C. La-o vorakiat, A. Paul, K. S. Raines, J. Miao, M. M. Murnane, H. C. Kapteyn, and W. F. Schlotter. Tabletop soft-Xray Fourier transform holography with $50 \mathrm{~nm}$ resolution. Opt. Lett., 34(11):1618, 2009. Cited on p. 16, 25, 60, 61

[93] R. L. Sandberg, D. A. Raymondson, William F. Schlotter, Kevin Raines, Chan La-O-Vorakiat, Ariel Paul, Margaret M. Murnane, Henry C. Kapteyn, and Jianwei Miao. Near diffraction limited coherent diffractive imaging with tabletop soft x-ray sources. J. Phys. Conf. Ser., 186:12058, 2009. Cited on p. 16

[94] Zhirong Huang. Brightness and Coherence of Synchrotron Radiation and FELs. Int. Part. Accel. Conf., page 16, 2013. Cited on p. 17

[95] Miltcho B Danailov, Filippo Bencivenga, Flavio Capotondi, Francesco Casolari, Paolo Cinquegrana, Alexander Demidovich, Erika Giangrisostomi, Maya P Kiskinova, Gabor Kurdi, Michele Manfredda, Claudio Masciovecchio, Riccardo Mincigrucci, Ivaylo P Nikolov, Emanuele Pedersoli, Emiliano Principi, and Paolo Sigalotti. Towards jitter-free pump-probe measurements at seeded free electron laser facilities. Opt. Express, 22(11):12869-12879, 2014. Cited on p. 17

[96] P. A. Franken, A. E. Hill, C. W. Peters, and G. Weinreich. Generation of optical harmonics. Phys. Rev. Lett., 7(4):118-119, 1961. Cited on p. 17

[97] J. Reintjes, R. C. Eckardt, C. Y. She, N. E. Karangelen, R. C. Elton, and R. A. Andrews. Generation of coherent radiation at $53.2 \mathrm{~nm}$ by fifth-harmonic conversion. Phys. Rev. Lett., 37(23):1540-1543, 1976. Cited on p. 18

[98] M. Ferray, A. L'Huillier, X. F. Li, L. A. Lompre, G. Mainfray, and C. Manus. Multiple-harmonic conversion of $1064 \mathrm{~nm}$ radiation in rare gases. J. Phys. B At. Mol. Opt. Phys., 21(3):L31, 1988. Cited on p. 18

[99] Jeffrey Krause, Kenneth Schafer, and Kenneth Kulander. High-order harmonic generation from atoms and ions in the high intensity regime. Phys. Rev. Lett., 68(24):3535-3538, 1992. Cited on p. 19 
[100] P. B. Corkum. Plasma perspective on strong field multiphoton ionization. Phys. Rev. Lett., 71(13):1994-1997, 1993. Cited on p. 20

[101] P. B. Corkum, N. H. Burnett, and F. Brunel. Above-threshold ionization in the long-wavelength limit. Phys. Rev. Lett., 62(11):1259-1262, 1989. Cited on p. 20

[102] V. Strelkov. Role of autoionizing state in resonant high-order harmonic generation and attosecond pulse production. Phys. Rev. Lett., 104(12):26-29, 2010. Cited on p. 22

[103] Ivan Christov, Margaret Murnane, and Henry Kapteyn. High-Harmonic Generation of Attosecond Pulses in the "Single-Cycle" Regime. Phys. Rev. Lett., 78(7):12511254, 1997. Cited on p. 22

[104] Philippe Balcou, Pascal Sali ‘eres, Anne L'Huillier, and Maciej Lewenstein. Generalized phase-matching conditions for high harmonics: The role of field-gradient forces. Phys. Rev. A, 55(4):3204-3210, 1997. Cited on p. 23

[105] Henry C. Kapteyn Andy Rundquist, Charles G. Durfee III, Zenghu Chang, Catherine Herne, Sterling Backus, Margaret Murnane. Phase-Matched Generation of Coherent Soft X-rays. Science, 280(5368):1412-1415, 1998. Cited on p. 23, 24, 78

[106] K. C. L'Huillier, A. and Schafer, K. J. and Kulander. Higher-order harmonic generation in xenon at $1064 \mathrm{~nm}$ : The role of phase matching. Phys. Rev. Lett., 66(17):2200-2203, 1991. Cited on p. 23

[107] Tenio Popmintchev, M.C. Ming-Chang Chen, Oren Cohen, Michael E. Grisham, Jorge J. Rocca, Margaret M. Murnane, and Henry C. Kapteyn. Extended phase matching of high harmonics driven by mid-infrared light. Opt. Lett., 33(18):21282130, 2008. Cited on p. 23, 79, 82

[108] Charles Durfee, Andy Rundquist, Sterling Backus, Catherine Herne, Margaret Murnane, and Henry Kapteyn. Phase Matching of High-Order Harmonics in Hollow Waveguides. Phys. Rev. Lett., 83(11):2187-2190, 1999. Cited on p. 23, 24

[109] Randy a Bartels, Ariel Paul, Hans Green, Henry C Kapteyn, Margaret M Murnane, Sterling Backus, Ivan P Christov, Yanwei Liu, David Attwood, and Chris Jacobsen. 
Generation of spatially coherent light at extreme ultraviolet wavelengths. Science, 297(5580):376-378, 2002. Cited on p. 24

[110] M. Chen, M. R. Gerrity, S. Backus, T. Popmintchev, X. Zhou, P. Arpin, H. C. Kapteyn, and M. M. Murnane. Spatially coherent, phase matched, high-order harmonic EUV beams at $50 \mathrm{kHz} .17(20): 9277-9283,2009$. Cited on p. 24

[111] Yusuke Tamaki, Jiro Itatani, Yutaka Nagata, Minoru Obara, and Katsumi Midorikawa. Highly Efficient, Phase-Matched High-Harmonic Generation by a SelfGuided Laser Beam. Phys. Rev. Lett., 82(7):1422-1425, 1999. Cited on p. 24, 91

[112] Richard L. Sandberg, Ariel Paul, Daisy A. Raymondson, Steffen Hädrich, David M. Gaudiosi, Jim Holtsnider, Ra'anan I. Tobey, Oren Cohen, Margaret M. Murnane, Henry C. Kapteyn, Changyong Song, Jianwei Miao, Yanwei Liu, and Farhad Salmassi. Lensless Diffractive Imaging Using Tabletop Coherent HighHarmonic Soft-X-Ray Beams. Phys. Rev. Lett., 99(9):098103, 2007. Cited on p. $24,25,36,60,61$

[113] Richard Lunt Sandberg. Closing the Gap to the Diffraction Limit: Near Wavelength Limited Tabletop Soft X-Ray Coherent Diffractive Imaging. Dissertation, University of Colorado, 2009. Cited on p. 24

[114] Richard L. Sandberg, Changyong Song, Przemyslaw W. Wachulak, Daisy Raymondson, Ariel Paul, Bagrat Amirbekian, Edwin Lee, Anne E. Sakdinawat, Chan La-O-Vorakiat, Mario C. Marconi, Carmen S. Menoni, Margaret M. Murnane, Jorge J. Rocca, Henry C. Kapteyn, and Jianwei Miao. High numerical aperture tabletop soft x-ray diffraction microscopy with 70-nm resolution. Proc. Natl. Acad. Sci. U. S. A., 105(1):24-27, 2008. Cited on p. 24, 60

[115] Bo Chen, Ruben A. Dilanian, Sven Teichmann, Brian Abbey, Andrew G. Peele, Garth J. Williams, Peter Hannaford, Lap Van Dao, Harry M. Quiney, and Keith A. Nugent. Multiple wavelength diffractive imaging. Phys. Rev. A, 79(2):023809, 2009. Cited on p. 25,60

[116] Brian Abbey, Lachlan W. Whitehead, Harry M. Quiney, David J. Vine, Guido A. Cadenazzi, Clare A. Henderson, Keith A. Nugent, Eugeniu Balaur, Corey T. 
Putkunz, Andrew G. Peele, Garth J. Williams, and I. McNulty. Lensless imaging using broadband X-ray sources. Nat Phot., 5(7):420-424, 2011. Cited on p. 25, 60

[117] Matthew D. Seaberg, D. Adams, Ethan L. Townsend, Daisy a. Raymondson, William F. Schlotter, Yanwei Liu, Carmen S. Menoni, Lu Rong, Chien-Chun Chen, Jianwei Miao, Henry C. Kapteyn, and Margaret M. Murnane. Ultrahigh $22 \mathrm{~nm}$ resolution coherent diffractive imaging using a desktop $13 \mathrm{~nm}$ high harmonic source. Opt. Express, 19(23):22470, 2011. Cited on p. 25, 36, 60, 76

[118] M. Schultze, E. Goulielmakis, M. Uiberacker, M. Hofstetter, J. Kim, D. Kim, F. Krausz, and U. Kleineberg. Powerful 170-attosecond XUV pulses generated with few-cycle laser pulses and broadband multilayer optics. New J. Phys., 9(243), 2007. Cited on p. 27

[119] M. Hutley. Diffraction Gratings. Academic Press Inc. Cited on p. 27

[120] B. Schäfer and K. Mann. Investigation of the propagation characteristics of excimer lasers using a HartmannâShack sensor. Rev. Sci. Instrum., 71:2663-2668, 2000. Cited on p. 28

[121] B. Schäfer, M. Lübbecke, and K. Mann. Hartmann-Shack wave front measurements for real time determination of laser beam propagation parameters. Rev. Sci. Instrum., 77(053103), 2006. Cited on p. 28

[122] Bernhard Flöter, Pavle Juranić, Svea Kapitzki, Barbara Keitel, Klaus Mann, Elke Plönjes, Bernd Schäfer, and Kai Tiedtke. EUV Hartmann sensor for wavefront measurements at the Free-electron LASer in Hamburg. New J. Phys., 12(8):83015, 2010. Cited on p. 28, 29

[123] B. Flöter, P. Juranić, P. Großmann, S. Kapitzki, B. Keitel, K. Mann, Plönjes, Schäfer B. E., and K. Tiedtke. Beam parameters of FLASH beamline BL1 from Hartmann wavefront measurements. Nucl. Instruments Methods Phys. Res. Sect. A, 635:S108--S112, 2011. Cited on p. 28, 29

[124] B. L. Henke, E. M. Gullikson, and J. C. Davis. X-Ray Interactions: Photoabsorption, Scattering, Transmission, and Reflection at $\mathrm{E}=50-30,000 \mathrm{eV}, \mathrm{Z}=1-92$. At. data Nucl. data tables, 54:181-342, 1993. Cited on p. 28, 40 
[125] B. Flöter. Strahlcharakterisierung von Freie-Elektronen-Lasern im weichen Röntgen-Spektralbereich. Phd thesis, Georg-August university of Göttingen, 2012. Cited on p. 29

[126] D. Attwood. Soft x-rays and extreme ultraviolet radiation. Cambridge University Press, 1999. Cited on p. 30

[127] E. Hecht. Optics. Pearson Education Limited, 2014. Cited on p. 30

[128] Lasers and laser-related equipment - Test methods for laser beam widths, divergence angle and beam propagation ratio - Part 1: Stigmatic and simple astigmatic beams. ISO 11146-1, 2003. Cited on p. 31

[129] B. Eppich. Die Charakterisierung von Strahlungsfeldern mit der Wigner-Verteilung und deren Messung. Phd thesis, Technical university of Berlin, 1998. Cited on p. 31,33

[130] A. E. Siegman. ABCD-matrix elements for a curved diffraction grating. J. Opt. Soc. Am. A, pages 1793-1793, 1985. Cited on p. 33

[131] H. F. Schouten, N. Kuzmin, G. Dubois, T. D. Visser, G. Gbur, P. F A Alkemade, H. Blok, G. W. 'T Hooft, D. Lenstra, and E. R. Eliel. Plasmon-assisted two-slit transmission: Young's experiment revisited. Phys. Rev. Lett., 94(5):1-4, 2005. Cited on p. 36

[132] Chris Mack. Fundamental Principles of Optical Lithography: The Science of Microfabrication. Wiley, 2007. Cited on p. 36

[133] A. Poonawala. Mask design for single and double exposure optical microlithography: an inverse imaging approach. 2007. Cited on p. 36

[134] K .A. Goldberg, E. Tejnil, J. Bokor, G. D. Kubiac, and D. R. Kania. A 3-D numerical study of pinhole diffraction to predict the accuracy of EUV point diffraction interferometry. Extrem. Ultrav. Lithogr., 4:133-137, 1996. Cited on p. 36

[135] A. R. Hare and G. R. Morrison. Near-field Soft X-ray Diffraction Modelled by the Multislice Method. J. Mod. Opt., 41(1):31-48, 1994. Cited on p. 36 
[136] Alexei Erko, Mourad Idir, Thomas Krist, and Alan G. Michette. Modern developments in $x$-ray and neutron optics. Springer, 2008. Cited on p. 36

[137] M. Zürch, J. Rothhardt, S. Hädrich, S. Demmler, M. Krebs, J. Limpert, A. Tünnermann, A. Guggenmos, U. Kleineberg, and C. Spielmann. Real-time and Subwavelength Ultrafast Coherent Diffraction Imaging in the Extreme Ultraviolet. Sci. Rep., 4:7356, 2014. Cited on p. 36, 60

[138] X. F. Li, a. Lhuillier, M. Ferray, L. a. Lompré, and G. Mainfray. Multiple-harmonic generation in rare gases at high laser intensity. Phys. Rev. A, 39(11):5751-5761, 1989. Cited on p. 37

[139] Carsten Winterfeldt, Christian Spielmann, and Gustav Gerber. Colloquium: Optimal control of high-harmonic generation. Rev. Mod. Phys., 80(1):117-140, 2008. Cited on p. 37

[140] J.C.H. Spence, U. Weierstall, and M. Howells. Coherence and sampling requirements for diffractive imaging. Ultramicroscopy, 101(2-4):149-152, 2004. Cited on p. 37,60

[141] Bo Chen, Brian Abbey, Ruben Dilanian, Eugeniu Balaur, Grant Van Riessen, Mark Junker, Chanh Q. Tran, Michael W M Jones, Andrew G. Peele, Ian McNulty, David J. Vine, Corey T. Putkunz, Harry M. Quiney, and Keith a. Nugent. Diffraction imaging: The limits of partial coherence. Phys. Rev. B - Condens. Matter Mater. Phys., 86(23), 2012. Cited on p. 37, 60

[142] S. Marchesini. A unified evaluation of iterative projection algorithms for phase retrieval. Rev. Sci. Instrum., 78(1):1-10, 2007. Cited on p. 38

[143] J. R. Fienup, T. R. Crimmins, and W. Holsztynski. Reconstruction of the support of an object from the support of its autocorrelation. J. Opt. Soc. Am., 72(5):610, 1982. Cited on p. 38

[144] David Shapiro, David Shapiro, Pierre Thibault, Pierre Thibault, Tobias Beetz, Tobias Beetz, Veit Elser, Veit Elser, Malcolm Howells, Malcolm Howells, Chris Jacobsen, Chris Jacobsen, Janos Kirz, Janos Kirz, Enju Lima, Enju Lima, Huijie Miao, Huijie Miao, Aaron M Neiman, Aaron M Neiman, David Sayre, and 
David Sayre. Biological imaging by soft x-ray diffraction microscopy. Pnas, 102(43):15343-15346, 2005. Cited on p. 38, 45

[145] H. N. Chapman, A. Barty, S. Marchesini, A. Noy, C. Cui, M. R. Howells, R. Rosen, H. He, J. C. H. Spence, U. Weierstall, T. Beetz, C. Jacobsen, and D. Shapiro. Highresolution ab initio three-dimensional X-ray diffraction microscopy. J. Opt. Soc. Am. A, 23(5):1179 - 1200, 2006. Cited on p. 38

[146] Christian Fuhse and Tim Salditt. Finite-difference field calculations for onedimensionally confined X-ray waveguides. Phys. B Condens. Matter, 357(1-2):5760, 2005. Cited on p. 40, 46

[147] Christian Fuhse and Tim Salditt. Propagation of X-rays in ultra-narrow slits. Opt. Commun., 265(1):140-146, 2006. Cited on p. 40

[148] C. L. Chen. Foundations for guided-wave optics. John Wiley \& Sons, 2006. Cited on p. $41,46,68$

[149] A. V. Martin, F. Wang, N. D. Loh, T. Ekeberg, F. R. N. C. Maia, M. Hantke, G. van der Schot, C. Y. Hampton, R. G. Sierra, A. Aquila, S. Bajt, M. Barthelmess, C. Bostedt, J. D. Bozek, N. Coppola, S. W. Epp, B. Erk, H. Fleckenstein, L. Foucar, M. Frank, H. Graafsma, L. Gumprecht, A. Hartmann, R. Hartmann, G. Hauser, H. Hirsemann, P. Holl, S. Kassemeyer, N. Kimmel, M. Liang, L. Lomb, S. Marchesini, K. Nass, E. Pedersoli, C. Reich, D. Rolles, B. Rudek, A. Rudenko, J. Schulz, R. L. Shoeman, H. Soltau, D. Starodub, J. Steinbrener, F. Stellato, L. Strüder, J. Ullrich, G. Weidenspointner, T. White, C. B. Wunderer, A. Barty, I. Schlichting, M. J. Bogan, and H. N. Chapman. Noise-robust coherent diffractive imaging with a single diffraction pattern. Opt. Express, 20(15):16650, 2012. Cited on p. 43

[150] Klaus Giewekemeyer, Pierre Thibault, Sebastian Kalbfleisch, André Beerlink, Cameron M Kewish, Martin Dierolf, Franz Pfeiffer, and Tim Salditt. Quantitative biological imaging by ptychographic x-ray diffraction microscopy. Proc. Natl. Acad. Sci. U. S. A., 107(2):529-534, 2010. Cited on p. 43

[151] S. Marchesini, H. He, H. N. Chapman, S. P. Hau-Riege, A. Noy, M. R. Howells, U. Weierstall, and J. C. H. Spence. X-ray image reconstruction from a diffraction pattern alone. 11(19):5, 2003. Cited on p. 44 
[152] Xibin Zhou, Robynne Lock, Nick Wagner, Wen Li, Henry C. Kapteyn, and Margaret M. Murnane. Elliptically Polarized High-Order Harmonic Emission from Molecules in Linearly Polarized Laser Fields. Phys. Rev. Lett., 102(7):073902, 2009. Cited on p. $50,56,72$

[153] A. L. Cavalieri, N. Muller, Th. Uphues, V. S. Yakovlev, A. Baltuska, B. Horvath, B. Schmidt, L. Blumel, R. Holzwarth, S. Hendel, M. Drescher, U. Kleineberg, P. M. Echenique, R. Kienberger, F. Krausz, and U. Heinzmann. Attosecond spectroscopy in condensed matter. Nature, 449(7165):1029-1032, oct 2007. Cited on p. 50

[154] Sergey Zayko, Eike Mönnich, Murat Sivis, Dong-Du Mai, Tim Salditt, Sascha Schäfer, and Claus Ropers. Coherent diffractive imaging beyond the projection approximation: waveguiding at extreme ultraviolet wavelengths. Opt. Express, 23(15):19911, 2015. Cited on p. 50, 51, 54

[155] Mike Gruntman. Extreme-ultraviolet radiation filtering by freestanding transmission gratings. Appl. Opt., 34(25):5732, 1995. Cited on p. 50, 54

[156] Tobias Mey, Sergey Zayko, Claus Ropers, Bernd Schäfer, and Klaus Mann. Toroidal grating astigmatism of high-harmonics characterized by EUV Hartmann sensor. Opt. Express, 23(12):15310, 2015. Cited on p. 51, 52

[157] B. E. A. Saleh and M. C. Teich. Fundamentals of Photonics. Wiley Series in Pure and Applied Optics. Wiley, 2007. Cited on p. 53, 55, 68, 69

[158] E. E. Scime, E. H. Anderson, D. J. McComas, and M.L. Schattenburg. Extremeultraviolet polarization and filtering with gold transmission gratings. Appl. Opt., 34(4):648-654, 1995. Cited on p. 54

[159] Chieh Chang and Anne Sakdinawat. Ultra-high aspect ratio high-resolution nanofabrication for hard X-ray diffractive optics. Nat Commun, 5, jun 2014. Cited on p. 56,71

[160] Antoine Camper, Amélie Ferré, Valérie Blanchet, Frédéric Burgy, Dominique Descamps, Stéphane Petit, Thierry Ruchon, and Yann Mairesse. Combined highharmonic interferometries for vectorial spectroscopy. Opt. Lett., 40(22):53875390, nov 2015. Cited on p. 56 
[161] H. M. Quiney, K. A. Nugent, and A. G. Peele. Iterative image reconstruction algorithms using wave-front intensity and phase variation. Opt. Lett., 30(13):16381640, 2005. Cited on p. 60

[162] L. W. Whitehead, G. J. Williams, H. M. Quiney, K. A. Nugent, A. G. Peele, D. Paterson, M. D. de Jonge, and I. McNulty. Fresnel diffractive imaging: Experimental study of coherence and curvature. Phys. Rev. B, 77(10):104112, 2008. Cited on p. 60

[163] Ruben A. Dilanian, Bo Chen, Garth J. Williams, Harry M. Quiney, Keith A. Nugent, Sven Teichmann, Peter Hannaford, Lap V. Dao, and Andrew G. Peele. Diffractive imaging using a polychromatic high-harmonic generation soft-X-ray source. J. Appl. Phys., 106(2), 2009. Cited on p. 60

[164] Stefan Witte, Vasco T Tenner, Daniel We Noom, and Kjeld Se Eikema. Lensless diffractive imaging with ultra-broadband table-top sources: from infrared to extreme-ultraviolet wavelengths. Light Sci. Appl., 3(3):e163, 2014. Cited on p. 60

[165] Tatiana Latychevskaia, Yuriy Chushkin, Federico Zontone, and Hans-werner Fink. Imaging outside the box : Resolution enhancement in X-ray coherent diffraction imaging by extrapolation of diffraction patterns. arXiv, 183102, 2015. Cited on p. 67

[166] Pran Mukherjee, Myung-Gyu Kang, Thomas H. Zurbuchen, L. Jay Guo, and Fred A. Herrero. Fabrication of high aspect ratio Si nanogratings with smooth sidewalls for a deep UV-blocking particle filter. J. Vac. Sci. Technol. B Microelectron. Nanom. Struct., 25(6):2645, 2007. Cited on p. 71

[167] S. J. Goh, H. J. M. Bastiaens, B. Vratzov, Q. Huang, F. Bijkerk, and K. J. Boller. Fabrication and characterization of free-standing, high-line-density transmission gratings for the vacuum UV to soft X-ray range. Opt. Express, 23(4):4421, 2015. Cited on p. 71

[168] Boris Vodungbo, Anna Barszczak Sardinha, Julien Gautier, Guillaume Lambert, Constance Valentin, Magali Lozano, Grégory Iaquaniello, Franck Delmotte, Stéphane Sebban, Jan Lüning, and Philippe Zeitoun. Polarization control of high 
order harmonics in the EUV photon energy range. Opt. Express, 19(5):4346-4356, 2011. Cited on p. 72,86

[169] Jürgen Schmidt, Alexander Guggenmos, Michael Hofstetter, Soo Hoon Chew, and Ulf Kleineberg. Generation of circularly polarized high harmonic radiation using a transmission multilayer quarter waveplate. Opt. Express, 23(26):33564, 2015. Cited on p. 72

[170] G. K. Tadesse, R. Klas, S. Demmler, S. Hädrich, I. Wahyutama, M. Steinert, C. Spielmann, M. Zürch, A. Tünnermann, J. Limpert, and J. Rothhardt. High Speed and High Resolution Table-Top Nanoscale Imaging. arXiv:1605.02909, 2016. Cited on p. 76

[171] Tobias Mey. Radiation characteristics of extreme UV and soft X-ray sources. PhD thesis, Georg-August University, 2015. Cited on p. 78

[172] Dimitar Popmintchev, Franklin Dollar, Christopher Mancuso, Ming-chang Chen, Amelia Hankla, Xiaohui Gao, Bonggu Shim, Alexander L Gaeta, Maryam Tarazkar, Dmitri A Romanov, Robert J Levis, Jim A Gaffney, Mark Foord, Stephen B Libby, Agnieszka Jaron-becker, Andreas Becker, Luis Plaja, Margaret M Murnane, Henry C Kapteyn, and Tenio Popmintchev. Ultraviolet surprise: Efficient soft x-ray high-harmonic generation in multiply ionized plasmas. 2015. Cited on p. 78

[173] Bing Shan and Zenghu Chang. Dramatic extension of the high-order harmonic cutoff by using a long-wavelength driving field. Phys. Rev. A, 65(1):20-23, 2001. Cited on p. 79

[174] E. L. Falcão-Filho, V. M. Gkortsas, A. Gordon, and F. X. Kaertner. Conversion efficiency, scaling and global optimization of high harmonic generation. Inst. Electr. Electron. Eng., pages 8-10, 2009. Cited on p. 79

[175] Vladislav S Yakovlev, Misha Ivanov, and Ferenc Krausz. Enhanced phasematching for generation of soft X-ray harmonics and attosecond pulses in atomic gases. Opt. Express, 15(23):15351-15364, 2007. Cited on p. 79 
[176] T. Auguste, F. Catoire, P. Agostini, L. F. DiMauro, C. C. Chirila, V. S. Yakovlev, and P. Salières. Driving-frequency scaling of high-harmonic quantum paths. New J. Phys., 14(10):103014, 2012. Cited on p. 79

[177] M. V. Frolov, N. L. Manakov, T. S. Sarantseva, M. Yu. Emelin, M.Yu. Ryabikin, and Anthony F. Starace. Analytic Description of the High-Energy Plateau in Harmonic Generation by Atoms: Can the Harmonic Power Increase with Increasing Laser Wavelengths. Phys. Rev. Lett., 102(24):243901, 2009. Cited on p. 79

[178] J. Tate, T. Auguste, H. G. Muller, P. Salières, P. Agostini, and L. F. DiMauro. Scaling of Wave-Packet Dynamics in an Intense Midinfrared Field. Phys. Rev. Lett., 98(1):13901, 2007. Cited on p. 79

[179] M. V. Frolov, N. L. Manakov, Wei-Hao Xiong, Liang-You Peng, J. Burgdörfer, and Anthony F. Starace. Scaling laws for high-order-harmonic generation with midinfrared laser pulses. Phys. Rev. A, 92(2):023409, 2015. Cited on p. 79

[180] Anh-Thu Le, Hui Wei, Cheng Jin, Vu Ngoc Tuoc, Toru Morishita, and C.âD. Lin. Universality of Returning Electron Wave Packet in High-Order Harmonic Generation with Midinfrared Laser Pulses. Phys. Rev. Lett., 113(July):033001, 2014. Cited on p. 79

[181] A. D. Shiner, C. Trallero-Herrero, N. Kajumba, H.-C. Bandulet, D. Comtois, F. Légaré, M. Giguère, J-C. Kieffer, P. B. Corkum, and D. M. Villeneuve. Wavelength scaling of high harmonic generation efficiency. Phys. Rev. Lett., 103(73902), 2009. Cited on p. 79

[182] Chien Jen Lai, Giovanni Cirmi, Kyung Han Hong, Jeffrey Moses, Shu Wei Huang, Eduardo Granados, Phillip Keathley, Siddharth Bhardwaj, and Franz X. Kärtner. Wavelength scaling of high harmonic generation close to the multiphoton ionization regime. Phys. Rev. Lett., 111(7):1-5, 2013. Cited on p. 79

[183] P. Colosimo, G. Doumy, C. I. Blaga, J. Wheeler, C. Hauri, F. Catoire, J. Tate, R. Chirla, a. M. March, G. G. Paulus, H. G. Muller, P. Agostini, and L. F. DiMauro. Scaling strong-field interactions towards the classical limit. Nat. Phys., 4(5):386389, 2008. Cited on p. 79 
[184] M. V. Frolov, N. L. Manakov, Wei-Hao Xiong, Liang-You Peng, J. Burgdörfer, and Anthony F. Starace. Comment on "Universality of Returning Electron Wave Packet in High-Order Harmonic Generation with Midinfrared Laser Pulses". Phys. Rev. Lett., 114(6):069301, 2015. Cited on p. 79

[185] Tenio Popmintchev, Ming-Chang Chen, Alon Bahabad, Michael Gerrity, Pavel Sidorenko, Oren Cohen, Ivan P Christov, Margaret M Murnane, and Henry C Kapteyn. Phase matching of high harmonic generation in the soft and hard Xray regions of the spectrum. Proc. Natl. Acad. Sci. U. S. A., 106(26):10516-10521, 2009. Cited on p. 82

[186] S. Watanabe, K. Kondo, Y. Nabekawa, A. Sagisaka, and Y. Kobayashi. Two-Color Phase Control in Tunneling Ionization and Harmonic Generation by a Strong Laser Field and Its Third Harmonic. Phys. Rev. Lett., 73(20):2692, 1994. Cited on p. 82

[187] I. Jong Kim, Chul Min Kim, Hyung Taek Kim, Gae Hwang Lee, Yong Soo Lee, Ju Yun Park, David Jaeyun Cho, and Chang Hee Nam. Highly efficient highharmonic generation in an orthogonally polarized two-color laser field. Phys. Rev. Lett., 94(24):2-5, 2005. Cited on p. 82

[188] Cheng Jin, Guoli Wang, Hui Wei, Anh-Thu Le, and C. D. Lin. Waveforms for optimal sub-keV high-order harmonics with synthesized two- or three-colour laser fields. Nat. Commun., 5(May):1-6, 2014. Cited on p. 82

[189] Cheng Jin, Gregory J Stein, Kyung-han Hong, and C D Lin. Generation of Bright, Spatially Coherent Soft X-Ray High Harmonics in a Hollow Waveguide Using Two-Color Synthesized Laser Pulses. 043901(July):1-6, 2015. Cited on p. 82

[190] L. E. Chipperfield, J. S. Robinson, J. W. G. Tisch, and J. P. Marangos. Ideal Waveform to Generate the Maximum Possible Electron Recollision Energy for Any Given Oscillation Period. Phys. Rev. Lett., 102(6):063003, 2009. Cited on p. 83

[191] S. Odžak and D. B. Milošević. High-order harmonic generation in the presence of a static electric field. Phys. Rev. A, 72:033407, 2005. Cited on p. 83 
[192] F. A. Weihe, S. K. Dutta, G. Korn, D. Du, P. H. Bucksbaum, and P. L. Shkolnikov. Polarization of high-intensity high-harmonic generation. Phys. Rev. A, 51(5):R3433-R3436, 1995. Cited on p. 83

[193] Ofer Kfir, Patrik Grychtol, Emrah Turgut, Ronny Knut, Dmitriy Zusin, Dimitar Popmintchev, Tenio Popmintchev, Hans Nembach, Justin M. Shaw, Avner Fleischer, Henry Kapteyn, Margaret Murnane, and Oren Cohen. Generation of bright phase-matched circularly-polarized extreme ultraviolet high harmonics. Nat. Photonics, 9(2):99-105, 2014. Cited on p. 84, 85

[194] F. Willems, C. T. L. Smeenk, N. Zhavoronkov, O. Kornilov, I. Radu, M. Schmidbauer, M. Hanke, C. von Korff Schmising, M. J. J. Vrakking, and S. Eisebitt. Probing ultrafast spin dynamics with high-harmonic magnetic circular dichroism spectroscopy. Phys. Rev. B, 92(22):220405, 2015. Cited on p. 86

[195] Ofer Kfir, Eliyahu Bordo, Gil Ilan Haham, Oren Lahav, Avner Fleischer, and Oren Cohen. In-line production of a bi-circular field for generation of helically polarized high-order harmonics. Appl. Phys. Lett., 108(21), 2016. Cited on p. 86, 87

[196] S. Valencia, A. Gaupp, W. Gudat, H. Ch Mertins, P. M. Oppeneer, D. Abramsohn, and C. M. Schneider. Faraday rotation spectra at shallow core levels: $3 p$ edges of Fe, Co, and Ni. New J. Phys., 8, 2006. Cited on p. 87

[197] Thomas A. Duckworth, Feodor Ogrin, Sarnjeet S. Dhesi, Sean Langridge, Amy Whiteside, Thomas Moore, Guillaume Beutier, and Gerrit van der Laan. Magnetic imaging by x-ray holography using extended references. Opt. Express, 19(17):16223-16228, aug 2011. Cited on p. 88

[198] Peter Fischer. X-ray imaging of magnetic structures. IEEE Trans. Magn., 9464(c):1-1, 2014. Cited on p. 88

[199] Stefan Schaffert, Bastian Pfau, Jan Geilhufe, Christian M. Günther, Michael Schneider, Clemens von Korff Schmising, and Stefan Eisebitt. High-resolution magnetic-domain imaging by Fourier transform holography at $21 \mathrm{~nm}$ wavelength. New J. Phys., 15(9):093042, 2013. Cited on p. 88 
[200] S. Kazamias, F. Weihe, D. Douillet, C. Valentin, T. Planchon, S. Sebban, G. Grillon, F. Augé, D. Hulin, and Ph. Balcou. High order harmonic generation optimization with an apertured laser beam. Eur. Phys. J. D, 21(3):353-359, 2002. Cited on p. 91

[201] Hung-Wei Sun, Pei-Chi Huang, Yi-Hsuan Tzeng, Ren-Ting Huang, Ming-Chang Chen, Chii-Dong Lin, and Cheng Jin. Extended Phase Matching of High Harmonic Generation Driven by Truncated Beams in Tight Focusing Geometry. In Conf. Lasers Electro-Optics, page FF2M.5. Optical Society of America, 2016. Cited on p. 91

[202] Hironori Igarashi, Ayumu Makida, Motohiko Ito, and Taro Sekikawa. Pulse compression of phase-matched high harmonic pulses from a time-delay compensated monochromator. Opt. Express, 20(4):3725-32, 2012. Cited on p. 93

[203] Chengyuan Ding, Wei Xiong, Tingting Fan, Daniel Hickstein, Tenio Popmintchev, Xiaoshi Zhang, Mike Walls, Margaret Murnane, and Henry Kapteyn. High flux coherent supercontinuum soft X-ray source driven by a single-stage $10 \mathrm{~mJ}, \mathrm{kHz}$, Ti:sapphire laser amplifier. Cleo 2014, page FTu3B.2, 2014. Cited on p. 93

[204] Motohiko Ito, Yoshimasa Kataoka, Tatsuya Okamoto, Mikio Yamashita, and Taro Sekikawa. Spatiotemporal characterization of single-order high harmonic pulses from time-compensated toroidal-grating monochromator. Opt. Express, 18(6):6071-6078, 2010. Cited on p. 93

[205] Julia Sutherland, E. Christensen, N. Powers, S. Rhynard, J. Painter, and J. Peatross. High harmonic generation in a semi-infinite gas cell. Opt. Express, 12(19):4430 4436, 2004. Cited on p. 97

[206] Georg-August University School of Science (GAUSS) Doctoral Degree Regulations (RerNat-O), 2012. Cited on p. 127 
Appendix A Bibliography 


\section{Author contribution}

This chapter provides information about the particular contributions of the author in the publications that constitute Chapters 2-4.

The following three peer-reviewed articles fulfill the requirements of a cumulative thesis according to the doctoral degree regulations of the $\mathrm{PhD}$ program ProPhys at the GeorgAugust University School of Science (GAUSS) Göttingen [206]:

(1) Tobias Mey, Sergey Zayko, Claus Ropers, Bernd Schäfer, and Klaus Mann

Toroidal grating astigmatism of high-harmonics characterized by EUV Hartmann sensor, Optics Express, 23, 15310-15315 (2015)

(2) Sergey Zayko, Eike Mönnich, Murat Sivis, Dong-Du Mai, Tim Salditt, Sascha Schäfer, and Claus Ropers

Coherent diffractive imaging beyond the projection approximation: Waveguiding at extreme ultraviolet wavelengths, Optics Express, 23, 19911-19921 (2015)

(3) Sergey Zayko, Murat Sivis, Sascha Schäfer, and Claus Ropers

Polarization contrast of nanoscale waveguides in high harmonic imaging, Optica,

Vol. 3, issue 3, pp. 239-242 (2016).

These publications present the original work by the author (S.Z.). Specifically, the contribution of the author concerns the intention, design, source development and conduction of all the experiments presented in Publications (1), (2) and (3), as well as the analysis and interpretation of the collected data. The results were discussed by all authors of the respective publications.

The wavefront measurements described in Chapter 2 were carried out in a collaboration with Tobias Mey, who also implemented a theoretical description for the experimental data and drafted the paper, with contributions and revisions from all authors.

The outcome presented in (2) is resulted from the teamwork of the author with Eike Mönnich, who together with Tim Salditt, developed and modified the phase retrieval algorithms to harness the developed EUV source for CDI. The numerical simulations and 
the analytical solution were conducted by S.Z. and C.R. The EUV source development, experiments and data analysis were carried out by S.Z. Sample preparation and SEM images were provided by M.S. The manuscript was written by S.Z., T.S. and C.R., and revised by all respective authors.

The experiments and data analysis presented in (3) were conducted by S.Z and sample preparation was done by M.S and S.Z. All authors were involved in intensive discussions of the experimental findings at all stages of the study. S.Z. drafted the paper, which was revised by all authors of the respective publication. 


\section{Publications and Conferences}

\section{Publications related to this work}

- Sergey Zayko, Murat Sivis, Sascha Schäfer, and Claus Ropers Polarization contrast of nanoscale waveguides in high harmonic imaging, Optica, 3, issue 3, pp. 239-242 (2016)

- Sergey Zayko, Eike Mönnich, Murat Sivis, Dong-Du Mai, Tim Salditt, Sascha Schäfer, and Claus Ropers Coherent diffractive imaging beyond the projection approximation: Waveguiding at extreme ultraviolet wavelengths, Optics Express, 23, 19911-19921 (2015)

- Tobias Mey, Sergey Zayko, Claus Ropers, Bernd Schäfer, and Klaus Mann Toroidal grating astigmatism of high-harmonics characterized by EUV Hartmann sensor, Optics Express, 23, 15310-15315 (2015).

\section{Conference talks}

- Polarization contrast of nanoscale waveguides studied by coherent diffractive imaging with high-harmonic source, DPG Spring Meeting 2016, Regensburg, Germany

- Waveguiding, mode filtering, and polarization control at extreme-ultraviolet frequencies, SPIE Optics+Photonics 2015, San Diego, USA

- Waveguiding and polarization control of extreme-ultraviolet radiation, DPG Spring Meeting 2015, Heidelberg, Germany

- High resolution coherent diffractive imaging with a table-top high harmonic source, DPG Spring Meeting 2014, Dresden, Germany

- High Harmonic Generation For Coherent Diffractive Imaging, Poster session in DPG Spring Meeting 2013, Regensburg, Germany 



\section{Acknowledgments}

I would like to use this opportunity to thank everyone who supported me throughout the course of this $\mathrm{PhD}$ project.

Foremost I want to express my sincere appreciation to my advisor Prof. Dr. Claus Ropers for giving me the opportunity to carry out my doctoral research in the first place. I feel fortunate to have had Dr. Ropers as my advisor and to have worked in his group among truly talented people. I guess we are all indebted to him for putting together such a great group and for creating a friendly work environment. I thank him for his guidance and support, for all that time and efforts that he has generously invested into my career and personal growths.

I am very thankful to my second advisor Prof. Dr. Tim Salditt. I have benefited from his efforts to make things work in the lab and to extend our capabilities from numerical simulations to the actual experimental data. It was a pleasure working with him and his excitement about my work was always very encouraging and stimulating.

I would like to express my special thanks to my colleague and a friend Dr. Max Gulde. Working closely with such an extraordinary person has been a great privilege and megafun! I cherish every moment spent with him and I am very thankful for all his help and support. I would also like to give my appreciations to other members of the group that were always eager to help and to jointly confront difficulties arising during the work process. I am very thankful to Dr. Murat Sivis who became a mentor and a role model for me, especially in the beginning of my work. I appreciate the fact that I could always appeal to him and get a valuable advice regardless of whether it was a work challenge or a personal question. I express my warm thanks to Dr. Reiner Bormann, Dr. Georg Herink, Dr. Sascha Schäfer and Prof. Dr. Sergey Yalunin and Dr. Ofer Kfir for our numerous discussions about my work and for proofreading my thesis. It was always a pleasure for me spending time with you at university as well as during our free time. Many thanks to Eike Mönnich, Dr. Tobias Mey, Dr. Ofer Kfir and Gerrit Horstmann for the support and a great time during our collaborative efforts in lab hours and a special thanks for proofreading 
my thesis and for the help with graphics. I want to thank Thomas Danz for the pleasant atmosphere in the office and my special thanks are also extended to all the members of the Ropers AG, who to some extent assisted me during my PhD work.

I appreciate all the love and support of my family and friends who have been very encouraging throughout my PhD studies, especially Kristina Saliy for the invaluable help and a nice time in Göttingen. I want to give special thanks to my mom who did all her best to provide me with everything I could have ever needed. Her love and support always kept me motivated, and I will forever be grateful for her help, faith and trust in me.

I gratefully acknowledge the financial support of my project by DFG (SFB755) and Erasmus Mundus partnership within the Triple I consortium for the scholarship which provided me with an opportunity to pursue my $\mathrm{PhD}$ degree in Germany as well as present the results at the international conferences. 